\title{
Asset-based microfinance for microenterprises: Evidence from Pakistan
}

\author{
Appendix for Online Publication \\ Faisal Bari* \\ (r) \\ Kashif Malik $^{\dagger} \quad$ (I) \\ Muhammad Meki ${ }^{\ddagger}$ (r) \\ Simon Quinn ${ }^{\S} \quad$ (r)
}

January 2021

${ }^{*}$ Lahore University of Management Sciences: bari@lums.edu.pk.

${ }^{\dagger}$ Lahore University of Management Sciences: kashif.malik@lums.edu.pk.

$\ddagger$ University of Oxford; Department of International Development and Centre for Islamic Studies: muhammad.meki@qeh.ox.ac.uk.

\$University of Oxford; Department of Economics, St Antony's College and Centre for the Study of African Economies: simon.quinn@economics.ox.ac.uk. 


\section{Contents}

A Further details on contract structure 3

B Elicitation of behavioural characteristics $\quad 4$

$\begin{array}{lll}\text { C Descriptive statistics and randomisation balance } & 6\end{array}$

$\begin{array}{lll}\text { D } & \text { Procedure for assignment to treatment } & 7\end{array}$

$\begin{array}{lll}\text { E } & \text { Characteristics of those who took up the contract } & 7\end{array}$

F Details of assets funded $\quad 9$

G Additional ITT regressions $\quad 11$

$\begin{array}{ll}\text { H LATE estimates for pooled treatments } & 17\end{array}$

I ITT and LATE estimates, separating treatment 1 and treatment 2

$\begin{array}{ll}\text { J Disaggregating results by survey wave } & 26\end{array}$

K Robustness to outliers $\quad 32$

L Local Average Treatment Effect estimations 33

M Heterogeneity analysis 36

M.1 Heterogeneity in take-up . . . . . . . . . . . . . . . 36

M.2 Heterogeneity in usage of the flexible-repayment option . . . . . . . . . 37

M.3 Heterogeneity in outcomes . . . . . . . . . . . . . . . . 38

$\begin{array}{ll}\mathrm{N} \text { Script describing the fixed-repayment contract } & 47\end{array}$

O Further details on the structural model

O.1 Introducing microfinance to the base model . . . . . . . . . . . . 51

O.2 First-stage GMM estimation . . . . . . . . . . . . . . . 51

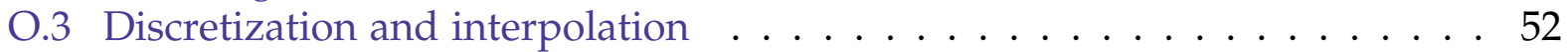

0.4 Simulated moments . . . . . . . . . . . . . . . . . . . 52

0.5 Goodness of fit . . . . . . . . . . . . . . . . 53

0.6 Phase diagram . . . . . . . . . . . . . . . . . . . 58

O.7 Belief-elicitation exercise: depreciation and partial irreversibility parameters 59

O.7.1 Estimation procedure . . . . . . . . . . . . . . 59

O.7.2 Sample and survey . . . . . . . . . . . . . . . . . . 60

0.7 .3 Incentivisation . . . . . . . . . . . . . . . . . . . . . . . . . . . . 61

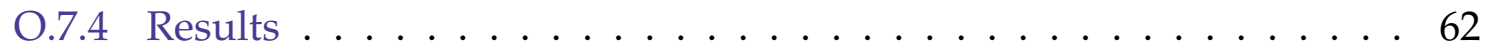




\section{A Further details on contract structure}

In this section, we provide two examples for the payment structure under the flexiblerepayment contract, again using an initial asset value of $\$ 1,000$.

Table A.1: CONTRACT STRUCTURE: FLEXIBLE-REPAYMENT CONTRACT

\begin{tabular}{|c|c|c|c|c|c|c|c|c|}
\hline \multirow{2}{*}{ MONTH } & \multirow{2}{*}{$\begin{array}{c}\text { MFI } \\
\text { OWNERSHIP }\end{array}$} & \multicolumn{3}{|c|}{ PAYMENT EXAMPLE 1} & \multirow{2}{*}{$\begin{array}{c}\text { MFI } \\
\text { OWNERSHIP }\end{array}$} & \multicolumn{3}{|c|}{ PAYMENT EXAMPLE 2} \\
\hline & & RENT & OWNERSHIP & TOTAL & & RENT & OWNERSHIP & TOTAL \\
\hline 1 & $90.0 \%$ & $\overline{99.00}$ & 25.00 & 34.00 & $90 \%$ & 9.00 & 100.00 & 109.00 \\
\hline 2 & $87.5 \%$ & 8.75 & 25.00 & 33.75 & $80 \%$ & 8.00 & 100.00 & 108.00 \\
\hline 3 & $85.0 \%$ & 8.50 & 25.00 & 33.50 & $70 \%$ & 7.00 & 100.00 & 107.00 \\
\hline 4 & $82.0 \%$ & 8.25 & 25.00 & 33.25 & $60 \%$ & 6.00 & 100.00 & 106.00 \\
\hline 5 & $80.0 \%$ & 8.00 & 25.00 & 33.00 & $50 \%$ & 5.00 & 100.00 & 105.00 \\
\hline 6 & $77.5 \%$ & 7.75 & 25.00 & 32.75 & $40 \%$ & 4.00 & 100.00 & 104.00 \\
\hline 7 & $75.0 \%$ & 7.50 & 25.00 & 32.50 & $30 \%$ & 3.00 & 100.00 & 103.00 \\
\hline 8 & $72.5 \%$ & 7.25 & 25.00 & 32.25 & $20 \%$ & 2.00 & 100.00 & 102.00 \\
\hline 9 & $70.0 \%$ & 7.00 & 25.00 & 32.00 & $10 \%$ & 1.00 & 100.00 & 101.00 \\
\hline 10 & $67.5 \%$ & 6.75 & 25.00 & 31.75 & · & · & . & \\
\hline 11 & $65.0 \%$ & 6.50 & 25.00 & 31.50 & . & . & . & . \\
\hline 12 & $62.5 \%$ & 6.25 & 25.00 & 31.25 & . & . & . & . \\
\hline 13 & $60.0 \%$ & 6.00 & 25.00 & 31.00 & . & . & . & . \\
\hline 14 & $57.5 \%$ & 5.75 & 25.00 & 30.75 & . & . & . & . \\
\hline 15 & $55.0 \%$ & 5.50 & 25.00 & 30.50 & . & . & . & . \\
\hline 16 & $52.5 \%$ & 5.25 & 25.00 & 30.25 & . & . & . & . \\
\hline 17 & $50.0 \%$ & 5.00 & 25.00 & 30.00 & . & . & . & . \\
\hline 18 & $47.5 \%$ & 4.75 & 25.00 & 29.75 & . & . & . & . \\
\hline TOTAL & & 123.75 & 450.00 & 573.75 & & 45.00 & 900.00 & 945.00 \\
\hline
\end{tabular}

Note: This table provides an example of the required payment structure under the flexible-repayment contract for an asset costing $\$ 1,000$, where the client has paid $\$ 100$ to initially purchase $10 \%$ of the asset. A nominal annual rental rate of $12 \%$ implies monthly rent of $1 \%$ of the asset's value, which is $\$ 100$. In addition to the rent, the client is also obliged to purchase $2.5 \%$ of the MFI's ownership share each month, based on the initial asset value of $\$ 1,000$, which implies an amount of $\$ 25$. The two example provide different potential repayment schedules, based on the client (i) paying the absolute minimum; (ii) paying more and ending the contract early.

The first example illustrates the absolute minimum repayment requirement for the client, which is $\$ 25$ per month. Since the MFI's ownership share decreases more gradually than it does under the fixed-repayment contract, the cumulative rental payments are higher than under the comparable fixed-repayment contract. The second example presents a case where the client repays more than required every month (\$100), which results in a more rapidly decreasing ownership share for the MFI (and lower rental payments), and the contract ending at the end of the ninth month.

Both contracts were designed to be consistent with locally accepted financial norms. In modern legal terms, it resembles a 'hire-purchase' contract, which shares features with both 'rent-to-own' structures (a more commonly used term in the United States) as well as lease agreements. The exact difference between these terms is less rele- 
vant in our context, given the informal nature of most microenterprises, which are often not registered for taxes and do not file standardised accounts. As an example of the accounting and tax implications of different contractual features for formal firms, in an 'operating lease' the monthly payment is equivalent to rent and treated as a standard business expense; in contrast, a 'financial lease', which contains an option for ownership transfer of the asset, is treated like a loan and the lessee can reduce their taxable income by claiming both interest rate and depreciation expenses. For details of the nuanced difference between hire-purchase and rent-to-own agreements, see https://www.investopedia.com/terms/h/hire-purchase.asp.

\section{B Elicitation of behavioural characteristics}

In this section, we describe how we implemented behavioural games to measure individual preferences. Before conducting all activities, participants were informed that, at the end of the behavioural games session, one of the incentivised activities would be selected for payment by physically drawing a ball from a bag. Within the selected activity, balls would be drawn to select the one final question that would be used for payment. As such, participants were required to answer all questions attentively, because any question could have been selected. This method also allowed the use of payment amounts that were relatively large, with the average payment being three times as large as the median daily business profits for microenterprises in the sample. ${ }^{1}$

The incentivised measure of risk preferences is based on a certainty equivalent elicitation procedure that involved a series of 30 questions requiring respondents to choose between a guaranteed amount of money or an uncertain investment option, which had two possible outcomes: (i) a 'bad' outcome, with a payoff of zero; or (ii) a 'good' outcome, with a payoff of PKR $1,000 .^{2}$

In the loss aversion elicitation exercise, respondents were offered a series of binaryoutcome investment choices that involved a large positive outcome or a (gradually increasing) negative outcome, which they could accept or reject. If they accepted the investments and the loss aversion activity was chosen for payment at the end of the workshop, then a realised loss would be taken out of their guaranteed workshop participation fee; as such, this represented a potential real loss. ${ }^{3}$

In the time preference elicitation activity, individuals were offered a series of choices between an amount of money paid on the same day as the workshop or (gradually in-

\footnotetext{
${ }^{1}$ From a methodological perspective, Charness, Gneezy, and Halladay (2016) show that paying for only a (randomly selected) subset of all activities is at least as effective as paying for all of them, and can actually be more effective in terms of helping to avoid wealth effects and hedging within the behavioural games session.

2 We adapted the measures used by Barr and Packard (2002) and Vieider, Lefebvre, Bouchouicha, Chmura, Hakimov, Krawczyk, and Martinsson (2015).

${ }^{3}$ We adapted the loss aversion measure used by Bartling, Fehr, and Herz (2014).
} 
creasing) amounts of money one month from the workshop. The time-preference activity was also conducted using a 'far frame', where money was offered one month forward versus two months forward.

For all incentivised measures, a simple (unweighted) index was created for the number of decisions made (for example, the number of rejections of the risky investments, indicating risk aversion or loss aversion, or the number of rejections of future payment amounts, indicating a preference for money today). This index was then split into equalsized terciles (as close as possible), based on the recommendations of Gelman and Park (2009). 


\section{Descriptive statistics and randomisation balance}

Table A.2: Summary statistics

\begin{tabular}{|c|c|c|c|c|c|c|c|}
\hline Variable & $\begin{array}{c}(1) \\
\text { Control } \\
\text { Mean/SE }\end{array}$ & $\begin{array}{c}(2) \\
\text { Treatment: fixed } \\
\text { Mean/SE }\end{array}$ & $\begin{array}{c}(3) \\
\text { Treatment: flexible } \\
\text { Mean/SE }\end{array}$ & $\begin{array}{c}(4) \\
\text { Total } \\
\text { Mean/SE }\end{array}$ & $(1)-(2)$ & $\begin{array}{c}\text { Normalized } \\
\text { difference } \\
(1)-(3)\end{array}$ & (2)-(3) \\
\hline Female & $\begin{array}{l}0.06 \\
(0.01)\end{array}$ & $\begin{array}{c}0.10 \\
(0.02)\end{array}$ & $\begin{array}{c}0.09 \\
(0.02)\end{array}$ & $\begin{array}{c}0.08 \\
(0.01)\end{array}$ & -0.15 & -0.13 & 0.03 \\
\hline Age & $\begin{array}{l}37.10 \\
(0.65)\end{array}$ & $\begin{array}{l}37.97 \\
(0.61)\end{array}$ & $\begin{array}{l}38.65 \\
(0.67)\end{array}$ & $\begin{array}{l}37.90 \\
(0.37)\end{array}$ & -0.09 & -0.15 & -0.07 \\
\hline Household size & $\begin{array}{c}6.11 \\
(0.15)\end{array}$ & $\begin{array}{c}6.35 \\
(0.21)\end{array}$ & $\begin{array}{c}6.49 \\
(0.16)\end{array}$ & $\begin{array}{c}6.31 \\
(0.10)\end{array}$ & -0.08 & -0.16 & -0.05 \\
\hline Household earners & $\begin{array}{c}1.91 \\
(0.07)\end{array}$ & $\begin{array}{l}1.93 \\
(0.08)\end{array}$ & $\begin{array}{c}2.05 \\
(0.07)\end{array}$ & $\begin{array}{l}1.96 \\
(0.04)\end{array}$ & -0.02 & -0.14 & -0.10 \\
\hline Distance to MFI office (minutes) & $\begin{array}{l}16.14 \\
(1.28)\end{array}$ & $\begin{array}{l}15.00 \\
(0.91)\end{array}$ & $\begin{array}{l}14.72 \\
(1.00)\end{array}$ & $\begin{array}{l}15.29 \\
(0.62)\end{array}$ & 0.06 & 0.08 & 0.02 \\
\hline Runs a rickshaw business & $\begin{array}{l}0.20 \\
(0.03)\end{array}$ & $\begin{array}{c}0.19 \\
(0.02)\end{array}$ & $\begin{array}{l}0.20 \\
(0.03)\end{array}$ & $\begin{array}{c}0.19 \\
(0.01)\end{array}$ & 0.04 & 0.01 & -0.02 \\
\hline Business experience (years) & $\begin{array}{l}9.31 \\
(0.49)\end{array}$ & $\begin{array}{l}9.75 \\
(0.47)\end{array}$ & $\begin{array}{l}9.78 \\
(0.56)\end{array}$ & $\begin{array}{l}9.61 \\
(0.29)\end{array}$ & -0.06 & -0.06 & -0.00 \\
\hline Number of businesses managed & $\begin{array}{c}1.22 \\
(0.03)\end{array}$ & $\begin{array}{c}1.19 \\
(0.03)\end{array}$ & $\begin{array}{l}1.25 \\
(0.03)\end{array}$ & $\begin{array}{l}1.22 \\
(0.02)\end{array}$ & 0.06 & -0.05 & -0.11 \\
\hline Business revenue & $\begin{array}{l}728.19 \\
(49.10)\end{array}$ & $\begin{array}{l}734.06 \\
(46.93)\end{array}$ & $\begin{array}{l}721.17 \\
(49.31)\end{array}$ & $\begin{array}{l}727.90 \\
(27.93)\end{array}$ & -0.01 & 0.01 & 0.02 \\
\hline Business profits & $\begin{array}{l}234.95 \\
(9.97)\end{array}$ & $\begin{array}{l}252.10 \\
(10.52)\end{array}$ & $\begin{array}{c}249.48 \\
(9.79)\end{array}$ & $\begin{array}{l}245.49 \\
(5.84)\end{array}$ & -0.10 & -0.09 & 0.02 \\
\hline Number of employees & $\begin{array}{c}0.94 \\
(0.11)\end{array}$ & $\begin{array}{l}0.85 \\
(0.09)\end{array}$ & $\begin{array}{l}1.00 \\
(0.10)\end{array}$ & $\begin{array}{c}0.93 \\
(0.06)\end{array}$ & 0.06 & -0.03 & -0.10 \\
\hline Total fixed assets & $\begin{array}{l}851.44 \\
(95.51)\end{array}$ & $\begin{array}{l}943.67 \\
(97.25)\end{array}$ & $\begin{array}{c}967.15 \\
(108.05)\end{array}$ & $\begin{array}{l}920.35 \\
(57.83)\end{array}$ & -0.06 & -0.07 & -0.01 \\
\hline Current assets: cash & $\begin{array}{l}175.79 \\
(16.40)\end{array}$ & $\begin{array}{l}193.10 \\
(17.70)\end{array}$ & $\begin{array}{l}197.03 \\
(18.46)\end{array}$ & $\begin{array}{l}188.57 \\
(10.11)\end{array}$ & -0.06 & -0.08 & -0.01 \\
\hline Current assets: debt & $\begin{array}{l}137.10 \\
(24.27)\end{array}$ & $\begin{array}{l}116.21 \\
(20.66)\end{array}$ & $\begin{array}{l}128.07 \\
(25.14)\end{array}$ & $\begin{array}{l}127.07 \\
(13.49)\end{array}$ & 0.06 & 0.02 & -0.03 \\
\hline Current assets: inventories & $\begin{array}{l}320.43 \\
(40.51)\end{array}$ & $\begin{array}{l}320.00 \\
(37.98)\end{array}$ & $\begin{array}{l}311.10 \\
(35.86)\end{array}$ & $\begin{array}{l}317.25 \\
(22.03)\end{array}$ & 0.00 & 0.02 & 0.02 \\
\hline Wage income & $\begin{array}{l}21.74 \\
(3.97)\end{array}$ & $\begin{array}{l}25.97 \\
(4.37)\end{array}$ & $\begin{array}{l}27.53 \\
(4.21)\end{array}$ & $\begin{array}{l}25.06 \\
(2.42)\end{array}$ & -0.06 & -0.09 & -0.02 \\
\hline Total household income & $\begin{array}{l}345.29 \\
(13.42)\end{array}$ & $\begin{array}{l}349.23 \\
(13.36)\end{array}$ & $\begin{array}{l}367.38 \\
(13.46)\end{array}$ & $\begin{array}{l}353.80 \\
(7.74)\end{array}$ & -0.02 & -0.10 & -0.09 \\
\hline Household consumption expenditure & $\begin{array}{c}200.69 \\
(7.01)\end{array}$ & $\begin{array}{c}219.79 \\
(7.85)\end{array}$ & $\begin{array}{c}213.75 \\
(7.50)\end{array}$ & $\begin{array}{l}211.42 \\
(4.31)\end{array}$ & -0.16 & -0.11 & 0.05 \\
\hline Household savings & $\begin{array}{l}432.10 \\
(58.05)\end{array}$ & $\begin{array}{l}426.05 \\
(50.46)\end{array}$ & $\begin{array}{l}470.13 \\
(61.16)\end{array}$ & $\begin{array}{l}442.40 \\
(32.64)\end{array}$ & 0.01 & -0.04 & -0.05 \\
\hline Household loans & $\begin{array}{l}37.80 \\
(4.65)\end{array}$ & $\begin{array}{l}34.50 \\
(4.65)\end{array}$ & $\begin{array}{l}43.53 \\
(5.15)\end{array}$ & $\begin{array}{l}38.54 \\
(2.78)\end{array}$ & 0.04 & -0.07 & -0.12 \\
\hline Management practices index & $\begin{array}{l}0.00 \\
(0.04)\end{array}$ & $\begin{array}{l}0.03 \\
(0.05)\end{array}$ & $\begin{array}{l}0.05 \\
(0.05)\end{array}$ & $\begin{array}{c}0.02 \\
(0.03)\end{array}$ & -0.04 & -0.06 & -0.02 \\
\hline Risk aversion index & $\begin{array}{l}21.10 \\
(0.57)\end{array}$ & $\begin{array}{l}21.88 \\
(0.58)\end{array}$ & $\begin{array}{l}21.93 \\
(0.58)\end{array}$ & $\begin{array}{l}21.63 \\
(0.33)\end{array}$ & -0.09 & -0.09 & -0.01 \\
\hline Loss aversion index & $\begin{array}{l}5.88 \\
(0.16)\end{array}$ & $\begin{array}{l}6.26 \\
(0.17)\end{array}$ & $\begin{array}{l}5.95 \\
(0.17)\end{array}$ & $\begin{array}{l}6.03 \\
(0.10)\end{array}$ & -0.15 & -0.03 & 0.12 \\
\hline Math score index & $\begin{array}{c}0.00 \\
(0.04)\end{array}$ & $\begin{array}{l}-0.03 \\
(0.04)\end{array}$ & $\begin{array}{c}0.01 \\
(0.04)\end{array}$ & $\begin{array}{l}-0.01 \\
(0.02)\end{array}$ & 0.05 & -0.01 & -0.06 \\
\hline Education (years) & $\begin{array}{l}7.64 \\
(0.23)\end{array}$ & $\begin{array}{l}7.08 \\
(0.23)\end{array}$ & $\begin{array}{l}7.69 \\
(0.23)\end{array}$ & $\begin{array}{l}7.46 \\
(0.13)\end{array}$ & 0.15 & -0.01 & -0.16 \\
\hline $\mathrm{N}$ & 254 & 257 & 246 & 757 & & & \\
\hline
\end{tabular}




\section{Procedure for assignment to treatment}

Following the collection of workshop data, and before the visits were conducted, all clients were randomised into three different groups: (i) a control group, who had access to the interest-free loan of $\$ 475$; (ii) a group that were only offered the fixed-repayment contract; and (iii) a group that were offered the flexible-repayment contract, which would subsequently be explained to them. Randomisation was stratified on microenterprise type, performance and gender, using matched sextuplets:

(i) First, the sample was split into three groups: (a) rickshaw drivers (this was the most popular business sector at baseline, at around 20\%); (b) males in all non-rickshaw sectors; and (c) females in non-rickshaw sectors;

(ii) Within each of the three groups, individuals were ordered by the three-month average of their business profits, as collected in the survey;

(iii) Groups of matched sextuplets were then formed, with two individuals being randomly allocated into the three treatment groups in each sextuplet.

Forming matched sextuplets is consistent with the recommendation by Athey and Imbens (2017), who suggest stratifying as much as possible so that each stratum contains at least two treated and two control units. They argue that although using paired designs has some benefits in terms of expected precision, these tend to be small, and do not outweigh the significant costs.

\section{E Characteristics of those who took up the contract}

In this section, we presents the characteristics of those who took up either of our asset finance contracts, compared to those who were assigned to a treatment contract but did not take up the product. There is no difference in characteristics of takers and non-takers in terms of gender, age, household size, number of household earners, the number of businesses in the household or whether they run a rickshaw business (the most popular sector in our data). Those who took up appear to run slightly younger businesses. Perhaps unsurprisingly - given the relatively large deposit required to purchase the initial 10\% of the asset (approximately $\$ 150$ on average) - we find that contract takers have slightly larger and more profitable businesses: higher revenues, profits and current assets in the form of cash, with all differences statistically significant as per the $t$-test reported in the table. They also appear to come from slightly wealthier households in terms of net household assets and monthly consumption expenditure. 
Table A.3: Characteristics of those who took up the asset finance contract

\begin{tabular}{|c|c|c|c|c|c|}
\hline Variable & $\begin{array}{c}(1) \\
\text { Take-up }=0 \\
\text { Mean/SE }\end{array}$ & $\begin{array}{c}(2) \\
\text { Take-up = } 1 \\
\text { Mean/SE }\end{array}$ & $\begin{array}{c}(3) \\
\text { Overall } \\
\text { Mean/SE }\end{array}$ & $\begin{array}{c}\text { T-test } \\
\text { Difference } \\
(1)-(2) \\
\end{array}$ & $\begin{array}{c}\text { Normalized } \\
\text { difference } \\
(1)-(2)\end{array}$ \\
\hline Female & $\begin{array}{c}0.12 \\
(0.02)\end{array}$ & $\begin{array}{c}0.08 \\
(0.02)\end{array}$ & $\begin{array}{c}0.10 \\
(0.01)\end{array}$ & 0.04 & 0.12 \\
\hline Age & $\begin{array}{l}38.81 \\
(0.64)\end{array}$ & $\begin{array}{l}37.90 \\
(0.63)\end{array}$ & $\begin{array}{l}38.30 \\
(0.45)\end{array}$ & 0.91 & 0.09 \\
\hline Household size & $\begin{array}{c}6.35 \\
(0.23)\end{array}$ & $\begin{array}{c}6.47 \\
(0.15)\end{array}$ & $\begin{array}{c}6.42 \\
(0.13)\end{array}$ & -0.13 & -0.04 \\
\hline Household earners & $\begin{array}{c}1.92 \\
(0.08)\end{array}$ & $\begin{array}{c}2.04 \\
(0.07)\end{array}$ & $\begin{array}{c}1.99 \\
(0.05)\end{array}$ & -0.12 & -0.10 \\
\hline Runs a rickshaw business & $\begin{array}{c}0.20 \\
(0.03)\end{array}$ & $\begin{array}{c}0.18 \\
(0.02)\end{array}$ & $\begin{array}{c}0.19 \\
(0.02)\end{array}$ & 0.02 & 0.05 \\
\hline Number of businesses managed & $\begin{array}{c}1.22 \\
(0.03)\end{array}$ & $\begin{array}{c}1.22 \\
(0.03)\end{array}$ & $\begin{array}{c}1.22 \\
(0.02)\end{array}$ & -0.00 & -0.01 \\
\hline Business age (years) & $\begin{array}{l}10.64 \\
(0.58)\end{array}$ & $\begin{array}{c}9.07 \\
(0.46)\end{array}$ & $\begin{array}{c}9.76 \\
(0.36)\end{array}$ & $1.56^{*}$ & 0.19 \\
\hline Business revenue & $\begin{array}{l}706.09 \\
(49.47)\end{array}$ & $\begin{array}{l}744.87 \\
(46.65)\end{array}$ & $\begin{array}{l}727.75 \\
(33.97)\end{array}$ & $-38.78^{*}$ & -0.05 \\
\hline Business profits & $\begin{array}{l}227.65 \\
(9.80)\end{array}$ & $\begin{array}{l}269.11 \\
(10.17)\end{array}$ & $\begin{array}{l}250.82 \\
(7.19)\end{array}$ & $-41.46^{* *}$ & -0.26 \\
\hline Total fixed assets & $\begin{array}{l}789.16 \\
(98.33)\end{array}$ & $\begin{array}{l}1,086.29 \\
(103.36)\end{array}$ & $\begin{array}{l}955.15 \\
(72.46)\end{array}$ & $-297.13^{*}$ & -0.18 \\
\hline Current assets: cash & $\begin{array}{l}163.65 \\
(18.09)\end{array}$ & $\begin{array}{l}219.81 \\
(17.72)\end{array}$ & $\begin{array}{l}195.02 \\
(12.77)\end{array}$ & $-56.16^{*}$ & -0.20 \\
\hline Current assets: debt & $\begin{array}{c}91.55 \\
(18.77)\end{array}$ & $\begin{array}{l}146.08 \\
(24.83)\end{array}$ & $\begin{array}{l}122.01 \\
(16.19)\end{array}$ & -54.53 & -0.15 \\
\hline Current assets: inventories & $\begin{array}{l}315.26 \\
(39.87)\end{array}$ & $\begin{array}{l}315.95 \\
(34.64)\end{array}$ & $\begin{array}{l}315.65 \\
(26.13)\end{array}$ & -0.69 & -0.00 \\
\hline Number of employees & $\begin{array}{c}0.88 \\
(0.10)\end{array}$ & $\begin{array}{c}0.95 \\
(0.09)\end{array}$ & $\begin{array}{c}0.92 \\
(0.07)\end{array}$ & -0.08 & -0.05 \\
\hline Wage income & $\begin{array}{l}23.75 \\
(4.18)\end{array}$ & $\begin{array}{l}29.10 \\
(4.31)\end{array}$ & $\begin{array}{l}26.73 \\
(3.03)\end{array}$ & -5.35 & -0.08 \\
\hline Total household income & $\begin{array}{l}327.35 \\
(13.11)\end{array}$ & $\begin{array}{l}382.40 \\
(13.28)\end{array}$ & $\begin{array}{l}358.10 \\
(9.48)\end{array}$ & -55.05 & -0.26 \\
\hline Household consumption expenditure & $\begin{array}{l}203.13 \\
(7.62)\end{array}$ & $\begin{array}{l}227.66 \\
(7.58)\end{array}$ & $\begin{array}{l}216.84 \\
(5.43)\end{array}$ & $-24.53^{*}$ & -0.20 \\
\hline Household savings & $\begin{array}{l}308.61 \\
(49.07)\end{array}$ & $\begin{array}{l}557.42 \\
(58.30)\end{array}$ & $\begin{array}{l}447.61 \\
(39.46)\end{array}$ & $-248.80^{*}$ & -0.28 \\
\hline Household loans & $\begin{array}{l}55.98 \\
(6.30)\end{array}$ & $\begin{array}{l}25.44 \\
(3.50)\end{array}$ & $\begin{array}{l}38.92 \\
(3.46)\end{array}$ & $30.54^{* * *}$ & 0.39 \\
\hline Math score (above median) & $\begin{array}{c}0.55 \\
(0.03)\end{array}$ & $\begin{array}{c}0.53 \\
(0.03)\end{array}$ & $\begin{array}{c}0.53 \\
(0.02)\end{array}$ & 0.02 & 0.04 \\
\hline Management practices (above median) & $\begin{array}{c}0.50 \\
(0.03)\end{array}$ & $\begin{array}{c}0.51 \\
(0.03)\end{array}$ & $\begin{array}{c}0.50 \\
(0.02)\end{array}$ & -0.01 & -0.03 \\
\hline Risk aversion (above median) & $\begin{array}{c}0.50 \\
(0.03)\end{array}$ & $\begin{array}{c}0.51 \\
(0.03)\end{array}$ & $\begin{array}{c}0.51 \\
(0.02)\end{array}$ & -0.01 & -0.02 \\
\hline Loss aversion (above median) & $\begin{array}{c}0.57 \\
(0.03)\end{array}$ & $\begin{array}{c}0.59 \\
(0.03)\end{array}$ & $\begin{array}{c}0.58 \\
(0.02)\end{array}$ & -0.02 & -0.05 \\
\hline Time preference: impatience (above median) & $\begin{array}{c}0.52 \\
(0.03)\end{array}$ & $\begin{array}{c}0.48 \\
(0.03)\end{array}$ & $\begin{array}{c}0.50 \\
(0.02)\end{array}$ & 0.03 & 0.07 \\
\hline Observations & 222 & 281 & 503 & & \\
\hline
\end{tabular}

Notes: Standard errors are clustered at the individual level. All flow variables are for the last month, and all currency values are in US\$ equivalent based on the prevailing exchange rate during implementation of the projects (USDPKR of approximately 105). Normalized differences are computed as the difference in means divided by the square root of half of the sum of the variances. ${ }^{*} \mathrm{p}<0.10$, ${ }^{* *} \mathrm{p}<0.05$, ${ }^{* * *} \mathrm{p}<0.01$. 


\section{F Details of assets funded}

In the figures below, we illustrate: (i) the different categories of assets chosen by microenterprise owners to be financed under our contracts; (ii) the distribution in the values of those assets.

Figure A.1: Types of asset funded

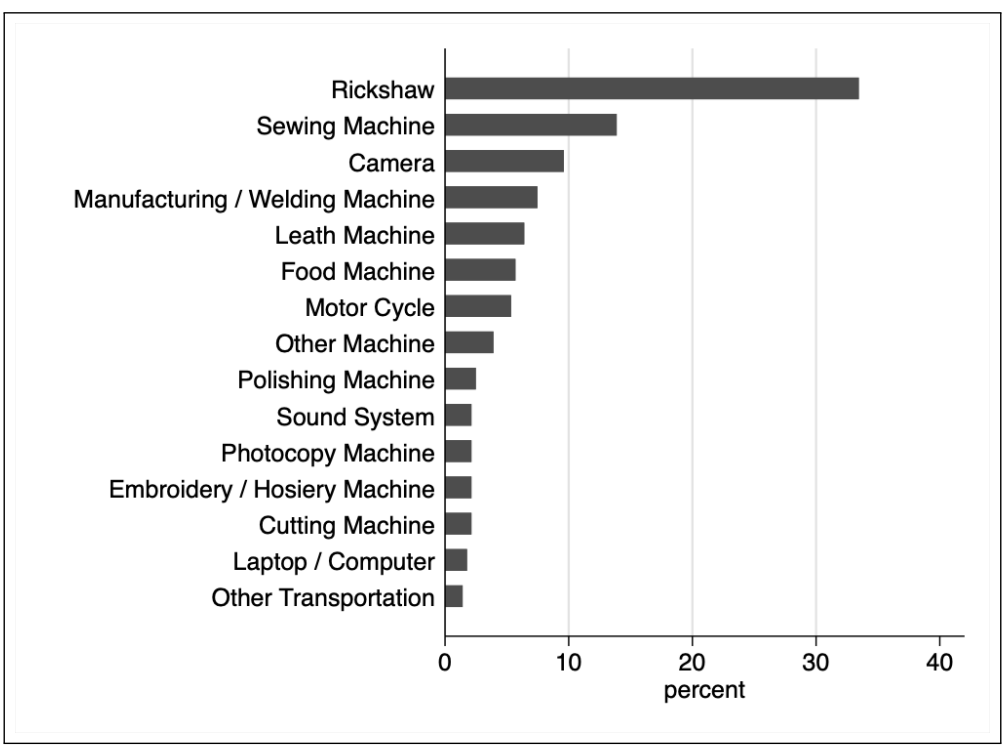

Note: This figure illustrates the different categories of asset chosen by the 281 clients who accepted a treatment contract.

Figure A.2: Distribution of funded asset values

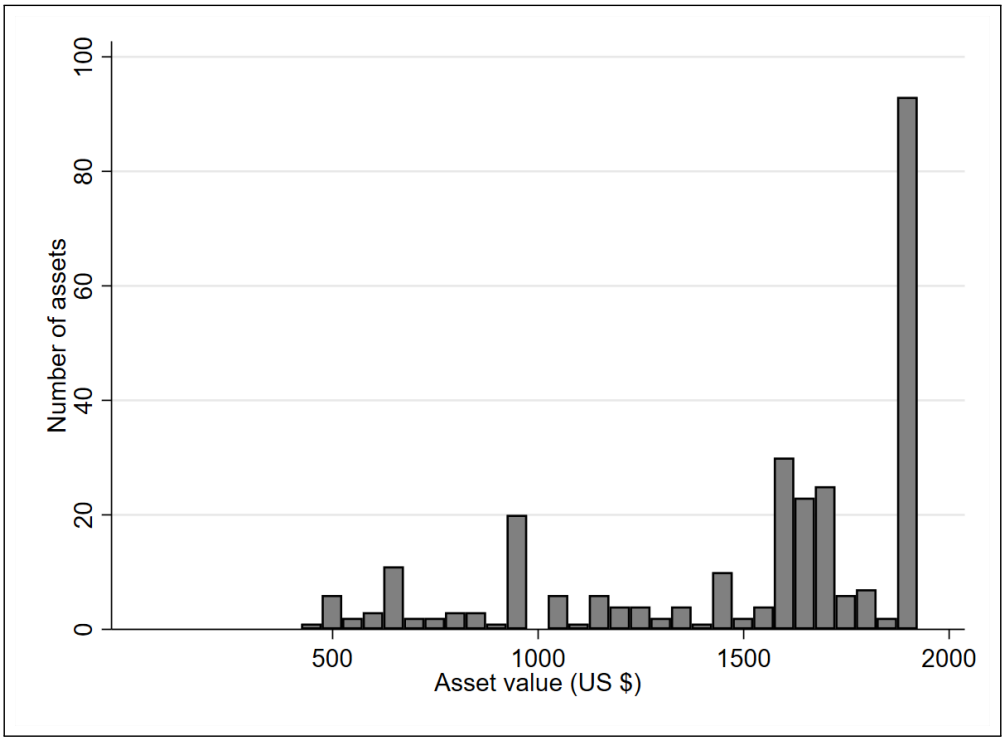

Note: This figure illustrates the distribution in the value of assets financed for clients who took up one of our treatment contracts. Microenterprise owners were permitted to purchase an asset worth up to $\$ 1,900$. 
Below we present results from regressions that investigate the relationship between contract assignment and the value and type of asset chosen by microenterprise owners. The average value of asset financed for those assigned to the fixed-repayment contract was higher than the value for those assigned to the flexible-repayment contract, but the difference is not significant when controlling for stratification dummies (column 1 in the table; $p$-value $=0.233$ ). Column 2 provides some suggestive evidence of more risk-averse individuals choosing higher asset values when offered the flexible contract. The remaining columns show that - for the five most popular assets - there is no clear difference by treatment assignment in the proportion of microenterprise owners choosing that asset.

\begin{tabular}{|c|c|c|c|c|c|c|c|}
\hline & $\begin{array}{c}(1) \\
\text { Asset } \\
\text { Value }\end{array}$ & $\begin{array}{l}(2) \\
\text { Asset } \\
\text { Value }\end{array}$ & Rickshaw & $\begin{array}{c}(4) \\
\text { Sewing } \\
\text { machine }\end{array}$ & Camera & $\begin{array}{c}\text { (6) } \\
\text { Manufacturing } \\
\text { / welding } \\
\text { machine }\end{array}$ & $\begin{array}{c}\text { (7) } \\
\text { Lathe } \\
\text { machine }\end{array}$ \\
\hline Assignment 2 & $\begin{array}{c}59.80 \\
(50.09)\end{array}$ & & $\begin{array}{l}-0.00 \\
(0.03)\end{array}$ & $\begin{array}{l}-0.00 \\
(0.04)\end{array}$ & $\begin{array}{l}0.06^{*} \\
(0.03)\end{array}$ & $\begin{array}{l}-0.00 \\
(0.03)\end{array}$ & $\begin{array}{l}-0.01 \\
(0.03)\end{array}$ \\
\hline Assignment $1 *$ Medium risk aversion & & $\begin{array}{l}-52.64 \\
(90.12)\end{array}$ & & & & & \\
\hline Assignment $1 *$ High risk aversion & & $\begin{array}{c}27.71 \\
(89.29)\end{array}$ & & & & & \\
\hline Assignment $2 *$ Low risk aversion & & $\begin{array}{l}-53.19 \\
(96.23)\end{array}$ & & & & & \\
\hline Assignment $2 *$ Medium risk aversion & & $\begin{array}{c}159.23^{* *} \\
(79.00)\end{array}$ & & & & & \\
\hline Assignment $2 *$ High risk aversion & & $\begin{array}{c}72.79 \\
(85.50)\end{array}$ & & & & & \\
\hline Assignment 1 mean & 1471 & & 0.17 & 0.09 & 0.03 & 0.04 & 0.04 \\
\hline Test: Assignment 1 equal & & 0.381 & & & & & \\
\hline Test: Assignment 2 equal & & 0.051 & & & & & \\
\hline Test: Tercile 2 equal & & 0.008 & & & & & \\
\hline Test: Tercile 3 equal & & 0.609 & & & & & \\
\hline Observations & 281 & 281 & 281 & 281 & 281 & 281 & 281 \\
\hline
\end{tabular}

Note: In column 2, we interact assignment with each of the three baseline risk terciles (where low, medium and high risk aversion refers to individuals who were in the bottom, middle and highest terciles of measured risk aversion using the baseline risk preference elicitation task). We denote significance using $*$ for $10 \%, * *$ for $5 \%$ and $* * *$ for $1 \%$ 


\section{G Additional ITT regressions}

Here we report the effect of treatment on wage employment (extensive and intensive margin).

Table A.4: Treatment effects: Wage work

\begin{tabular}{lcccc}
\hline & $\begin{array}{c}(1) \\
\text { Has a } \\
\text { wage job }\end{array}$ & $\begin{array}{c}\text { Number of } \\
\text { wage jobs }\end{array}$ & $\begin{array}{c}(3) \\
\text { Total } \\
\text { wage hours }\end{array}$ & $\begin{array}{c}(4) \\
\text { Total } \\
\text { wage income }\end{array}$ \\
\hline Assignment & -0.07 & -0.07 & -3.62 & -15.27 \\
& $(0.03)$ & $(0.03)$ & $(1.31)$ & $(6.00)$ \\
& {$[0.01]^{* * *}$} & {$[0.01]^{* *}$} & {$[0.01]^{* * *}$} & {$[0.01]^{* *}$} \\
& $\{0.01\}^{* *}$ & $\{0.01\}^{* *}$ & $\{0.01\}^{* *}$ & $\{0.01\}^{* *}$ \\
\hline Control mean (follow-up) & 0.25 & 0.25 & 12.48 & 55.38 \\
Observations & 3,608 & 3,608 & 3,608 & 3,608 \\
\hline
\end{tabular}

Note: In this table we report the intent-to-treat estimates of the combined treatment on primary outcomes, obtained by least-squares estimation. Below each coefficient, we report a standard error in parenthesis, a $p$-value in brackets, and a $q$-value in curly braces. Standard errors allow for clustering at the level of the individual. $q$-values are obtained using the sharpened procedure of (Benjamini et al., 2006). We denote significance using $*$ for $10 \%, * *$ for $5 \%$ and $* * *$ for $1 \%$.

Here we report the effect of treatment on business cost categories.

Table A.5: Treatment effects: Business costs

\begin{tabular}{|c|c|c|c|c|c|c|c|c|c|}
\hline & $\begin{array}{c}(1) \\
\text { Raw } \\
\text { materials }\end{array}$ & $\begin{array}{c}(2) \\
\text { Wages }\end{array}$ & $\begin{array}{c}\text { (3) } \\
\text { Utility } \\
\text { bills }\end{array}$ & $\begin{array}{c}(4) \\
\text { Rent: } \\
\text { land }\end{array}$ & $\begin{array}{c}\text { (5) } \\
\text { Transport }\end{array}$ & $\begin{array}{c}(6) \\
\text { Rent: } \\
\text { machines }\end{array}$ & $\begin{array}{c}(7) \\
\text { Repairs }\end{array}$ & $\begin{array}{c}(8) \\
\text { Phone }\end{array}$ & $\begin{array}{c}(9) \\
\text { Loan } \\
\text { repayment }\end{array}$ \\
\hline Assignment & $\begin{array}{c}-45.92 \\
(27.60) \\
{[0.10]^{*}} \\
\{0.11\}\end{array}$ & $\begin{array}{c}4.00 \\
(6.18) \\
{[0.52]} \\
\{0.35\} \\
\end{array}$ & $\begin{array}{l}8.11 \\
(1.93) \\
{[0.00]^{* * *}} \\
\{0.00\}^{* * *}\end{array}$ & $\begin{array}{c}2.40 \\
(2.06) \\
{[0.24]} \\
\{0.21\}\end{array}$ & $\begin{array}{l}-0.23 \\
(0.89) \\
{[0.80]} \\
\{0.37\}\end{array}$ & $\begin{array}{l}-3.13 \\
(0.93) \\
{[0.00]^{* * *}} \\
\{0.00\}^{* * *}\end{array}$ & $\begin{array}{l}1.17 \\
(0.45) \\
{[0.01]^{* * *}} \\
\{0.02\}^{* *}\end{array}$ & $\begin{array}{l}0.38 \\
(0.14) \\
{[0.01]^{* * *}} \\
\{0.02\}^{* *}\end{array}$ & $\begin{array}{c}0.06 \\
(0.05) \\
{[0.18]} \\
\{0.18\}\end{array}$ \\
\hline $\begin{array}{l}\text { Control mean (follow-up) } \\
\text { Observations }\end{array}$ & $\begin{array}{c}271.97 \\
3,608\end{array}$ & $\begin{array}{l}58.85 \\
3,608\end{array}$ & $\begin{array}{l}37.96 \\
3,608\end{array}$ & $\begin{array}{l}20.86 \\
3,608\end{array}$ & $\begin{array}{l}10.81 \\
3,608\end{array}$ & $\begin{array}{c}7.62 \\
3,608\end{array}$ & $\begin{array}{c}5.36 \\
3,608\end{array}$ & $\begin{array}{c}3.73 \\
3,608\end{array}$ & $\begin{array}{c}0.10 \\
3,608\end{array}$ \\
\hline
\end{tabular}

Note: In this table we report the intent-to-treat estimates of the combined treatment on primary outcomes, obtained by least-squares estimation. Below each coefficient, we report a standard error in parenthesis, a $p$-value in brackets, and a $q$-value in curly braces. Standard errors allow for clustering at the level of the individual. $q$-values are obtained using the sharpened procedure of (Benjamini et al., 2006). We denote significance using $*$ for $10 \%, * *$ for $5 \%$ and $* * *$ for $1 \%$.

Below we report the effect of treatment on savings-related outcomes.

Table A.6: Treatment effects: Attitudes about saving

\begin{tabular}{|c|c|c|c|c|c|c|c|c|}
\hline & $\begin{array}{c}(1) \\
\text { Savings } \\
\text { problems }\end{array}$ & $\begin{array}{c}\text { (2) } \\
\text { Unecessary } \\
\text { purchases }\end{array}$ & $\begin{array}{l}\text { (3) } \\
\text { Pressure } \\
\text { to share }\end{array}$ & $\begin{array}{l}(4) \\
\text { Other: sav } \\
\text { prob }\end{array}$ & $\begin{array}{c}\text { (5) } \\
\text { Other: } \\
\text { unecess purch }\end{array}$ & $\begin{array}{c}(6) \\
\text { Good: money } \\
\text { tracking }\end{array}$ & $\begin{array}{c}(7) \\
\text { Expect: } \\
\text { better(1mth) }\end{array}$ & $\begin{array}{c}(8) \\
\text { Expect: } \\
\text { better(1yr) }\end{array}$ \\
\hline Assignment & $\begin{array}{l}-0.01 \\
(0.01) \\
{[0.34]} \\
\{0.83\}\end{array}$ & $\begin{array}{l}-0.02 \\
(0.01) \\
{[0.27]} \\
\{0.83\}\end{array}$ & $\begin{array}{c}0.01 \\
(0.01) \\
{[0.16]} \\
\{0.83\}\end{array}$ & $\begin{array}{l}-0.02 \\
(0.01) \\
{[0.19]} \\
\{0.83\}\end{array}$ & $\begin{array}{l}-0.01 \\
(0.02) \\
{[0.61]} \\
\{0.83\}\end{array}$ & $\begin{array}{c}0.00 \\
(0.02) \\
{[0.93]} \\
\{0.83\}\end{array}$ & $\begin{array}{l}-0.02 \\
(0.02) \\
{[0.29]} \\
\{0.83\}\end{array}$ & $\begin{array}{l}-0.02 \\
(0.02) \\
{[0.19]} \\
\{0.83\}\end{array}$ \\
\hline $\begin{array}{l}\text { Control mean (follow-up) } \\
\text { Observations }\end{array}$ & $\begin{array}{c}0.35 \\
3,608\end{array}$ & $\begin{array}{c}0.18 \\
3,608\end{array}$ & $\begin{array}{c}0.12 \\
3,608\end{array}$ & $\begin{array}{c}0.30 \\
3,608\end{array}$ & $\begin{array}{c}0.20 \\
3,608\end{array}$ & $\begin{array}{c}0.49 \\
3,608\end{array}$ & $\begin{array}{c}0.46 \\
3,608\end{array}$ & $\begin{array}{c}0.48 \\
3,608\end{array}$ \\
\hline
\end{tabular}

Note: In this table we report the intent-to-treat estimates of the combined treatment on primary outcomes, obtained by least-squares estimation. Below each coefficient, we report a standard error in parenthesis, a $p$-value in brackets, and a $q$-value in curly braces. Standard errors allow for clustering at the level of the individual. $q$-values are obtained using the sharpened procedure of (Benjamini et al., 2006). We denote significance using $*$ for $10 \%, * *$ for $5 \%$ and $* * *$ for $1 \%$. 
Here we investigate if there is any impact of treatment on sector of business operation.

Table A.7: Treatment effects: Sector of business operation

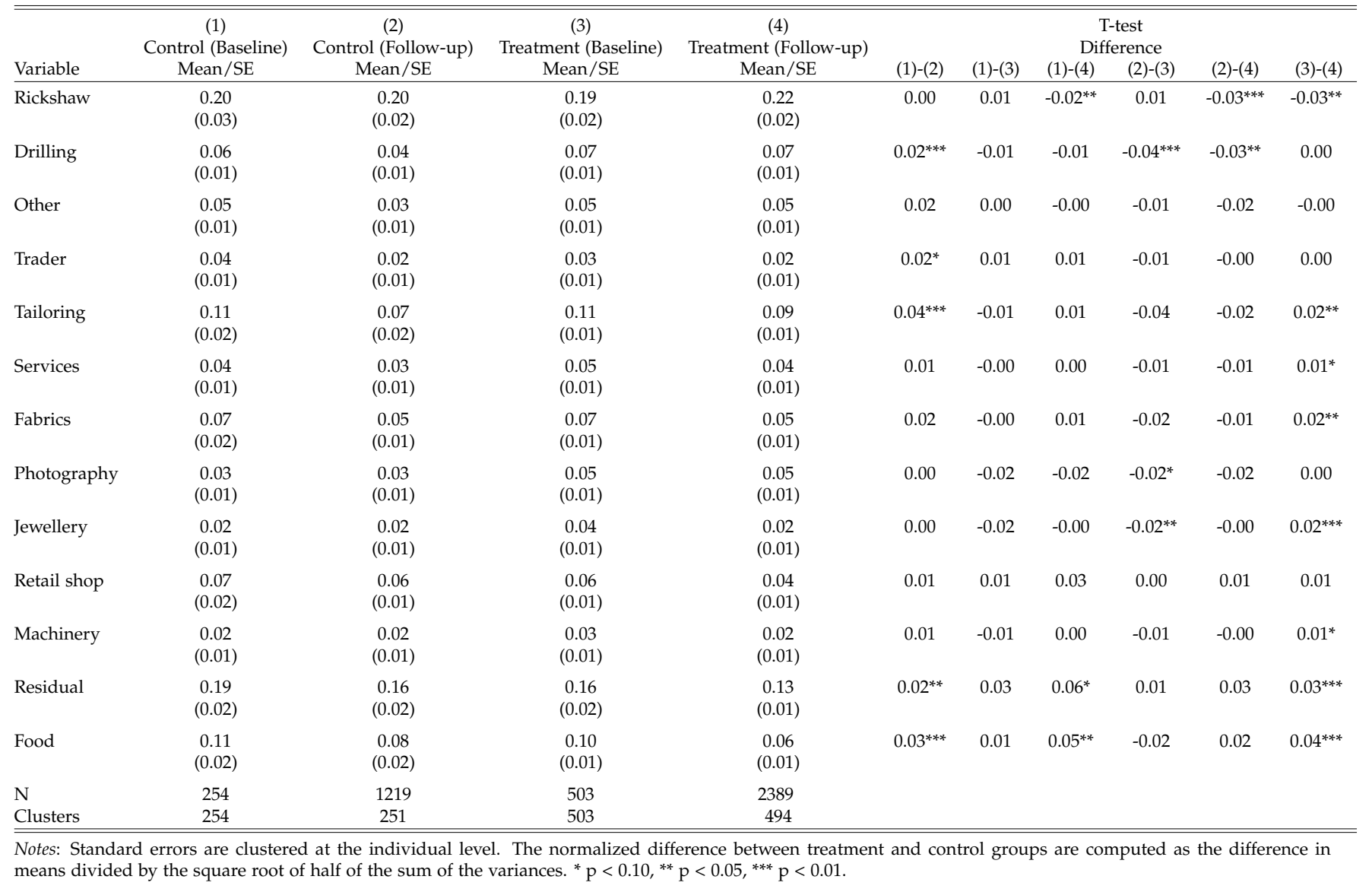

Here we report the effect of treatment on business management practices.

Table A.8: Treatment effects: Microenterprise management practices

\begin{tabular}{lccccc}
\hline & $\begin{array}{c}(1) \\
\text { Management: } \\
\text { overall }\end{array}$ & $\begin{array}{c}(2) \\
\text { Management: } \\
\text { marketing }\end{array}$ & $\begin{array}{c}(3) \\
\text { Management: } \\
\text { buying / stock control }\end{array}$ & $\begin{array}{c}(4) \\
\text { Management: } \\
\text { record keeping }\end{array}$ & $\begin{array}{c}(5) \\
\text { Management: } \\
\text { financial planning }\end{array}$ \\
\hline Assignment & 0.04 & 0.08 & 0.13 & 0.01 & -0.04 \\
& $(0.03)$ & $(0.04)$ & $(0.04)$ & $(0.03)$ & $(0.02)$ \\
& {$[0.25]$} & {$[0.05]^{*}$} & {$[0.00]^{* * *}$} & {$[0.65]$} & {$[0.13]$} \\
Control mean (follow-up) & $\{0.23\}$ & $\{0.12\}$ & $\{0.00\}^{* * *}$ & $\{0.35\}$ & $\{0.15\}$ \\
Observations & 0.00 & 0.00 & 0.00 & 0.00 & 0.00 \\
\hline
\end{tabular}

Note: In this table we report the intent-to-treat estimates of the combined treatment on primary outcomes, obtained by least-squares estimation. Below each coefficient, we report a standard error in parenthesis, a $p$-value in brackets, and a $q$-value in curly braces. Standard errors allow for clustering at the level of the individual. $q$-values are obtained using the sharpened procedure of (Benjamini et al., 2006). We denote significance using $*$ for $10 \%, * *$ for $5 \%$ and $* * *$ for $1 \%$. 


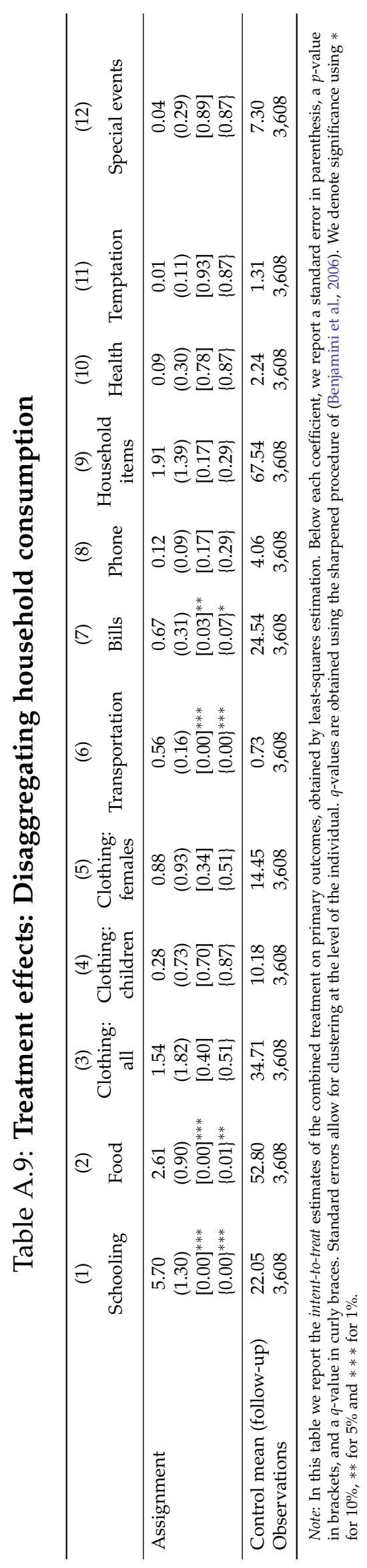


Here, we provide a disaggregation of household educational expenditure, focusing on spending for girls and boys.

\section{Table A.10: Impacts on children's education: extensive margin and overall expenditure}

\begin{tabular}{lcccc}
\hline & $\begin{array}{c}(1) \\
\text { In school: } \\
\text { girls }\end{array}$ & $\begin{array}{c}(2) \\
\text { In school: } \\
\text { boys }\end{array}$ & $\begin{array}{c}\text { Expenditure: } \\
\text { girls }\end{array}$ & $\begin{array}{c}(4) \\
\text { Expenditure: } \\
\text { boys }\end{array}$ \\
\hline Assignment & 0.04 & 0.04 & 4.83 & 3.13 \\
& $(0.03)$ & $(0.03)$ & $(1.31)$ & $(1.32)$ \\
& {$[0.16]$} & {$[0.24]$} & {$[0.00]^{* * *}$} & {$[0.02]^{* *}$} \\
& $\{0.12\}$ & $\{0.14\}$ & $\{0.00\}^{* * *}$ & $\{0.03\}^{* *}$ \\
\hline Control mean (follow-up) & 0.83 & 0.85 & 19.55 & 18.81 \\
Observations & 549 & 487 & 549 & 487 \\
\hline
\end{tabular}

Note: In this table we report the intent-to-treat estimates of the combined treatment on primary outcomes, obtained by least-squares estimation. Below each coefficient, we report a standard error in parenthesis, a $p$-value in brackets, and a $q$-value in curly braces. Standard errors allow for clustering at the level of the individual. $q$-values are obtained using the sharpened procedure of (Benjamini et al., 2006). We denote significance using $*$ for $10 \%, * *$ for $5 \%$ and $* * *$ for $1 \%$.

Table A.11: Disaggregating educational expenditure on children

\begin{tabular}{lcccccccc}
\hline & $\begin{array}{c}(1) \\
\text { School } \\
\text { fees: } \\
\text { girls }\end{array}$ & $\begin{array}{c}\text { School } \\
\text { fees: } \\
\text { boys }\end{array}$ & $\begin{array}{c}\text { Books \& } \\
\text { materials: } \\
\text { girls }\end{array}$ & $\begin{array}{c}\text { Books \& } \\
\text { materials: } \\
\text { boys }\end{array}$ & $\begin{array}{c}(5) \\
\text { expenditure: } \\
\text { girls }\end{array}$ & $\begin{array}{c}\text { Food } \\
\text { expenditure: } \\
\text { boys }\end{array}$ & $\begin{array}{c}\text { Transport } \\
\text { costs: } \\
\text { girls }\end{array}$ & $\begin{array}{c}\text { Transport } \\
\text { costs: } \\
\text { boys }\end{array}$ \\
\hline Assignment & 1.53 & 0.89 & 0.81 & 0.50 & 0.99 & 1.23 & 1.46 & 0.67 \\
& $(0.80)$ & $(0.77)$ & $(0.25)$ & $(0.26)$ & $(0.34)$ & $(0.35)$ & $(0.39)$ & $(0.35)$ \\
& {$[0.06]^{* *}$} & {$[0.25]$} & {$[0.00]^{* * *}$} & {$[0.06]^{*}$} & {$[0.00]^{* * *}$} & {$[0.00]^{* * *}$} & {$[0.00]^{* * *}$} & {$[0.06]^{*}$} \\
& $\{0.03\}^{* *}$ & $\{0.07\}^{*}$ & $\{0.00\}^{* * *}$ & $\{0.03\}^{* *}$ & $\{0.00\}^{* * *}$ & $\{0.00\}^{* * *}$ & $\{0.00\}^{* * *}$ & $\{0.03\}^{* *}$ \\
\hline Control mean (follow-up) & 11.43 & 10.34 & 3.25 & 3.56 & 2.69 & 2.97 & 2.83 & 2.77 \\
Observations & 553 & 493 & 553 & 493 & 553 & 493 & 553 & 493 \\
\hline
\end{tabular}

Note: In this table we report the intent-to-treat estimates of the combined treatment on primary outcomes, obtained by least-squares estimation. Below each coefficient, we report a standard error in parenthesis, a $p$-value in brackets, and a $q$-value in curly braces. Standard errors allow for clustering at the level of the individual. $q$-values are obtained using the sharpened procedure of (Benjamini et al., 2006). We denote significance using $*$ for $10 \%$, $* *$ for $5 \%$ and $* * *$ for $1 \%$

Table A.12: Correlation between educational expenditure and business characteristics

\begin{tabular}{lcccccccc}
\hline \hline & $(1)$ & $(2)$ & $(3)$ & $(4)$ & $(5)$ & $(6)$ & $(7)$ & $(8)$ \\
\hline Total fixed assets & $0.16^{* * *}$ & $0.17^{* * *}$ & $0.15^{* * *}$ & $0.04^{* *}$ & $0.05^{* *}$ & $0.05^{* *}$ & $0.05^{* *}$ & 0.01 \\
& $(0.047)$ & $(0.049)$ & $(0.048)$ & $(0.017)$ & $(0.021)$ & $(0.021)$ & $(0.022)$ & $(0.006)$ \\
Total current assets & & -0.03 & -0.04 & -0.03 & & -0.02 & -0.02 & -0.01 \\
& & $(0.038)$ & $(0.038)$ & $(0.019)$ & & $(0.031)$ & $(0.032)$ & $(0.010)$ \\
Business profits & & & $0.08^{*}$ & -0.01 & & & 0.05 & -0.01 \\
& & & $(0.041)$ & $(0.020)$ & & & $(0.086)$ & $(0.025)$ \\
\hline Observations & 712 & 712 & 712 & 712 & 712 & 712 & 712 & 712 \\
Transformation & Z-score & Z-score & Z-score & Z-score & Log & Log & Log & Log \\
\hline \hline
\end{tabular}

Note: In this table we investigate the correlation (at baseline) between household expenditure on education and business characteristics at baseline (the stock of fixed and current assets, and monthly profits). In columns 1 - 3, we use a z-score transformation for all variables, and in columns 5 - 7 we use a log transformation, adding a one to all values, to prevent the loss of observations with no expenditure (in columns 4 and 8 the dependent variable is a dummy for whether there was any household expenditure on education). Standard errors, reported below each coefficient in parenthesis, allow for clustering at the level of the individual. We denote significance using $*$ for $10 \%, * *$ for $5 \%$ and $* * *$ for $1 \%$. 
Figure A.3: Empirical CDFs for business total fixed assets and business profits

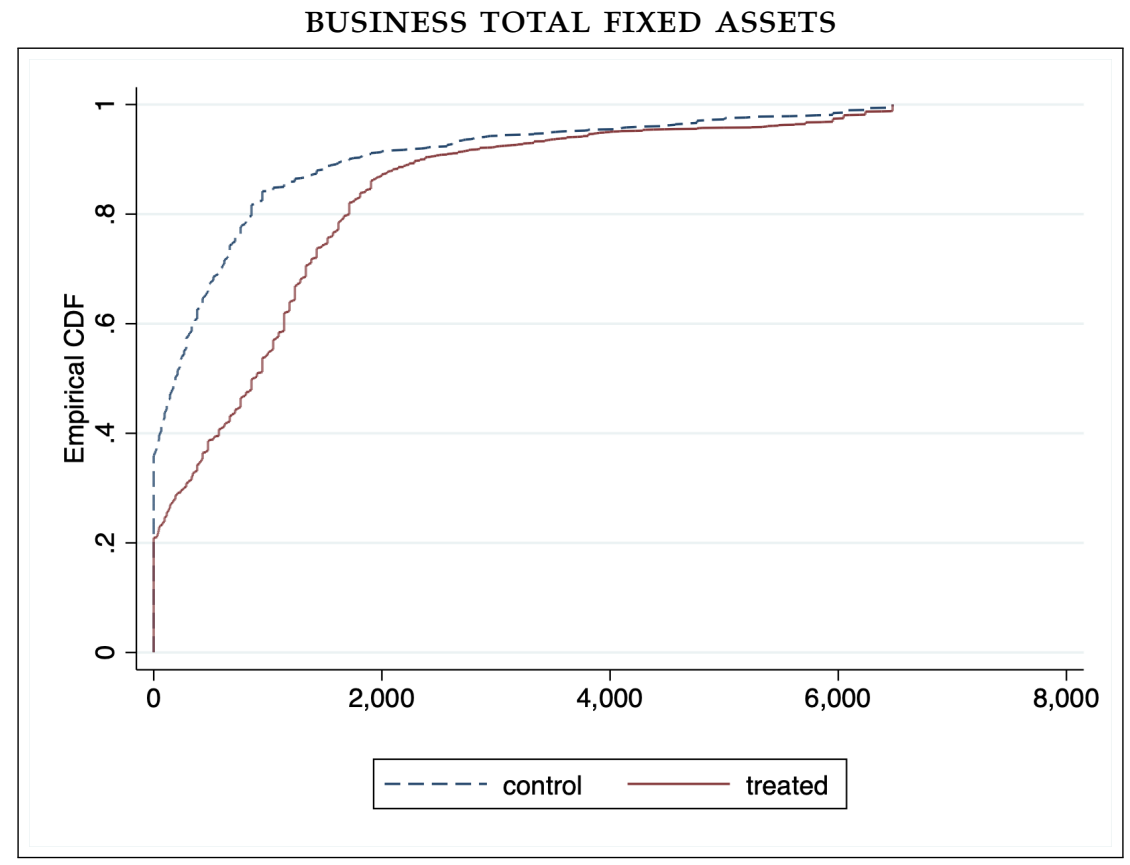

BUSINESS PROFITS

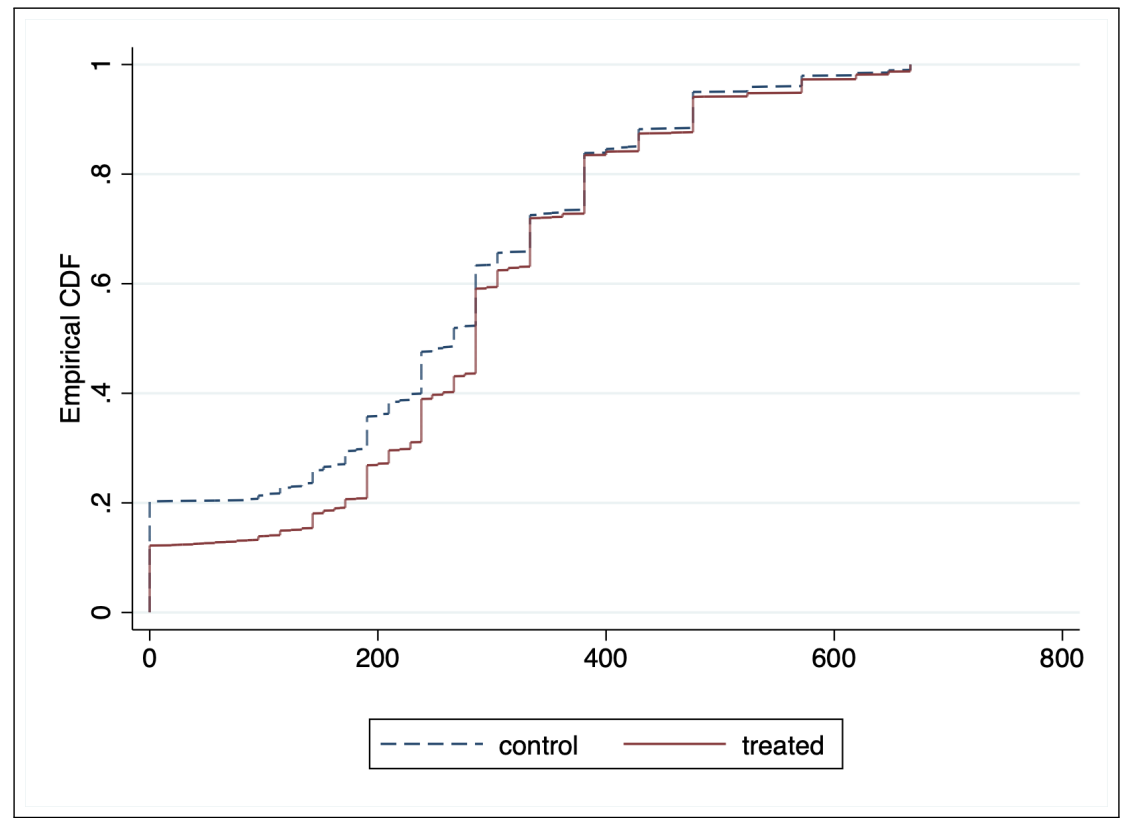

Note: In the above empirical CDFs for business total fixed assets and business profits, we pool all follow-up survey waves. 


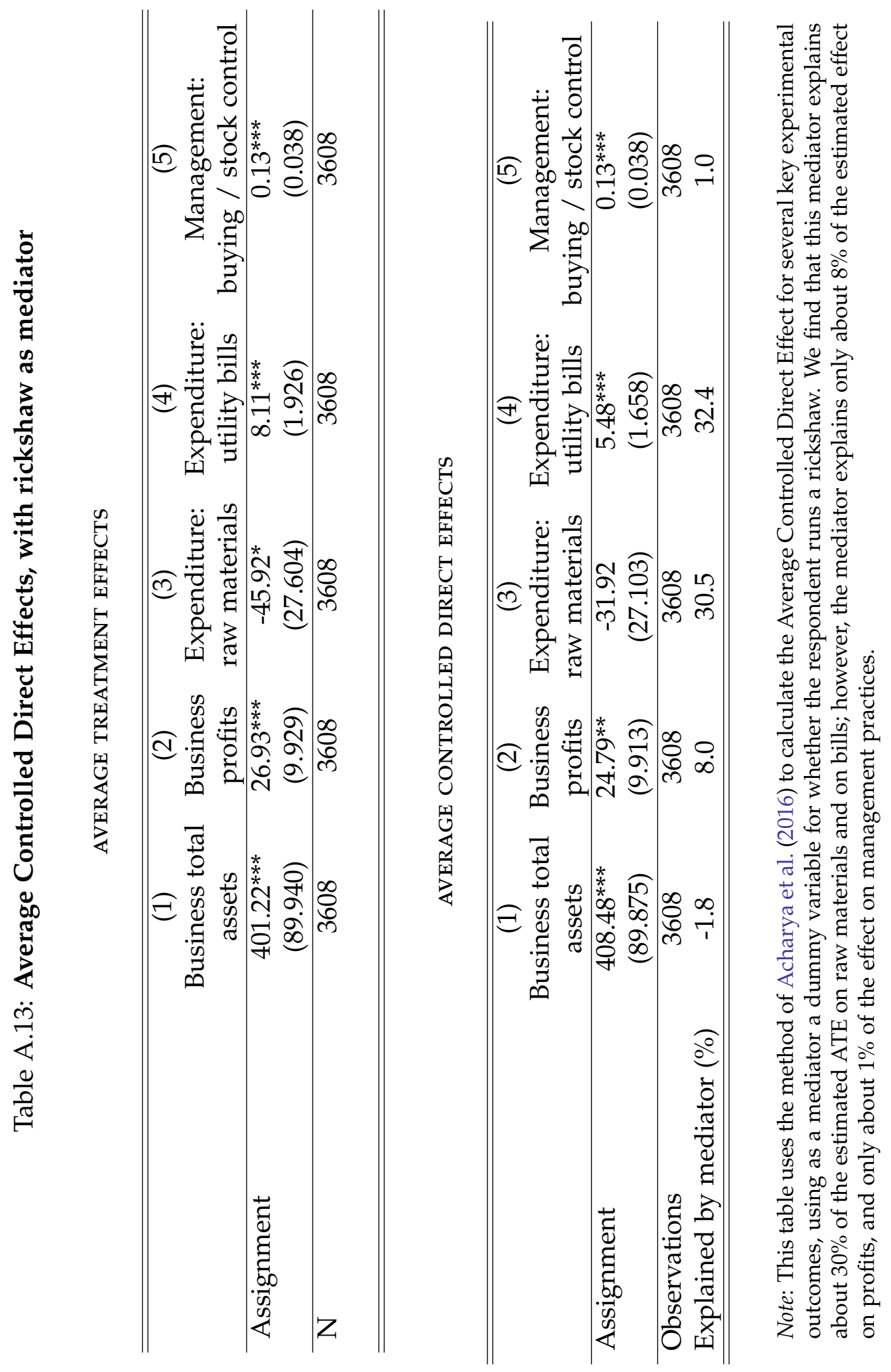




\section{H LATE estimates for pooled treatments}

In this section, we present equivalent local average treatment effect (LATE) estimates to the main ITT regressions, following our pre-analysis plan. ${ }^{4}$ To obtain the LATE estimates, we instrument take-up with treatment, as follows:

$$
\begin{aligned}
& y_{i t}=\beta_{0}+\beta_{1} \cdot \mathrm{A}_{i}+\beta_{2} \cdot y_{i 0}+\phi_{s_{i}}+\varepsilon_{i t} \\
& A_{i}=\alpha_{0}+\alpha_{1} \cdot \mathrm{T}_{i}+\alpha_{2} \cdot y_{i 0}+\psi_{s_{i}}+\mu_{i} .
\end{aligned}
$$

Table A.14: Treatment effects: Primary business outcomes

\begin{tabular}{lcccccc}
\hline & $\begin{array}{c}(1) \\
\text { Runs a } \\
\text { buiness }\end{array}$ & $\begin{array}{c}(2) \\
\text { Number of } \\
\text { businesses }\end{array}$ & $\begin{array}{c}(3) \\
\text { Business } \\
\text { total assets }\end{array}$ & $\begin{array}{c}(4) \\
\text { Business } \\
\text { revenue }\end{array}$ & $\begin{array}{c}(5) \\
\text { Business } \\
\text { profits }\end{array}$ & $\begin{array}{c}(6) \\
\text { Business } \\
\text { employees }\end{array}$ \\
\hline Take-up & 0.16 & 0.17 & 726.21 & 3.29 & 49.06 & 0.06 \\
& $(0.04)$ & $(0.04)$ & $(159.93)$ & $(71.76)$ & $(17.94)$ & $(0.10)$ \\
& {$[0.00]^{* * *}$} & {$[0.00]^{* * *}$} & {$[0.00]^{* * *}$} & {$[0.96]$} & {$[0.01]^{* * *}$} & {$[0.54]$} \\
& $\{0.00\}^{* * *}$ & $\{0.00\}^{* * *}$ & $\{0.00\}^{* * *}$ & $\{0.47\}$ & $\{0.00\}^{* * *}$ & $\{0.28\}$ \\
\hline Control mean (follow-up) & 0.80 & 0.82 & 1003.34 & 689.65 & 249.31 & 0.56 \\
Observations & 3,608 & 3,608 & 3,608 & 3,608 & 3,608 & 3,608 \\
\hline
\end{tabular}

Note: In this table we report the LATE estimates of the combined treatment on primary outcomes, obtained by least-squares estimation. Below each coefficient, we report a standard error in parenthesis, a $p$-value in brackets, and a $q$-value in curly braces. Standard errors allow for clustering at the level of the individual. $q$-values are obtained using the sharpened procedure of (Benjamini et al., 2006). We denote significance using $*$ for $10 \%, * *$ for $5 \%$ and $* * *$ for $1 \%$.

Table A.15: Treatment effects: Effect on business assets

\begin{tabular}{lcccc}
\hline & $\begin{array}{c}(1) \\
\text { Total } \\
\text { fixed assets }\end{array}$ & $\begin{array}{c}\text { Current assets: } \\
\text { cash }\end{array}$ & $\begin{array}{c}(3) \\
\text { Current assets: } \\
\text { accounts receivable }\end{array}$ & $\begin{array}{c}\text { Current assets: } \\
\text { inventory }\end{array}$ \\
\hline Take-up & 793.59 & 4.86 & -1.06 & -53.85 \\
& $(116.27)$ & $(3.20)$ & $(2.65)$ & $(62.36)$ \\
& {$[0.00]^{* * *}$} & {$[0.13]$} & {$[0.69]$} & {$[0.39]$} \\
& $\{0.00\}^{* * *}$ & $\{0.24\}$ & $\{0.53\}$ & $\{0.35\}$ \\
\hline Control mean (follow-up) & 660.19 & 31.38 & 9.93 & 250.77 \\
Observations & 3,608 & 3,608 & 3,608 & 3,608 \\
\hline
\end{tabular}

Note: In this table we report the LATE estimates of the combined treatment on primary outcomes, obtained by leastsquares estimation. Below each coefficient, we report a standard error in parenthesis, a $p$-value in brackets, and a $q$-value in curly braces. Standard errors allow for clustering at the level of the individual. $q$-values are obtained using the sharpened procedure of (Benjamini et al., 2006). We denote significance using $*$ for $10 \%, * *$ for $5 \%$ and $* * *$ for $1 \%$.

\footnotetext{
${ }^{4}$ Available at www.socialscienceregistry.org/trials/3886.
} 
Table A.16: Treatment effects: Business costs

\begin{tabular}{lccccccccc}
\hline & $\begin{array}{c}(1) \\
\text { Raw } \\
\text { materials }\end{array}$ & $\begin{array}{c}(2) \\
\text { Wages }\end{array}$ & $\begin{array}{c}\text { Utility } \\
\text { bills }\end{array}$ & $\begin{array}{c}(4) \\
\text { Rent: } \\
\text { land }\end{array}$ & $\begin{array}{c}(5) \\
\text { Transport }\end{array}$ & $\begin{array}{c}(6) \\
\text { Rent: } \\
\text { machines }\end{array}$ & $\begin{array}{c}(7) \\
\text { Repairs }\end{array}$ & $\begin{array}{c}(8) \\
\text { Phone }\end{array}$ & $\begin{array}{c}(9) \\
\text { Loan } \\
\text { repayment }\end{array}$ \\
\hline Take-up & -82.97 & 7.23 & 14.63 & 4.33 & -0.41 & -5.67 & 2.11 & 0.69 & 0.12 \\
& $(49.93)$ & $(11.15)$ & $(3.41)$ & $(3.71)$ & $(1.61)$ & $(1.67)$ & $(0.81)$ & $(0.25)$ & $(0.09)$ \\
& {$[0.10]^{*}$} & {$[0.52]$} & {$[0.00]^{* *}$} & {$[0.24]$} & {$[0.80]$} & {$[0.00]^{* * *}$} & {$[0.01]^{* * *}$} & {$[0.01]^{* * *}$} & {$[0.18]$} \\
& $\{0.11\}$ & $\{0.35\}$ & $\{0.00\}^{* * *}$ & $\{0.21\}$ & $\{0.37\}$ & $\{0.00\}^{* * *}$ & $\{0.02\}^{* *}$ & $\{0.02\}^{* *}$ & $\{0.18\}$ \\
\hline Control mean (follow-up) & 271.97 & 58.85 & 37.96 & 20.86 & 10.81 & 7.62 & 5.36 & 3.73 & 0.10 \\
Observations & 3,608 & 3,608 & 3,608 & 3,608 & 3,608 & 3,608 & 3,608 & 3,608 & 3,608 \\
\hline
\end{tabular}

Note: In this table we report the LATE estimates of the combined treatment on primary outcomes, obtained by least-squares estimation. Below each coefficient, we report a standard error in parenthesis, a $p$-value in brackets, and a $q$-value in curly braces. Standard errors allow for clustering at the level of the individual. $q$-values are obtained using the sharpened procedure of (Benjamini et al., 2006). We denote significance using $*$ for $10 \%, * *$ for $5 \%$ and $* * *$ for $1 \%$.

Table A.17: Treatment effects: Effect on the household

\begin{tabular}{lccccc}
\hline & $\begin{array}{c}(1) \\
\text { Household } \\
\text { income }\end{array}$ & $\begin{array}{c}\text { Household consumption } \\
\text { expenditure }\end{array}$ & $\begin{array}{c}(3) \\
\text { Household } \\
\text { savings }\end{array}$ & $\begin{array}{c}\text { Household } \\
\text { loans }\end{array}$ & $\begin{array}{c}\text { Household } \\
\text { assets }\end{array}$ \\
\hline Take-up & 56.96 & 23.53 & 29.76 & -41.16 & 36.92 \\
& $(22.72)$ & $(6.15)$ & $(34.71)$ & $(6.45)$ & $(25.52)$ \\
& {$[0.01]^{* *}$} & {$[0.00]^{* * *}$} & {$[0.39]$} & {$[0.00]^{* * *}$} & {$[0.15]$} \\
& $\{0.01\}^{* *}$ & $\{0.00\}^{* * *}$ & $\{0.19\}$ & $\{0.00\}^{* * *}$ & $\{0.08\}^{*}$ \\
\hline Control mean (follow-up) & 357.35 & 220.40 & 113.03 & 46.05 & 681.79 \\
Observations & 3,608 & 3,608 & 3,608 & 3,608 & 1,410 \\
\hline
\end{tabular}

Note: In this table we report the LATE estimates of the combined treatment on primary outcomes, obtained by least-squares estimation. Below each coefficient, we report a standard error in parenthesis, a $p$-value in brackets, and a $q$-value in curly braces. Standard errors allow for clustering at the level of the individual. $q$-values are obtained using the sharpened procedure of (Benjamini et al., 2006). We denote significance using $*$ for $10 \%, * *$ for $5 \%$ and $* * *$ for $1 \%$. 


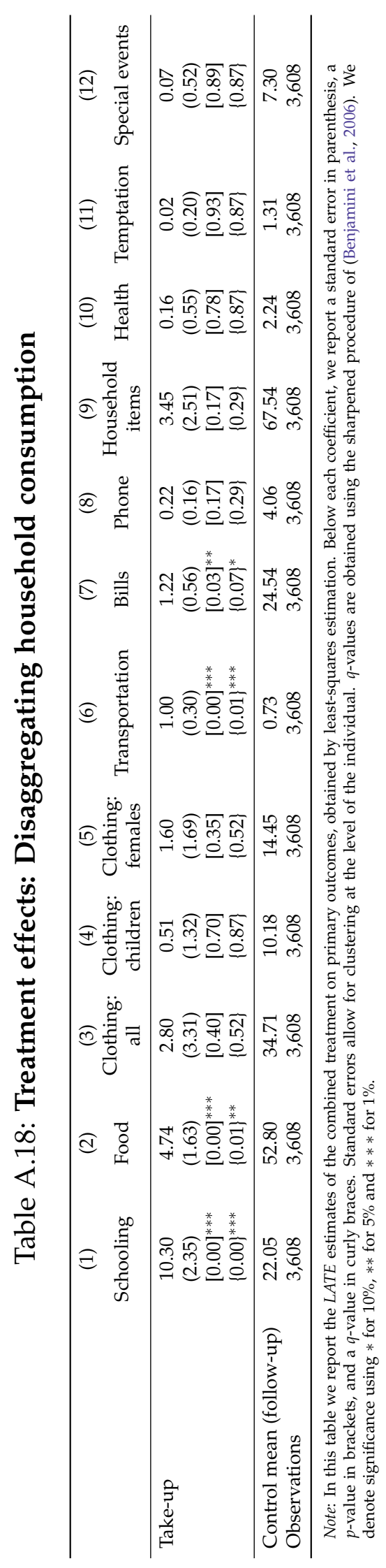


Table A.19: Treatment effects: Microenterprise management practices

\begin{tabular}{lccccc}
\hline & $\begin{array}{c}(1) \\
\text { Management: } \\
\text { overall }\end{array}$ & $\begin{array}{c}(2) \\
\text { Management: } \\
\text { marketing }\end{array}$ & $\begin{array}{c}(3) \\
\text { Management: } \\
\text { buying / stock control }\end{array}$ & $\begin{array}{c}(4) \\
\text { Management: } \\
\text { record keeping }\end{array}$ & $\begin{array}{c}(5) \\
\text { Management: } \\
\text { financial planning }\end{array}$ \\
\hline Take-up & 0.07 & 0.15 & 0.23 & 0.02 & -0.07 \\
& $(0.06)$ & $(0.08)$ & $(0.07)$ & $(0.05)$ & $(0.04)$ \\
& {$[0.25]$} & {$[0.05]^{*}$} & {$[0.00]^{* * *}$} & {$[0.65]$} & {$[0.13]$} \\
Control mean (follow-up) & $\{0.23\}$ & $\{0.12\}$ & $\{0.00\}^{* * *}$ & $\{0.35\}$ & $\{0.16\}$ \\
Observations & 0.00 & 0.00 & 0.00 & 0.00 & 0.00 \\
\hline
\end{tabular}

Note: In this table we report the LATE estimates of the combined treatment on primary outcomes, obtained by least-squares estimation. Below each coefficient, we report a standard error in parenthesis, a $p$-value in brackets, and a $q$-value in curly braces. Standard errors allow for clustering at the level of the individual. $q$-values are obtained using the sharpened procedure of (Benjamini et al., 2006). We denote significance using $*$ for $10 \%, * *$ for $5 \%$ and $* * *$ for $1 \%$.

Table A.20: Treatment effects: Wage work

\begin{tabular}{lcccc}
\hline & $\begin{array}{c}(1) \\
\text { Has a } \\
\text { wage job }\end{array}$ & $\begin{array}{c}(2) \\
\text { Number of } \\
\text { wage jobs }\end{array}$ & $\begin{array}{c}(3) \\
\text { Total } \\
\text { wage hours }\end{array}$ & $\begin{array}{c}(4) \\
\text { Total } \\
\text { wage income }\end{array}$ \\
\hline Take-up & -0.12 & -0.12 & -6.56 & -27.68 \\
& $(0.05)$ & $(0.05)$ & $(2.36)$ & $(10.87)$ \\
& {$[0.01]^{* * *}$} & {$[0.01]^{* *}$} & {$[0.01]^{* * *}$} & {$[0.01]^{* *}$} \\
& $\{0.01\}^{* *}$ & $\{0.01\}^{* *}$ & $\{0.01\}^{* *}$ & $\{0.01\}^{* *}$ \\
\hline Control mean (follow-up) & 0.25 & 0.25 & 12.48 & 55.38 \\
Observations & 3,608 & 3,608 & 3,608 & 3,608 \\
\hline
\end{tabular}

Note: In this table we report the LATE estimates of the combined treatment on primary outcomes, obtained by least-squares estimation. Below each coefficient, we report a standard error in parenthesis, a $p$-value in brackets, and a $q$-value in curly braces. Standard errors allow for clustering at the level of the individual. $q$-values are obtained using the sharpened procedure of (Benjamini et al., 2006). We denote significance using $*$ for $10 \%, * *$ for $5 \%$ and $* * *$ for $1 \%$.

Table A.21: Treatment effects: Attitudes about saving

\begin{tabular}{lcccccccc}
\hline & $\begin{array}{c}(1) \\
\text { Savings } \\
\text { problems }\end{array}$ & $\begin{array}{c}(2) \\
\text { Unecessary } \\
\text { purchases }\end{array}$ & $\begin{array}{c}\text { Pressure } \\
\text { to share }\end{array}$ & $\begin{array}{c}(3) \\
\text { Other: sav } \\
\text { prob }\end{array}$ & $\begin{array}{c}(5) \\
\text { Other: } \\
\text { unecess purch }\end{array}$ & $\begin{array}{c}\text { Good: money } \\
\text { tracking }\end{array}$ & $\begin{array}{c}(7) \\
\text { Expect: } \\
\text { better(1mth) }\end{array}$ & $\begin{array}{c}\text { Expect: } \\
\text { better(1yr) }\end{array}$ \\
\hline Take-up & -0.02 & -0.03 & 0.03 & -0.03 & -0.01 & 0.00 & -0.04 & -0.04 \\
& $(0.03)$ & $(0.03)$ & $(0.02)$ & $(0.03)$ & $(0.03)$ & $(0.03)$ & $(0.04)$ & $(0.03)$ \\
& {$[0.34]$} & {$[0.27]$} & {$[0.16]$} & {$[0.19]$} & {$[0.61]$} & {$[0.93]$} & {$[0.29]$} & {$[0.19]$} \\
& $\{0.82\}$ & $\{0.82\}$ & $\{0.82\}$ & $\{0.82\}$ & $\{0.82\}$ & $\{0.82\}$ & $\{0.82\}$ & $\{0.82\}$ \\
\hline Control mean (follow-up) & 0.35 & 0.18 & 0.12 & 0.30 & 0.20 & 0.49 & 0.46 & 0.48 \\
Observations & 3,608 & 3,608 & 3,608 & 3,608 & 3,608 & 3,608 & 3,608 & 3,608 \\
\hline
\end{tabular}

Note: In this table we report the LATE estimates of the combined treatment on primary outcomes, obtained by least-squares estimation. Below each coefficient, we report a standard error in parenthesis, a $p$-value in brackets, and a $q$-value in curly braces. Standard errors allow for clustering at the level of the individual. $q$-values are obtained using the sharpened procedure of (Benjamini et al., 2006). We denote significance using $*$ for $10 \%, * *$ for $5 \%$ and $* * *$ for $1 \%$. 


\section{ITT and LATE estimates, separating treatment 1 and treat- ment 2}

To estimate the separate ATE of treatment 1 and treatment 2, we estimate:

$$
y_{i t}=\beta_{0}+\beta_{1} \cdot \mathrm{T} 1_{i}+\beta_{2} \cdot \mathrm{T} 2_{i}+\beta_{3} \cdot y_{i 0}+\tau_{s_{i}}+\varepsilon_{i t} .
$$

To estimate the separate LATE of treatment 1 and treatment 2, we instrument take-up with treatment as follows:

$$
\begin{aligned}
y_{i t} & =\beta_{0}+\beta_{1} \cdot \mathrm{A} 1_{i}+\beta_{2} \cdot \mathrm{A} 2_{i}+\beta_{3} \cdot y_{i 0}+\tau_{s_{i}}+\varepsilon_{i t} \\
\mathrm{~A} 1_{i} & =\gamma_{0}+\gamma_{1} \cdot \mathrm{T} 1_{i}+\gamma_{2} \cdot \mathrm{T} 2_{i}+\gamma_{3} \cdot y_{i 0}+\phi_{s_{i}}+\mu_{i} \\
\mathrm{~A} 2_{i} & =\delta_{0}+\delta_{1} \cdot \mathrm{T} 1_{i}+\delta_{2} \cdot \mathrm{T} 2_{i}+\delta_{3} \cdot y_{i 0}+\omega_{s_{i}}+v_{i}
\end{aligned}
$$

Table A.22: Treatment effects (ITT): Primary business outcomes

\begin{tabular}{lcccccc}
\hline \hline & $\begin{array}{c}(1) \\
\text { Runs a } \\
\text { buiness }\end{array}$ & $\begin{array}{c}(2) \\
\text { Number of } \\
\text { businesses }\end{array}$ & $\begin{array}{c}(3) \\
\text { Business } \\
\text { total assets }\end{array}$ & $\begin{array}{c}(4) \\
\text { Business } \\
\text { revenue }\end{array}$ & $\begin{array}{c}(5) \\
\text { Business } \\
\text { profits }\end{array}$ & $\begin{array}{c}\text { (6) } \\
\text { Business } \\
\text { employees }\end{array}$ \\
\hline Assignment 1 & $0.09^{* * *}$ & $0.10^{* * *}$ & $429.78^{* * *}$ & 16.40 & $28.56^{* *}$ & 0.03 \\
& $(0.028)$ & $(0.028)$ & $(105.218)$ & $(45.279)$ & $(11.251)$ & $(0.065)$ \\
Assignment 2 & $0.09^{* * *}$ & $0.09^{* * *}$ & $371.42^{* * *}$ & -13.41 & $25.23^{* *}$ & 0.04 \\
& $(0.028)$ & $(0.029)$ & $(101.270)$ & $(45.180)$ & $(11.205)$ & $(0.067)$ \\
\hline Observations & 3608 & 3608 & 3608 & 3608 & 3608 & 3608 \\
Test: Assignment 1 Assignment 2 & 0.881 & 0.904 & 0.566 & 0.494 & 0.751 & 0.946 \\
Control mean (follow-up) & 0.80 & 0.82 & 1003.34 & 689.65 & 249.31 & 0.56 \\
\hline \hline
\end{tabular}

Note: In this table we report the intent-to-treat estimates of the separated treatments on primary outcomes, obtained by least-squares estimation. Below each coefficient, we report a standard error in parenthesis. We denote significance using $*$ for $10 \%, * *$ for $5 \%$ and $* * *$ for $1 \%$.

Table A.23: Treatment effects (ITT): Effect on business assets

\begin{tabular}{lcccc}
\hline \hline & $\begin{array}{c}(1) \\
\text { Total } \\
\text { fixed assets }\end{array}$ & $\begin{array}{c}\text { Current assets: } \\
\text { cash }\end{array}$ & $\begin{array}{c}(3) \\
\text { Current assets: } \\
\text { accounts receivable }\end{array}$ & $\begin{array}{c}\text { Current assets: } \\
\text { inventory }\end{array}$ \\
\hline Assignment 1 & $480.92^{* * *}$ & 2.14 & 0.11 & -39.44 \\
& $(81.318)$ & $(1.948)$ & $(1.709)$ & $(37.397)$ \\
Assignment 2 & $393.30^{* * *}$ & 3.24 & -1.31 & -19.65 \\
& $(76.763)$ & $(2.132)$ & $(1.624)$ & $(40.589)$ \\
\hline Observations & 3608 & 3608 & 3608 & 3608 \\
Test: Assignment 1 = Assignment 2 & 0.297 & 0.584 & 0.373 & 0.585 \\
Control mean (follow-up) & 660.19 & 31.38 & 9.93 & 250.77 \\
\hline
\end{tabular}

Note: In this table we report the intent-to-treat estimates of the separated treatments on primary outcomes, obtained by least-squares estimation. Below each coefficient, we report a standard error in parenthesis. We denote significance using $*$ for $10 \%, * *$ for $5 \%$ and $* * *$ for $1 \%$. 


\section{Table A.24: Treatment effects (ITT): Microenterprise management practices}

\begin{tabular}{|c|c|c|c|c|c|}
\hline & $\begin{array}{c}(1) \\
\text { Management: } \\
\text { overall }\end{array}$ & $\begin{array}{c}(2) \\
\text { Management: } \\
\text { marketing }\end{array}$ & $\begin{array}{c}(3) \\
\text { Management: } \\
\text { buying / stock control }\end{array}$ & $\begin{array}{c}(4) \\
\text { Management: } \\
\text { record keeping }\end{array}$ & $\begin{array}{c}(5) \\
\text { Management: } \\
\text { financial planning }\end{array}$ \\
\hline \multirow[t]{2}{*}{ Assignment 1} & 0.01 & $0.09^{*}$ & $0.08^{*}$ & -0.01 & $-0.06^{* *}$ \\
\hline & $(0.037)$ & $(0.048)$ & $(0.044)$ & $(0.029)$ & $(0.027)$ \\
\hline \multirow[t]{2}{*}{ Assignment 2} & $0.07^{*}$ & 0.08 & $0.17^{* * *}$ & 0.04 & -0.01 \\
\hline & $(0.038)$ & $(0.049)$ & $(0.043)$ & $(0.029)$ & $(0.029)$ \\
\hline Observations & 3608 & 3608 & 3608 & 3608 & 3608 \\
\hline Test: Assignment $1=$ Assignment 2 & 0.085 & 0.775 & 0.039 & 0.064 & 0.072 \\
\hline Control mean (follow-up) & -0.00 & 0.00 & 0.00 & -0.00 & -0.00 \\
\hline
\end{tabular}

Note: In this table we report the intent-to-treat estimates of the separated treatments on primary outcomes, obtained by least-squares estimation. Below each coefficient, we report a standard error in parenthesis. We denote significance using $*$ for $10 \%, * *$ for $5 \%$ and $* * *$ for $1 \%$.

\section{Table A.25: Treatment effects (ITT): Effect on the household}

\begin{tabular}{|c|c|c|c|c|c|}
\hline & $\begin{array}{l}\text { (1) } \\
\text { Household } \\
\text { income }\end{array}$ & $\begin{array}{l}\text { (2) } \\
\text { Household consumption } \\
\text { expenditure }\end{array}$ & $\begin{array}{l}\text { (3) } \\
\text { Household } \\
\text { savings }\end{array}$ & $\begin{array}{c}\text { (4) } \\
\text { Household } \\
\text { loans }\end{array}$ & $\begin{array}{l}\text { (5) } \\
\text { Household } \\
\text { assets }\end{array}$ \\
\hline Assignment 1 & $\begin{array}{c}27.30^{*} \\
(14.532)\end{array}$ & $\begin{array}{c}13.14^{* * *} \\
(3.838)\end{array}$ & $\begin{array}{c}-0.83 \\
(23.138)\end{array}$ & $\begin{array}{c}-20.11^{* * *} \\
(4.052)\end{array}$ & $\begin{array}{c}24.80 \\
(16.400)\end{array}$ \\
\hline Assignment 2 & $\begin{array}{l}35.83^{* *} \\
(14.511)\end{array}$ & $\begin{array}{l}12.76^{* * *} \\
(4.035)\end{array}$ & $\begin{array}{c}34.49 \\
(22.878)\end{array}$ & $\begin{array}{c}-25.64^{* * *} \\
(4.108)\end{array}$ & $\begin{array}{c}15.61 \\
(16.166))\end{array}$ \\
\hline Observations & 3608 & 3608 & 3608 & 3608 & 1410 \\
\hline Test: Assignment $1=$ Assignment 2 & 0.549 & 0.926 & 0.166 & 0.128 & 0.579 \\
\hline Control mean (follow-up) & 357.35 & 220.40 & 113.03 & 46.05 & 681.79 \\
\hline
\end{tabular}

Note: In this table we report the intent-to-treat estimates of the separated treatments on primary outcomes, obtained by least-squares estimation. Below each coefficient, we report a standard error in parenthesis. We denote significance using $*$ for $10 \%, * *$ for $5 \%$ and $* * *$ for $1 \%$.

\section{Table A.26: Treatment effects (ITT): Wage work}

\begin{tabular}{lcccc}
\hline \hline & $(1)$ & $(2)$ & $(3)$ & $(4)$ \\
& $\begin{array}{c}\text { Has a } \\
\text { wage job }\end{array}$ & $\begin{array}{c}\text { Number of } \\
\text { wage jobs }\end{array}$ & $\begin{array}{c}\text { Total } \\
\text { wage hours }\end{array}$ & $\begin{array}{c}\text { Total } \\
\text { wage income }\end{array}$ \\
\hline Assignment 1 & $-0.07^{* *}$ & $-0.07^{* *}$ & $-3.93^{* * *}$ & $-17.62^{* * *}$ \\
Assignment 2 & $(0.029)$ & $(0.029)$ & $(1.484)$ & $(6.785)$ \\
& $-0.06^{* *}$ & $-0.06^{* *}$ & $-3.31^{* *}$ & $-12.79^{*}$ \\
& $(0.029)$ & $(0.029)$ & $(1.465)$ & $(6.814)$ \\
\hline Observations & 3608 & 3608 & 3608 & 3608 \\
Test: Assignment 1 = Assignment 2 & 0.678 & 0.678 & 0.650 & 0.451 \\
Control mean (follow-up) & 0.25 & 0.25 & 12.48 & 55.38 \\
\hline \hline
\end{tabular}

Note: In this table we report the intent-to-treat estimates of the separated treatments on primary outcomes, obtained by least-squares estimation. Below each coefficient, we report a standard error in parenthesis. We denote significance using $*$ for $10 \%$, $* *$ for $5 \%$ and $* * *$ for $1 \%$. 
Table A.27: Treatment effects (ITT): Attitudes about saving

\begin{tabular}{|c|c|c|c|c|c|c|c|c|}
\hline & $\begin{array}{c}\text { (1) } \\
\text { Savings } \\
\text { problems }\end{array}$ & $\begin{array}{c}\text { (2) } \\
\text { Unecessary } \\
\text { purchases }\end{array}$ & $\begin{array}{c}\text { (3) } \\
\text { Pressure } \\
\text { to share }\end{array}$ & $\begin{array}{c}\text { (4) } \\
\text { Other: sav } \\
\text { prob }\end{array}$ & $\begin{array}{c}\text { (5) } \\
\text { Other: } \\
\text { unecess purch }\end{array}$ & $\begin{array}{c}(6) \\
\text { Good: money } \\
\text { tracking }\end{array}$ & $\begin{array}{c}\text { (7) } \\
\text { Expect: } \\
\text { better(1mth) }\end{array}$ & $\begin{array}{c}\text { (8) } \\
\text { Expect: } \\
\text { better(1yr) }\end{array}$ \\
\hline Assignment 1 & $\begin{array}{c}-0.02 \\
(0.017)\end{array}$ & $\begin{array}{c}-0.03^{*} \\
(0.015)\end{array}$ & $\begin{array}{c}0.01 \\
(0.012)\end{array}$ & $\begin{array}{c}-0.02 \\
(0.016)\end{array}$ & $\begin{array}{c}-0.02 \\
(0.018)\end{array}$ & $\begin{array}{c}0.01 \\
(0.022)\end{array}$ & $\begin{array}{l}-0.05^{* *} \\
(0.023)\end{array}$ & $\begin{array}{c}-0.02 \\
(0.020)\end{array}$ \\
\hline Assignment 2 & $\begin{array}{c}-0.00 \\
(0.016)\end{array}$ & $\begin{array}{c}-0.00 \\
(0.016)\end{array}$ & $\begin{array}{c}0.02 \\
(0.013)\end{array}$ & $\begin{array}{c}-0.02 \\
(0.017)\end{array}$ & $\begin{array}{c}0.00 \\
(0.018)\end{array}$ & $\begin{array}{c}-0.02 \\
(0.022)\end{array}$ & $\begin{array}{c}0.01 \\
(0.023)\end{array}$ & $\begin{array}{c}-0.02 \\
(0.021)\end{array}$ \\
\hline Observations & 3608 & 3608 & 3608 & 3608 & 3608 & 3608 & 3608 & 3608 \\
\hline Test: Assignment $1=$ Assignment 2 & 0.274 & 0.139 & 0.735 & 0.805 & 0.272 & 0.216 & 0.020 & 0.833 \\
\hline Control mean (follow-up) & 0.35 & 0.18 & 0.12 & 0.30 & 0.20 & 0.49 & 0.46 & 0.48 \\
\hline
\end{tabular}

Note: In this table we report the intent-to-treat estimates of the separated treatments on primary outcomes, obtained by least-squares estimation. Below each coefficient, we report a standard error in parenthesis. We denote significance using $*$ for $10 \%, * *$ for $5 \%$ and $* * *$ for $1 \%$.

Table A.28: Treatment effects (LATE): Primary business outcomes

\begin{tabular}{lccccccc}
\hline \hline & $\begin{array}{c}(1) \\
\text { Runs a } \\
\text { buiness }\end{array}$ & $\begin{array}{c}\text { Number of } \\
\text { businesses }\end{array}$ & $\begin{array}{c}(3) \\
\text { Business } \\
\text { total assets }\end{array}$ & $\begin{array}{c}(4) \\
\text { Business } \\
\text { revenue }\end{array}$ & $\begin{array}{c}\text { Business } \\
\text { profits }\end{array}$ & $\begin{array}{c}(6) \\
\text { Business } \\
\text { employees }\end{array}$ \\
\hline Take-up 1 & $0.17^{* * *}$ & $0.18^{* * *}$ & $812.19^{* * *}$ & 30.86 & $54.46^{* *}$ & $55.06^{* * *}$ & 0.06 \\
& $(0.052)$ & $(0.052)$ & $(195.210)$ & $(85.374)$ & $(21.159)$ & $(21.175)$ & $(0.123)$ \\
Take-up 2 & $0.15^{* * * *}$ & $0.16^{* * *}$ & $614.80^{* * *}$ & -32.62 & $42.14^{* *}$ & $38.29^{*}$ & 0.07 \\
& $(0.051)$ & $(0.052)$ & $(181.478)$ & $(85.002)$ & $(20.514)$ & $(20.655)$ & $(0.126)$ \\
\hline Observations & 3608 & 3608 & 3608 & 3608 & 3608 & 3608 & 3608 \\
Test: Take-up 1 = Take-up 2 & 0.640 & 0.655 & 0.334 & 0.493 & 0.567 & 0.439 & 0.984 \\
Control mean (follow-up) & 0.80 & 0.82 & 1003.34 & 689.65 & 249.31 & 251.69 & 0.56 \\
\hline \hline
\end{tabular}

Note: In this table we report the local average treatment effect estimates of the separated treatments on primary outcomes, obtained by least-squares estimation. Below each coefficient, we report a standard error in parenthesis. We denote significance using $*$ for $10 \%, * *$ for $5 \%$ and $* * *$ for $1 \%$.

Table A.29: Treatment effects (LATE): Effect on business assets

\begin{tabular}{lcccc}
\hline \hline & $\begin{array}{c}\text { Total } \\
\text { fixed assets }\end{array}$ & $\begin{array}{c}\text { Current assets: } \\
\text { cash }\end{array}$ & $\begin{array}{c}\text { Current assets: } \\
\text { accounts receivable }\end{array}$ & $\begin{array}{c}\text { Current assets: } \\
\text { inventory }\end{array}$ \\
\hline Take-up 1 & $909.08^{* * *}$ & 4.05 & 0.20 & -74.36 \\
& $(146.874)$ & $(3.672)$ & $(3.222)$ & $(70.344)$ \\
Take-up 2 & $643.98^{* * *}$ & 5.90 & -2.70 & -27.21 \\
& $(132.682)$ & $(4.007)$ & $(3.038)$ & $(76.187)$ \\
\hline Observations & 3608 & 3608 & 3608 & 3608 \\
Test: Take-up 1 = Take-up 2 & 0.097 & 0.659 & 0.394 & 0.536 \\
Control mean (follow-up) & 660.19 & 31.38 & 9.93 & 250.77 \\
\hline \hline
\end{tabular}

Note: In this table we report the local average treatment effect estimates of the separated treatments on primary outcomes, obtained by least-squares estimation. Below each coefficient, we report a standard error in parenthesis. We denote significance using $*$ for $10 \%, * *$ for $5 \%$ and $* * *$ for $1 \%$. 


\section{Table A.30: Treatment effects (LATE): Microenterprise management practices}

\begin{tabular}{lccccc}
\hline \hline & $\begin{array}{c}(1) \\
\text { Management: } \\
\text { overall }\end{array}$ & $\begin{array}{c}(2) \\
\text { Management: } \\
\text { marketing }\end{array}$ & $\begin{array}{c}(3) \\
\text { Management: } \\
\text { buying / stock control }\end{array}$ & $\begin{array}{c}\text { Management: } \\
\text { record keeping }\end{array}$ & $\begin{array}{c}\text { Management: } \\
\text { financial planning }\end{array}$ \\
\hline Take-up 1 & 0.02 & $0.17^{*}$ & $0.16^{*}$ & -0.02 & $-0.11^{* *}$ \\
Take-up 2 & $(0.070)$ & $(0.090)$ & $(0.082)$ & $(0.055)$ & $(0.051)$ \\
& $0.14^{*}$ & 0.12 & $0.32^{* * *}$ & 0.08 & -0.01 \\
\hline Observations & $(0.070)$ & $(0.093)$ & $(0.082)$ & $(0.055)$ & $(0.054)$ \\
Test: Take-up 1 = Take-up 2 & 3608 & 3608 & 3608 & 3608 & 3608 \\
Control mean (follow-up) & 0.103 & 0.661 & 0.071 & 0.073 & 0.060 \\
\hline \hline
\end{tabular}

Note: In this table we report the local average treatment effect estimates of the separated treatments on primary outcomes, obtained by least-squares estimation. Below each coefficient, we report a standard error in parenthesis. We denote significance using $*$ for $10 \%, * *$ for $5 \%$ and $* * *$ for $1 \%$.

\section{Table A.31: Treatment effects (LATE): Effect on the household}

\begin{tabular}{lcccccc}
\hline \hline & $(1)$ & $(2)$ & $(3)$ & $(4)$ & $(5)$ & $(6)$ \\
& $\begin{array}{c}\text { Household } \\
\text { income }\end{array}$ & $\begin{array}{c}\text { Household consumption } \\
\text { expenditure }\end{array}$ & $\begin{array}{c}\text { Household } \\
\text { savings }\end{array}$ & $\begin{array}{c}\text { Household } \\
\text { loans }\end{array}$ & $\begin{array}{c}\text { Household } \\
\text { assets }\end{array}$ \\
\hline Take-up 1 & $51.42^{*}$ & $24.94^{* * *}$ & -1.51 & $-38.26^{* * *}$ & 46.75 & 1.19 \\
& $(27.099)$ & $(7.281)$ & $(43.600)$ & $(7.479)$ & $(30.907)$ & $(3.200)$ \\
Take-up 2 & $64.15^{* *}$ & $21.71^{* * *}$ & 70.37 & $-44.93^{* * *}$ & 23.66 & -4.18 \\
& $(26.888)$ & $(7.701)$ & $(44.504)$ & $(7.189)$ & $(31.075)$ & $(2.958)$ \\
\hline Observations & 3608 & 3608 & 3608 & 3608 & 1410 & 3608 \\
Test: Take-up 1 = Take-up 2 & 0.666 & 0.705 & 0.187 & 0.351 & 0.514 & 0.134 \\
Control mean (follow-up) & 357.35 & 220.40 & 113.03 & 46.05 & 681.79 & 14.29 \\
\hline \hline
\end{tabular}

Note: In this table we report the local average treatment effect estimates of the separated treatments on primary outcomes, obtained by least-squares estimation. Below each coefficient, we report a standard error in parenthesis. We denote significance using $*$ for $10 \%, * *$ for $5 \%$ and $* * *$ for $1 \%$.

Table A.32: Treatment effects (LATE): Wage work

\begin{tabular}{lcccc}
\hline \hline & $\begin{array}{c}(1) \\
\text { Has a } \\
\text { wage job }\end{array}$ & $\begin{array}{c}\text { Number of } \\
\text { wage jobs }\end{array}$ & $\begin{array}{c}\text { Total } \\
\text { wage hours }\end{array}$ & $\begin{array}{c}\text { Total } \\
\text { wage income }\end{array}$ \\
\hline Take-up 1 & $-0.13^{* *}$ & $-0.13^{* *}$ & $-7.41^{* * *}$ & $-33.29^{* * *}$ \\
& $(0.054)$ & $(0.054)$ & $(2.786)$ & $(12.745)$ \\
Take-up 2 & $-0.10^{*}$ & $-0.10^{*}$ & $-5.46^{* *}$ & -20.38 \\
& $(0.054)$ & $(0.054)$ & $(2.715)$ & $(12.716)$ \\
\hline Observations & 3608 & 3608 & 3608 & 3608 \\
Test: Take-up 1 = Take-up 2 & 0.535 & 0.536 & 0.494 & 0.335 \\
Control mean (follow-up) & 0.25 & 0.25 & 12.48 & 55.38 \\
\hline \hline
\end{tabular}

Note: In this table we report the local average treatment effect estimates of the separated treatments on primary outcomes, obtained by least-squares estimation. Below each coefficient, we report a standard error in parenthesis. We denote significance using $*$ for $10 \%, * *$ for $5 \%$ and $* * *$ for $1 \%$. 
Table A.33: Treatment effects (LATE): Attitudes about saving

\begin{tabular}{|c|c|c|c|c|c|c|c|c|}
\hline & $\begin{array}{c}\text { (1) } \\
\text { Savings } \\
\text { problems }\end{array}$ & $\begin{array}{c}\text { (2) } \\
\text { Unecessary } \\
\text { purchases }\end{array}$ & $\begin{array}{l}\text { (3) } \\
\text { Pressure } \\
\text { to share }\end{array}$ & $\begin{array}{l}\text { (4) } \\
\text { Other: sav } \\
\text { prob }\end{array}$ & $\begin{array}{c}\text { (5) } \\
\text { Other: } \\
\text { unecess purch }\end{array}$ & $\begin{array}{c}(6) \\
\text { Good: money } \\
\text { tracking }\end{array}$ & $\begin{array}{c}\text { (7) } \\
\text { Expect: } \\
\text { better(1mth) }\end{array}$ & $\begin{array}{c}\text { (8) } \\
\text { Expect: } \\
\text { better(1yr) }\end{array}$ \\
\hline Take-up 1 & $\begin{array}{c}-0.04 \\
(0.032)\end{array}$ & $\begin{array}{l}-0.05^{*} \\
(0.029)\end{array}$ & $\begin{array}{c}0.02 \\
(0.023)\end{array}$ & $\begin{array}{c}-0.04 \\
(0.030)\end{array}$ & $\begin{array}{c}-0.03 \\
(0.034)\end{array}$ & $\begin{array}{c}0.02 \\
(0.041)\end{array}$ & $\begin{array}{l}-0.09^{* *} \\
(0.045)\end{array}$ & $\begin{array}{c}-0.04 \\
(0.037)\end{array}$ \\
\hline Take-up 2 & $\begin{array}{c}-0.00 \\
(0.030)\end{array}$ & $\begin{array}{c}0.00 \\
(0.031)\end{array}$ & $\begin{array}{c}0.03 \\
(0.024)\end{array}$ & $\begin{array}{c}-0.03 \\
(0.032)\end{array}$ & $\begin{array}{c}0.01 \\
(0.034)\end{array}$ & $\begin{array}{c}-0.04 \\
(0.042)\end{array}$ & $\begin{array}{c}0.03 \\
(0.045)\end{array}$ & $\begin{array}{c}-0.04 \\
(0.040)\end{array}$ \\
\hline Observations & 3608 & 3608 & 3608 & 3608 & 3608 & 3608 & 3608 & 3608 \\
\hline Test: Take-up 1 = Take-up 2 & 0.249 & 0.119 & 0.813 & 0.736 & 0.258 & 0.220 & 0.019 & 0.908 \\
\hline Control mean (follow-up) & 0.35 & 0.18 & 0.12 & 0.30 & 0.20 & 0.49 & 0.46 & 0.48 \\
\hline
\end{tabular}

Note: In this table we report the local average treatment effect estimates of the separated treatments on primary outcomes, obtained by least-squares estimation. Below each coefficient, we report a standard error in parenthesis. We denote significance using $*$ for $10 \%, * *$ for $5 \%$ and $* * *$ for $1 \%$. 


\section{J Disaggregating results by survey wave}

Here we repeat our earlier ITT analysis, disaggregating by survey wave. Specifically, we show estimates individually for follow-up surveys at the three-month, six-month, 12-month, 18-month and 24-month points.

Table A.34: Disaggregating results by survey wave: business outcomes

PANEL A: THREE-MONTH FOLLOW-UP

\begin{tabular}{|c|c|c|c|c|c|c|c|c|c|c|}
\hline & $\begin{array}{c}\text { (1) } \\
\text { Runs a } \\
\text { buiness }\end{array}$ & $\begin{array}{c}\text { (2) } \\
\text { Number of } \\
\text { businesses }\end{array}$ & $\begin{array}{c}\text { (3) } \\
\text { Business } \\
\text { total assets }\end{array}$ & $\begin{array}{l}\text { (4) } \\
\text { Business } \\
\text { revenue }\end{array}$ & $\begin{array}{l}\text { (5) } \\
\text { Business } \\
\text { profits }\end{array}$ & $\begin{array}{c}\text { (6) } \\
\text { Business } \\
\text { employees }\end{array}$ & $\begin{array}{c}\text { (7) } \\
\text { Total } \\
\text { fixed assets }\end{array}$ & $\begin{array}{c}(8) \\
\text { Current assets: } \\
\text { cash }\end{array}$ & $\begin{array}{c}\text { (9) } \\
\text { Current assets: } \\
\text { accounts receivable }\end{array}$ & $\begin{array}{c}(10) \\
\text { Current assets: } \\
\text { inventory }\end{array}$ \\
\hline Assignment & $\begin{array}{l}0.08^{* * *} \\
(0.027)\end{array}$ & $\begin{array}{l}0.11^{* * *} \\
(0.032)\end{array}$ & $\begin{array}{l}433.23^{* * *} \\
(106.694)\end{array}$ & $\begin{array}{c}-4.77 \\
(47.511)\end{array}$ & $\begin{array}{c}14.54 \\
(10.985)\end{array}$ & $\begin{array}{l}-0.00 \\
(0.074)\end{array}$ & $\begin{array}{c}454.65^{* * *} \\
(76.133)\end{array}$ & $\begin{array}{c}3.35 \\
(4.277)\end{array}$ & $\begin{array}{c}-0.23 \\
(4.515)\end{array}$ & $\begin{array}{c}-3.02 \\
(46.455)\end{array}$ \\
\hline Observations & 737 & 737 & 737 & 737 & 737 & 737 & 737 & 737 & 737 & 737 \\
\hline Control mean (follow-up) & 0.84 & 0.89 & 1149.01 & 674.54 & 238.25 & 0.60 & 710.79 & 44.31 & 18.39 & 303.40 \\
\hline
\end{tabular}

PANEL B: SIX-MONTH FOLLOW-UP

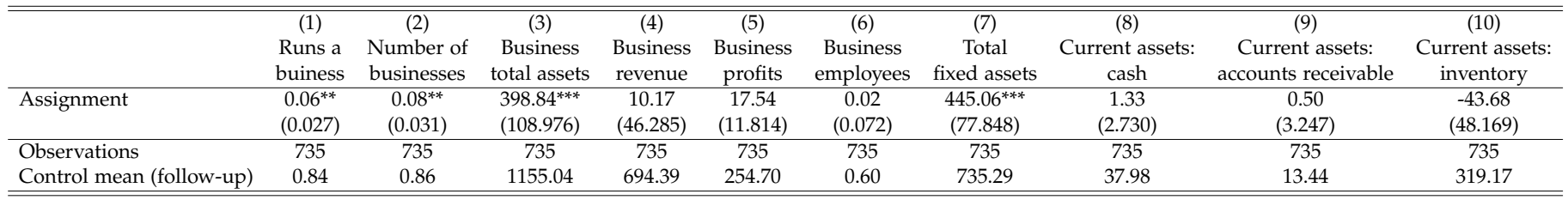

PANEL C: 12-MONTH FOLLOW-UP

\begin{tabular}{|c|c|c|c|c|c|c|c|c|c|c|}
\hline & $\begin{array}{c}(1) \\
\text { Runs a } \\
\text { buiness }\end{array}$ & $\begin{array}{c}\text { (2) } \\
\text { Number of } \\
\text { businesses }\end{array}$ & $\begin{array}{c}\text { (3) } \\
\text { Business } \\
\text { total assets }\end{array}$ & $\begin{array}{l}\text { (4) } \\
\text { Business } \\
\text { revenue }\end{array}$ & $\begin{array}{c}\text { (5) } \\
\text { Business } \\
\text { profits }\end{array}$ & $\begin{array}{c}\text { (6) } \\
\text { Business } \\
\text { employees }\end{array}$ & $\begin{array}{c}(7) \\
\text { Total } \\
\text { fixed assets }\end{array}$ & $\begin{array}{c}(8) \\
\text { Current assets: } \\
\text { cash }\end{array}$ & $\begin{array}{c}(9) \\
\text { Current assets: } \\
\text { accounts receivable }\end{array}$ & $\begin{array}{c}\text { (10) } \\
\text { Current assets: } \\
\text { inventory }\end{array}$ \\
\hline Assignment & $\begin{array}{l}0.10^{* * *} \\
(0.030)\end{array}$ & $\begin{array}{l}0.09^{* * *} \\
(0.029)\end{array}$ & $\begin{array}{l}465.72^{* * *} \\
(112.464)\end{array}$ & $\begin{array}{c}-18.32 \\
(48.501)\end{array}$ & $\begin{array}{l}32.43^{* * *} \\
(12.479)\end{array}$ & $\begin{array}{c}0.01 \\
(0.074)\end{array}$ & $\begin{array}{c}503.90^{* * *} \\
(80.790)\end{array}$ & $\begin{array}{c}1.17 \\
(1.968)\end{array}$ & $\begin{array}{c}-4.64 \\
(3.245)\end{array}$ & $\begin{array}{c}-28.64 \\
(41.649)\end{array}$ \\
\hline Observations & 720 & 720 & 720 & 720 & 720 & 720 & 720 & 720 & 720 & 720 \\
\hline Control mean (follow-up) & 0.79 & 0.81 & 982.49 & 720.03 & 253.13 & 0.58 & 640.63 & 28.10 & 14.78 & 244.01 \\
\hline
\end{tabular}

PANEL D: 18-MONTH FOLLOW-UP

\begin{tabular}{|c|c|c|c|c|c|c|c|c|c|c|}
\hline & (1) & (2) & (3) & (4) & (5) & (6) & (7) & (8) & (9) & (10) \\
\hline & $\begin{array}{l}\text { Runs a } \\
\text { buiness }\end{array}$ & $\begin{array}{l}\text { Number of } \\
\text { businesses }\end{array}$ & $\begin{array}{l}\text { Business } \\
\text { total assets }\end{array}$ & $\begin{array}{l}\text { Business } \\
\text { revenue }\end{array}$ & $\begin{array}{c}\text { Business } \\
\text { profits }\end{array}$ & $\begin{array}{l}\text { Business } \\
\text { employees }\end{array}$ & $\begin{array}{c}\text { Total } \\
\text { fixed assets }\end{array}$ & $\begin{array}{c}\text { Current assets: } \\
\text { cash }\end{array}$ & $\begin{array}{l}\text { Current assets: } \\
\text { accounts receivable }\end{array}$ & $\begin{array}{l}\text { Current assets: } \\
\text { inventory }\end{array}$ \\
\hline Assignment & $\begin{array}{l}0.11^{* * *} \\
(0.031)\end{array}$ & $\begin{array}{l}0.10^{* * *} \\
(0.031)\end{array}$ & $\begin{array}{l}366.86^{* * *} \\
(108.238)\end{array}$ & $\begin{array}{c}16.25 \\
(47.979)\end{array}$ & $\begin{array}{l}34.82^{* * *} \\
(12.187)\end{array}$ & $\begin{array}{c}0.07 \\
(0.074)\end{array}$ & $\begin{array}{c}402.23^{* * *} \\
(85.846)\end{array}$ & $\begin{array}{c}4.57^{* *} \\
(1.949)\end{array}$ & $\begin{array}{c}0.82 \\
(0.929)\end{array}$ & $\begin{array}{c}-26.42 \\
(36.308)\end{array}$ \\
\hline Observations & 710 & 710 & 710 & 710 & 710 & 710 & 710 & 710 & 710 & 710 \\
\hline Control mean (follow-up) & 0.77 & 0.78 & 914.67 & 691.46 & 250.81 & 0.53 & 635.36 & 23.85 & 2.14 & 200.32 \\
\hline
\end{tabular}

PANEL D: 24-MONTH FOLLOW-UP

\begin{tabular}{|c|c|c|c|c|c|c|c|c|c|c|}
\hline & (1) & (2) & (3) & (4) & (5) & (6) & (7) & (8) & (9) & (10) \\
\hline & $\begin{array}{l}\text { Runs a } \\
\text { buiness }\end{array}$ & $\begin{array}{l}\text { Number of } \\
\text { businesses }\end{array}$ & $\begin{array}{l}\text { Business } \\
\text { total assets }\end{array}$ & $\begin{array}{l}\text { Business } \\
\text { revenue }\end{array}$ & $\begin{array}{c}\text { Business } \\
\text { profits }\end{array}$ & $\begin{array}{l}\text { Business } \\
\text { employees }\end{array}$ & $\begin{array}{c}\text { Total } \\
\text { fixed assets }\end{array}$ & $\begin{array}{l}\text { Current assets: } \\
\text { cash }\end{array}$ & $\begin{array}{l}\text { Current assets: } \\
\text { accounts receivable }\end{array}$ & $\begin{array}{c}\text { Current assets: } \\
\text { inventory }\end{array}$ \\
\hline Assignment & $\begin{array}{l}0.11^{* * *} \\
(0.033)\end{array}$ & $\begin{array}{l}0.10^{* * *} \\
(0.033)\end{array}$ & $\begin{array}{c}329.26^{* * *} \\
(96.642)\end{array}$ & $\begin{array}{c}7.38 \\
(46.161)\end{array}$ & $\begin{array}{l}37.14^{* * *} \\
(12.809)\end{array}$ & $\begin{array}{c}0.09 \\
(0.062)\end{array}$ & $\begin{array}{c}377.49^{* * *} \\
(78.869)\end{array}$ & $\begin{array}{l}3.30^{* *} \\
(1.501)\end{array}$ & $\begin{array}{c}-0.02 \\
(0.178)\end{array}$ & $\begin{array}{c}-51.52 \\
(36.312)\end{array}$ \\
\hline Observations & 696 & 696 & 696 & 696 & 696 & 696 & 696 & 696 & 696 & 696 \\
\hline Control mean (follow-up) & 0.74 & 0.75 & 805.69 & 667.82 & 249.81 & 0.46 & 574.72 & 22.06 & 0.50 & 183.10 \\
\hline
\end{tabular}


Table A.35: Disaggregating results by survey wave: other outcomes

PANEL A: THREE-MONTH FOLLOW-UP

\begin{tabular}{|c|c|c|c|c|c|c|c|c|}
\hline & $\begin{array}{c}(1) \\
\text { Household } \\
\text { income }\end{array}$ & $\begin{array}{c}(2) \\
\text { Household consumption } \\
\text { expenditure }\end{array}$ & $\begin{array}{l}\text { (3) } \\
\text { Household } \\
\text { savings }\end{array}$ & $\begin{array}{c}(4) \\
\text { Household } \\
\text { loans }\end{array}$ & $\begin{array}{c}(5) \\
\text { Has a } \\
\text { wage job }\end{array}$ & $\begin{array}{c}\text { (6) } \\
\text { Number of } \\
\text { wage jobs }\end{array}$ & $\begin{array}{c}(7) \\
\text { Total } \\
\text { wage hours }\end{array}$ & $\begin{array}{c}(8) \\
\text { Total } \\
\text { wage income }\end{array}$ \\
\hline Assignment & $\begin{array}{c}13.96 \\
(13.898)\end{array}$ & $\begin{array}{l}10.12^{*} \\
(5.318)\end{array}$ & $\begin{array}{c}28.66 \\
(35.259)\end{array}$ & $\begin{array}{c}-38.82^{* * *} \\
(8.918)\end{array}$ & $\begin{array}{l}-0.06^{*} \\
(0.030)\end{array}$ & $\begin{array}{l}-0.06^{*} \\
(0.030)\end{array}$ & $\begin{array}{l}-2.76^{*} \\
(1.519)\end{array}$ & $\begin{array}{l}-10.22 \\
(6.329)\end{array}$ \\
\hline Observations & 737 & 737 & 737 & 737 & 737 & 737 & 737 & 737 \\
\hline Control mean (follow-up) & 335.68 & 203.66 & 198.56 & 87.26 & 0.23 & 0.23 & 11.19 & 44.01 \\
\hline
\end{tabular}

PANEL B: SIX-MONTH FOLLOW-UP

\begin{tabular}{|c|c|c|c|c|c|c|c|c|}
\hline & $\begin{array}{c}\text { (1) } \\
\text { Household } \\
\text { income }\end{array}$ & $\begin{array}{l}\text { (2) } \\
\text { Household consumption } \\
\text { expenditure }\end{array}$ & $\begin{array}{l}\text { (3) } \\
\text { Household } \\
\text { savings }\end{array}$ & $\begin{array}{l}(4) \\
\text { Household } \\
\text { loans }\end{array}$ & $\begin{array}{c}\text { (5) } \\
\text { Has a } \\
\text { wage job }\end{array}$ & $\begin{array}{c}\text { (6) } \\
\text { Number of } \\
\text { wage jobs }\end{array}$ & $\begin{array}{c}\text { (7) } \\
\text { Total } \\
\text { wage hours }\end{array}$ & $\begin{array}{c}(8) \\
\text { Total } \\
\text { wage income }\end{array}$ \\
\hline Assignment & $\begin{array}{c}14.85 \\
(15.494)\end{array}$ & $\begin{array}{c}14.52^{* * *} \\
(5.427)\end{array}$ & $\begin{array}{c}-9.18 \\
(29.566)\end{array}$ & $\begin{array}{c}-39.05^{* * *} \\
(7.169)\end{array}$ & $\begin{array}{c}-0.05 \\
(0.030)\end{array}$ & $\begin{array}{c}-0.04 \\
(0.030)\end{array}$ & $\begin{array}{l}-2.66^{*} \\
(1.509)\end{array}$ & $\begin{array}{l}-10.56 \\
(6.852)\end{array}$ \\
\hline Observations & 735 & 735 & 735 & 735 & 735 & 735 & 735 & 735 \\
\hline Control mean (follow-up) & 362.83 & 212.64 & 167.68 & 75.65 & 0.23 & 0.22 & 11.32 & 48.27 \\
\hline
\end{tabular}

PANEL C: 12-MONTH FOLLOW-UP

\begin{tabular}{|c|c|c|c|c|c|c|c|c|}
\hline & $\begin{array}{c}1(1) \\
\text { Household } \\
\text { income }\end{array}$ & $\begin{array}{c}(2) \\
\text { Household consumption } \\
\text { expenditure }\end{array}$ & $\begin{array}{c}(3) \\
\text { Household } \\
\text { savings }\end{array}$ & $\begin{array}{c}(4) \\
\text { Household } \\
\text { loans }\end{array}$ & $\begin{array}{c}(5) \\
\text { Has a } \\
\text { wage job }\end{array}$ & $\begin{array}{c}(6) \\
\text { Number of } \\
\text { wage jobs }\end{array}$ & $\begin{array}{c}(7) \\
\text { Total } \\
\text { wage hours }\end{array}$ & $\begin{array}{c}(8) \\
\text { Total } \\
\text { wage income }\end{array}$ \\
\hline Assignment & $\begin{array}{l}37.21^{* *} \\
(16.303)\end{array}$ & $\begin{array}{l}11.74^{* *} \\
(5.223)\end{array}$ & $\begin{array}{c}30.50 \\
(28.450)\end{array}$ & $\begin{array}{c}-20.59^{* * *} \\
(4.942)\end{array}$ & $\begin{array}{l}-0.07^{* *} \\
(0.032)\end{array}$ & $\begin{array}{l}-0.07^{* *} \\
(0.032)\end{array}$ & $\begin{array}{l}-3.97^{* *} \\
(1.598)\end{array}$ & $\begin{array}{c}-14.93^{* *} \\
(7.362)\end{array}$ \\
\hline Observations & 720 & 720 & 720 & 720 & 720 & 720 & 720 & 720 \\
\hline Control mean (follow-up) & 367.45 & 217.68 & 107.20 & 39.57 & 0.25 & 0.25 & 12.33 & 53.36 \\
\hline
\end{tabular}

PANEL D: 18-MONTH FOLLOW-UP

\begin{tabular}{|c|c|c|c|c|c|c|c|c|}
\hline & $\begin{array}{l}(1) \\
\text { Household } \\
\text { income }\end{array}$ & $\begin{array}{c}(2) \\
\text { Household consumption } \\
\text { expenditure }\end{array}$ & $\begin{array}{l}(3) \\
\text { Household } \\
\text { savings }\end{array}$ & $\begin{array}{l}(4) \\
\text { Household } \\
\text { loans }\end{array}$ & $\begin{array}{c}(5) \\
\text { Has a } \\
\text { wage job }\end{array}$ & $\begin{array}{l}\text { (6) } \\
\text { Number of } \\
\text { wage jobs }\end{array}$ & $\begin{array}{c}\text { (7) } \\
\text { Total } \\
\text { wage hours }\end{array}$ & $\begin{array}{c}(8) \\
\text { Total } \\
\text { wage income }\end{array}$ \\
\hline Assignment & $\begin{array}{l}44.01^{* * *} \\
(16.667)\end{array}$ & $\begin{array}{c}17.87^{* * *} \\
(4.907)\end{array}$ & $\begin{array}{c}23.55 \\
(22.349)\end{array}$ & $\begin{array}{c}-10.94^{* * *} \\
(3.578)\end{array}$ & $\begin{array}{l}-0.08^{* *} \\
(0.031)\end{array}$ & $\begin{array}{l}-0.08^{* *} \\
(0.031)\end{array}$ & $\begin{array}{l}-4.72^{* * *} \\
(1.612)\end{array}$ & $\begin{array}{c}-20.38^{* * *} \\
(7.664)\end{array}$ \\
\hline Observations & 710 & 710 & 710 & 710 & 710 & 710 & 710 & 710 \\
\hline Control mean (follow-up) & 359.29 & 232.94 & 60.91 & 18.10 & 0.26 & 0.26 & 13.36 & 60.33 \\
\hline \multicolumn{9}{|c|}{ PANEL D: 24-MONTH FOLLOW-UP } \\
\hline & $\begin{array}{c}\text { (1) } \\
\text { Household } \\
\text { income }\end{array}$ & $\begin{array}{c}\text { (2) } \\
\text { Household consumption } \\
\text { expenditure }\end{array}$ & $\begin{array}{c}\text { (3) } \\
\text { Household } \\
\text { savings }\end{array}$ & $\begin{array}{c}(4) \\
\text { Household } \\
\text { loans }\end{array}$ & $\begin{array}{c}\text { (5) } \\
\text { Has a } \\
\text { wage job }\end{array}$ & $\begin{array}{c}\text { (6) } \\
\text { Number of } \\
\text { wage jobs }\end{array}$ & $\begin{array}{c}\text { (7) } \\
\text { Total } \\
\text { wage hours } \\
\end{array}$ & $\begin{array}{c}(8) \\
\text { Total } \\
\text { wage income }\end{array}$ \\
\hline Assignment & $\begin{array}{l}51.01^{* * *} \\
(18.156)\end{array}$ & $\begin{array}{c}9.52^{*} \\
(5.016) \\
\end{array}$ & $\begin{array}{c}8.99 \\
(7.790) \\
\end{array}$ & $\begin{array}{l}-3.72^{*} \\
(1.922)\end{array}$ & $\begin{array}{l}-0.07^{* *} \\
(0.033)\end{array}$ & $\begin{array}{l}-0.07^{* *} \\
(0.033)\end{array}$ & $\begin{array}{l}-4.10^{* *} \\
(1.683)\end{array}$ & $\begin{array}{c}-20.39^{* *} \\
(8.835)\end{array}$ \\
\hline Observations & 696 & 696 & 696 & 696 & 696 & 696 & 696 & 696 \\
\hline Control mean (follow-up) & 361.96 & 235.89 & 26.24 & 7.45 & 0.28 & 0.28 & 14.30 & 71.59 \\
\hline
\end{tabular}


Figure A.4: Empirical CDFs for total fixed assets, disaggregated by survey wave

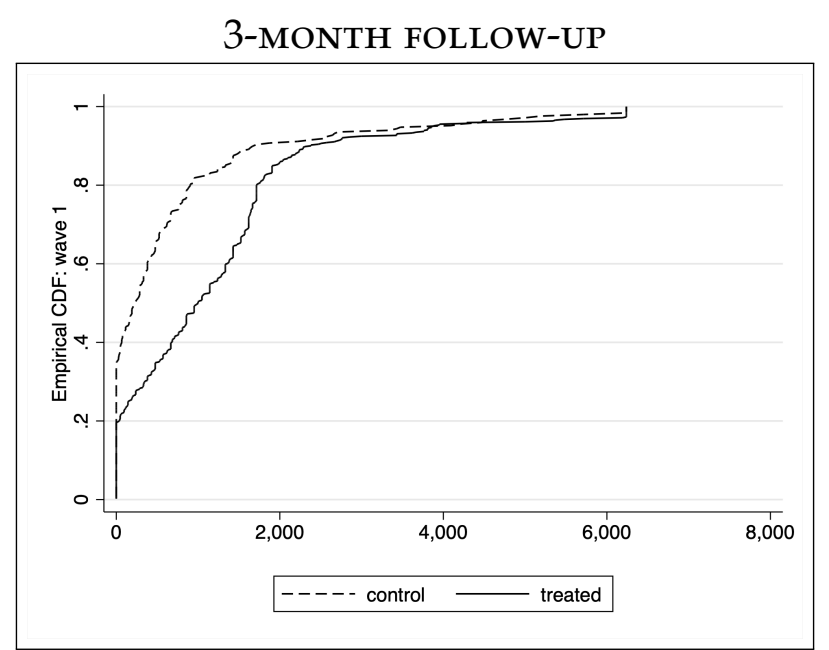

12-MONTH FOLLOW-UP

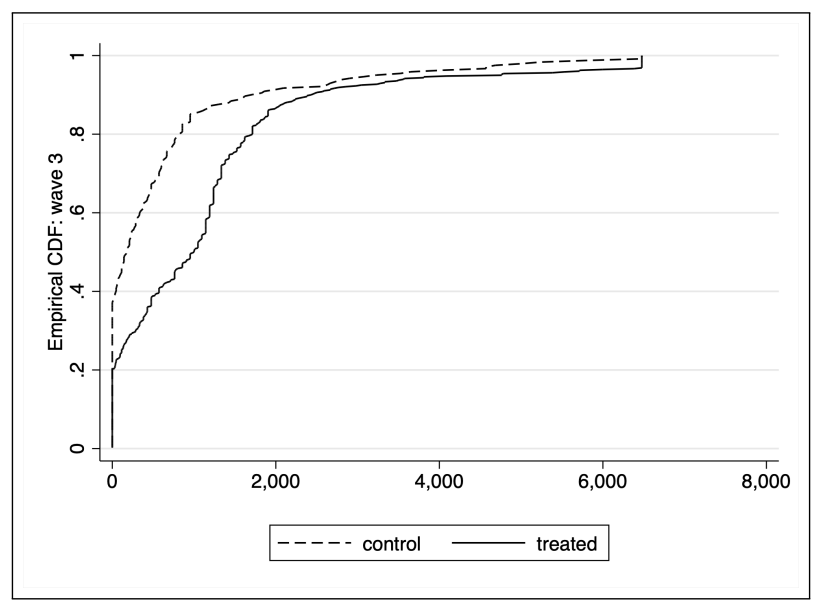

24-MONTH FOLLOW-UP

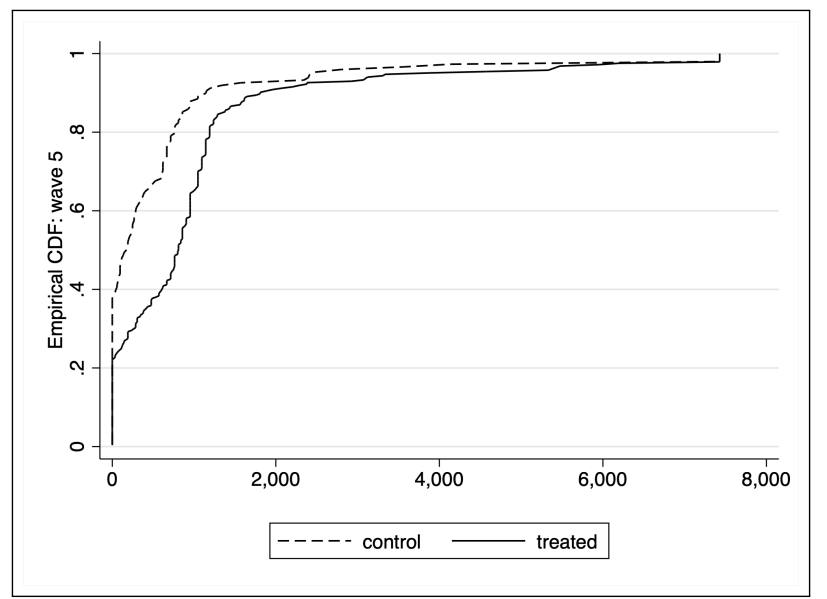

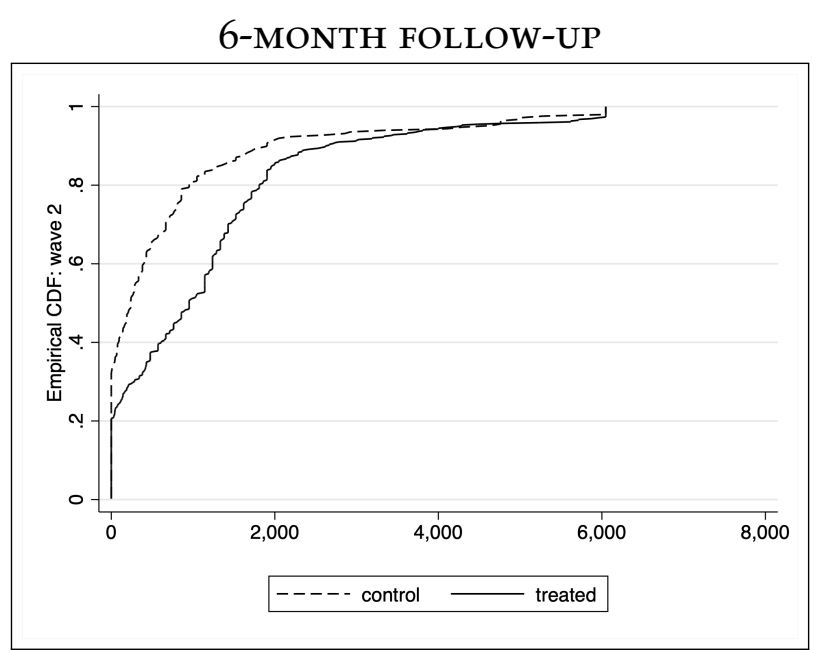

18-MONTH FOLLOW-UP

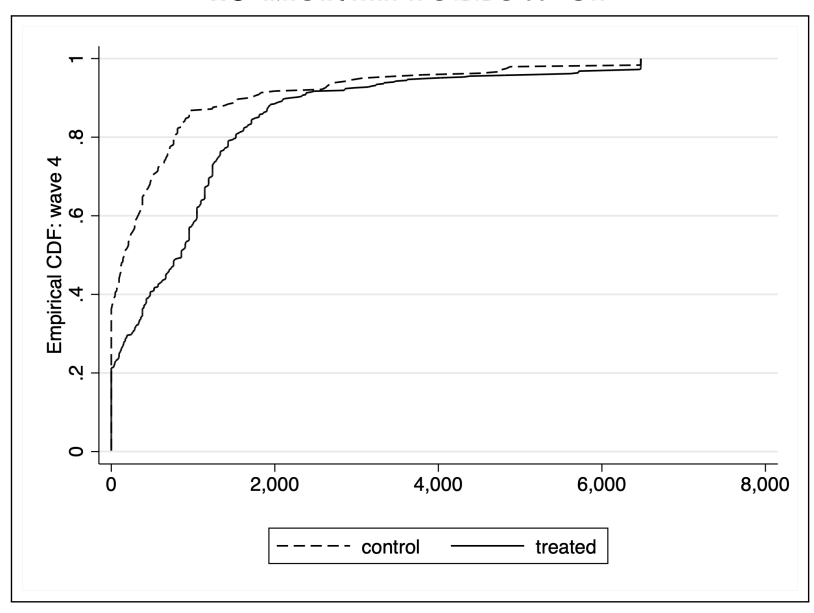


Figure A.5: Empirical CDFs for business profits, disaggregated by survey wave

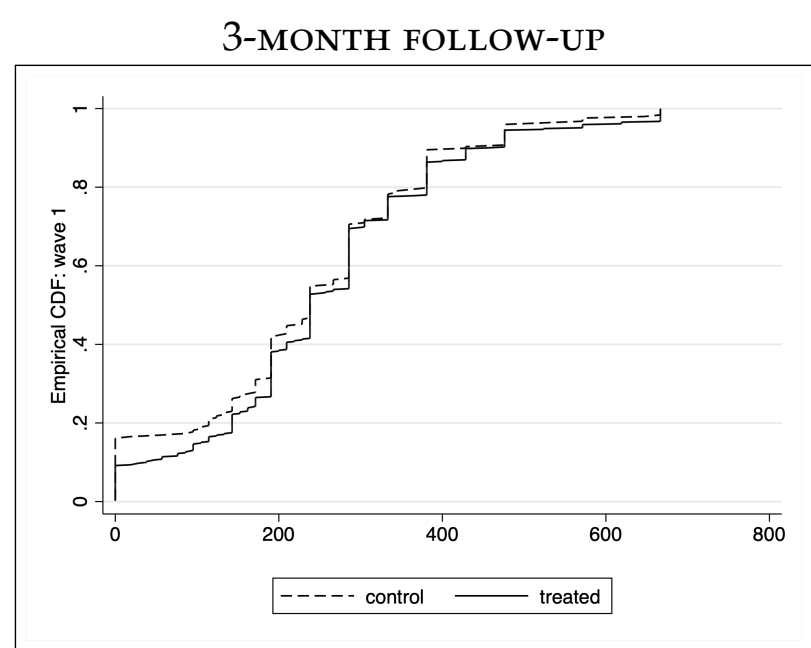

12-MONTH FOLLOW-UP

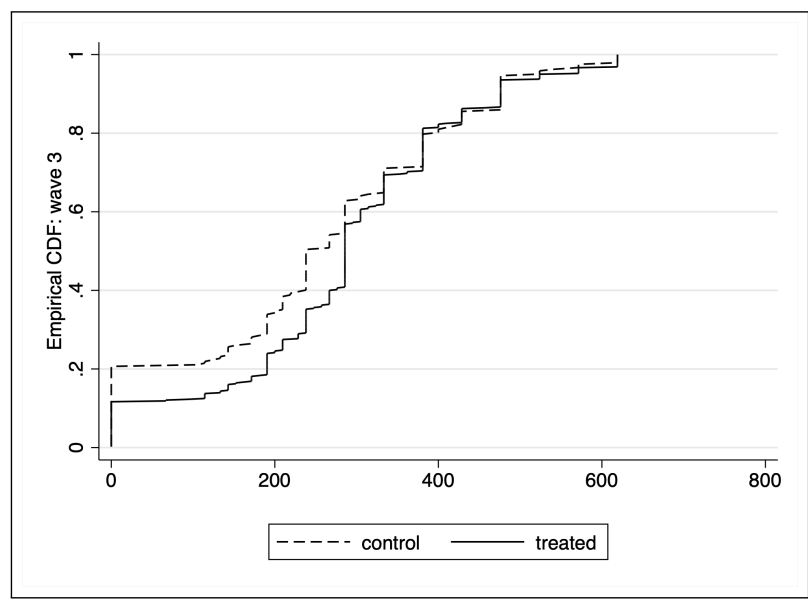

24-MONTH FOLLOW-UP

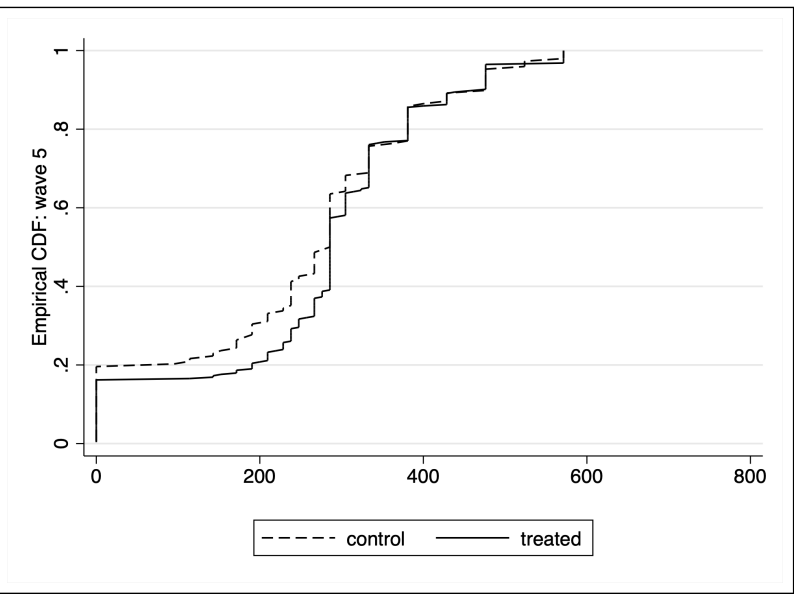

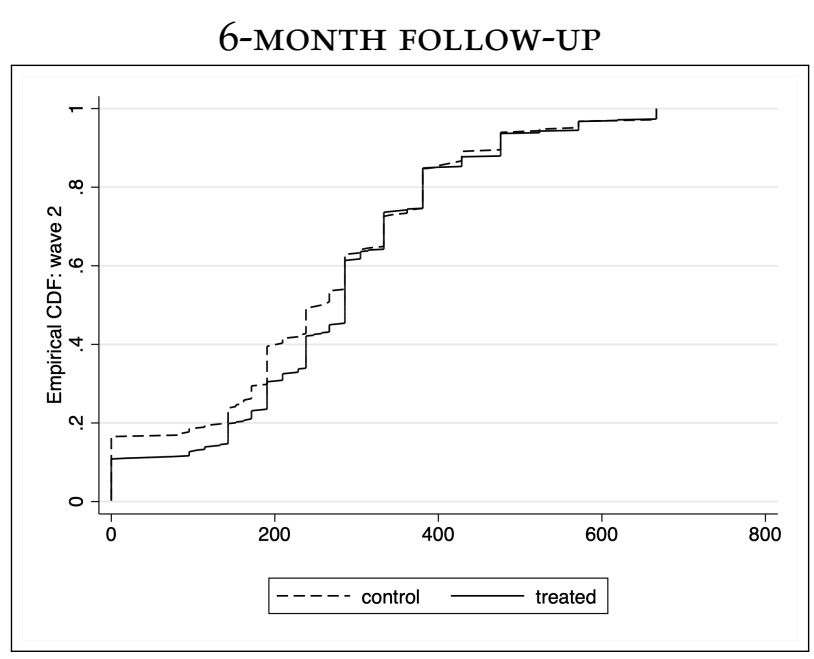

18-MONTH FOLLOW-UP

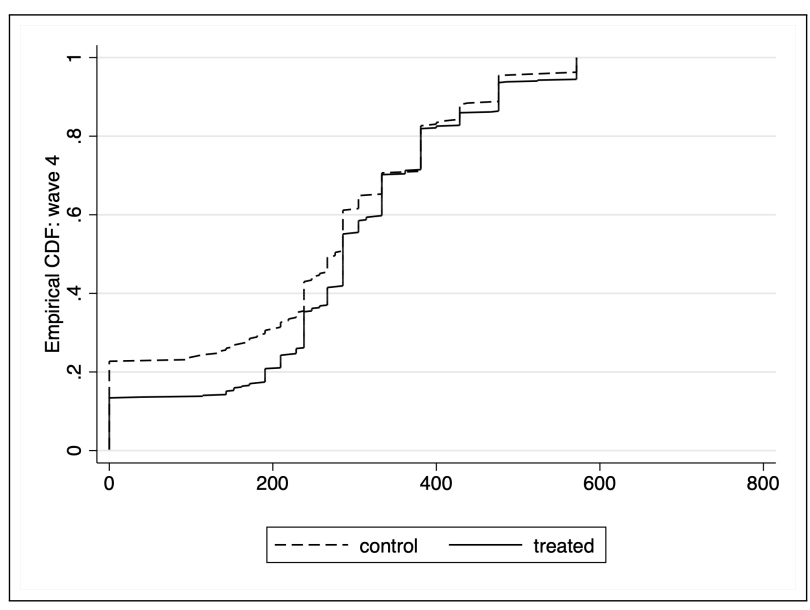


Figure A.6: Empirical CDFs for household consumption, disaggregated by survey wave

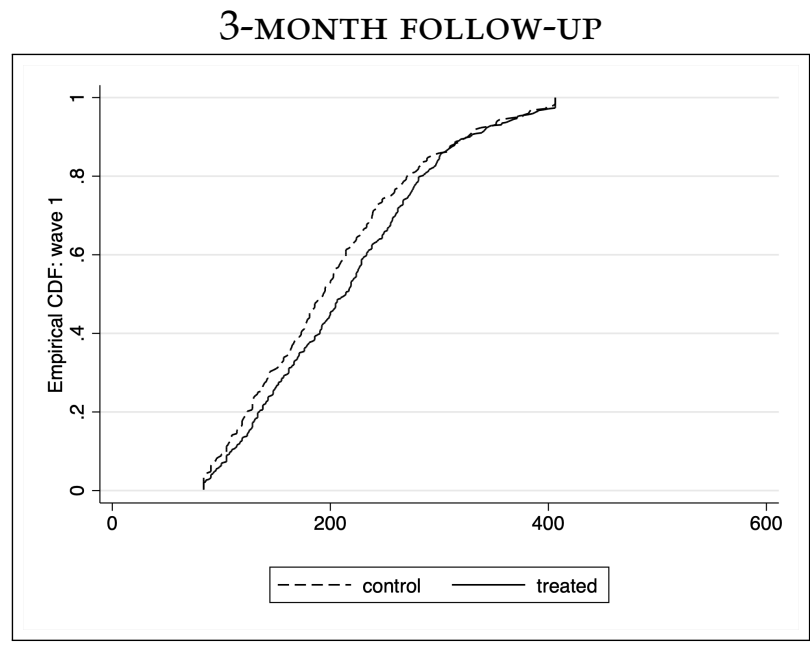

12-MONTH FOLLOW-UP

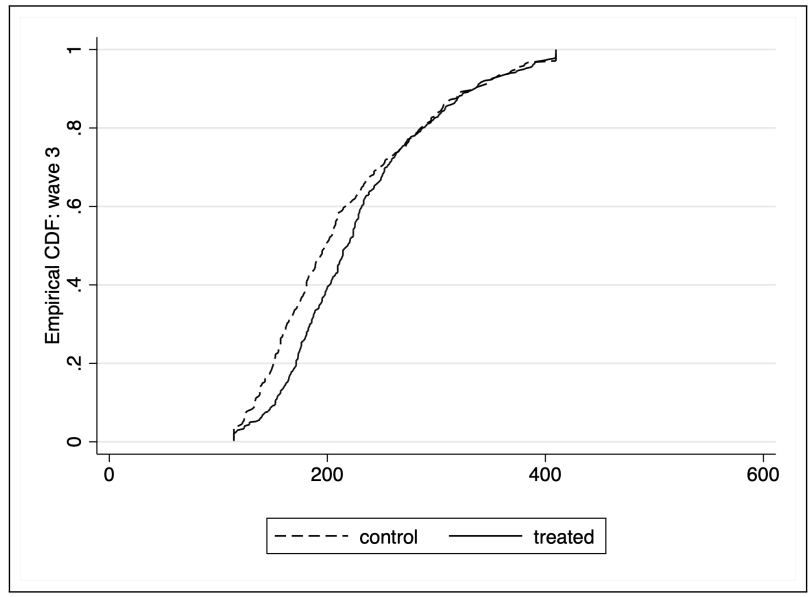

24-MONTH FOLLOW-UP

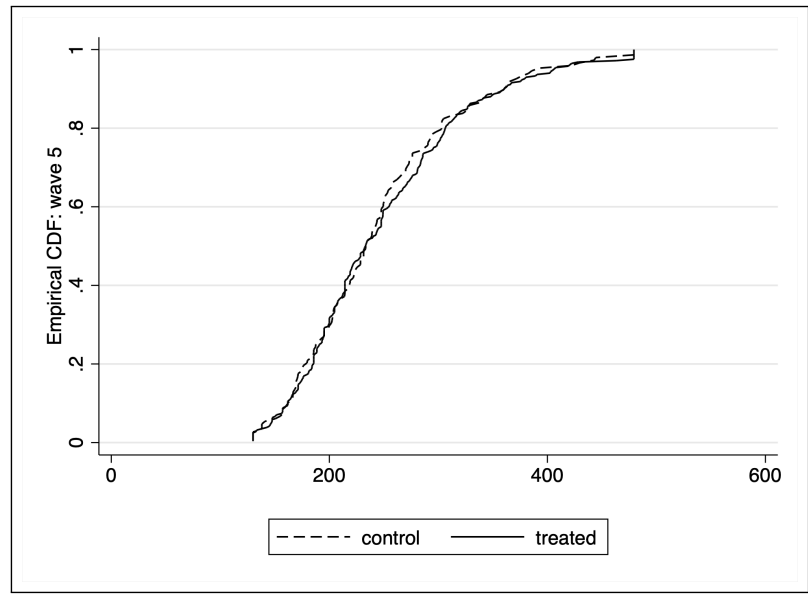

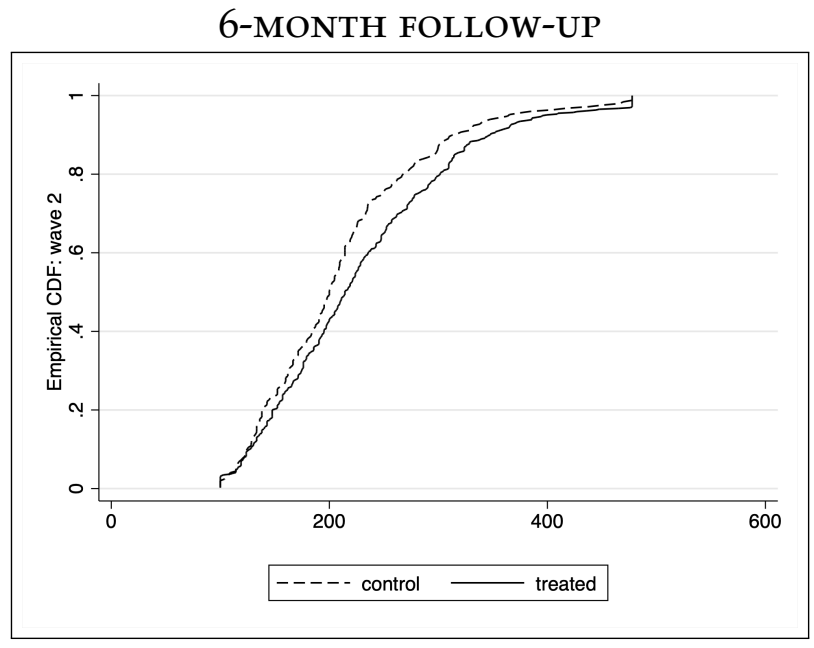

18-MONTH FOLLOW-UP

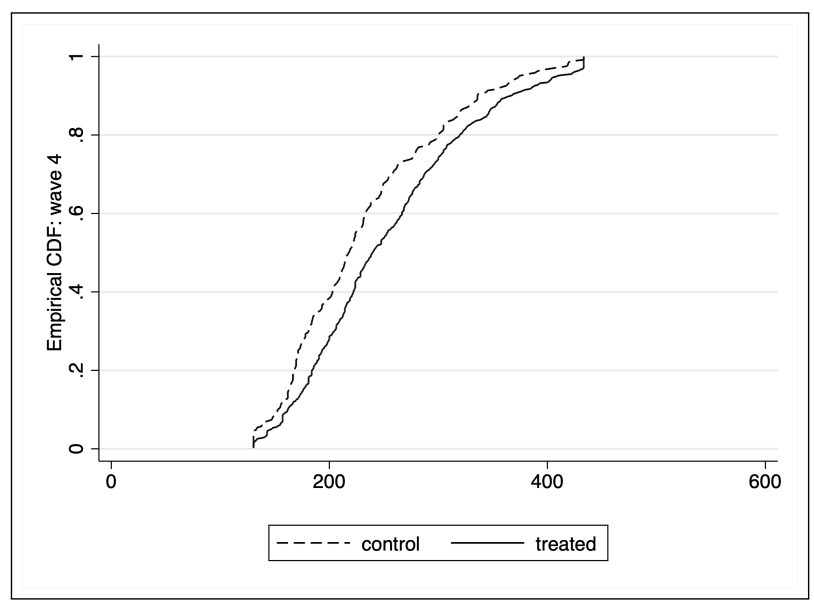


Figure A.7: Empirical CDFs for schooling expenditure, disaggregated by survey wave

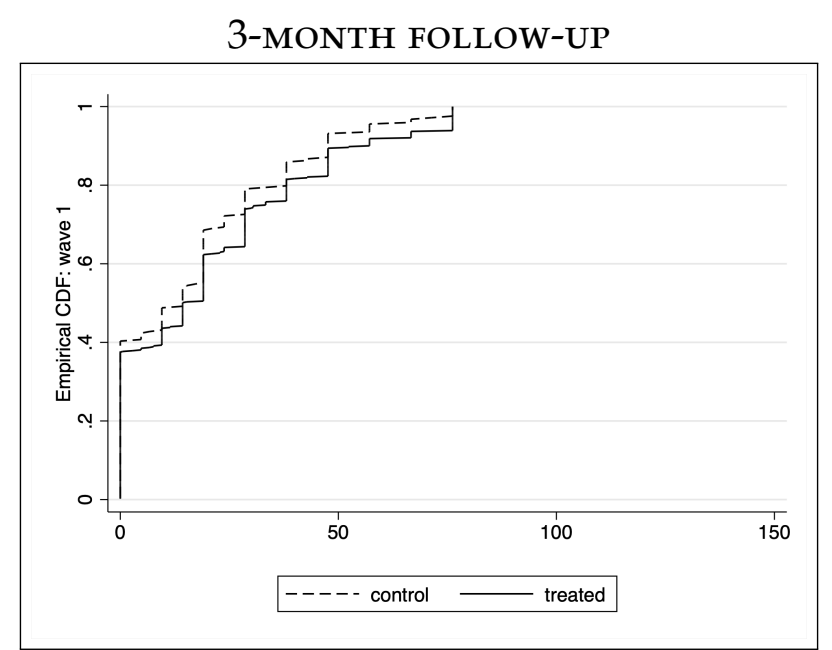

12-MONTH FOLLOW-UP

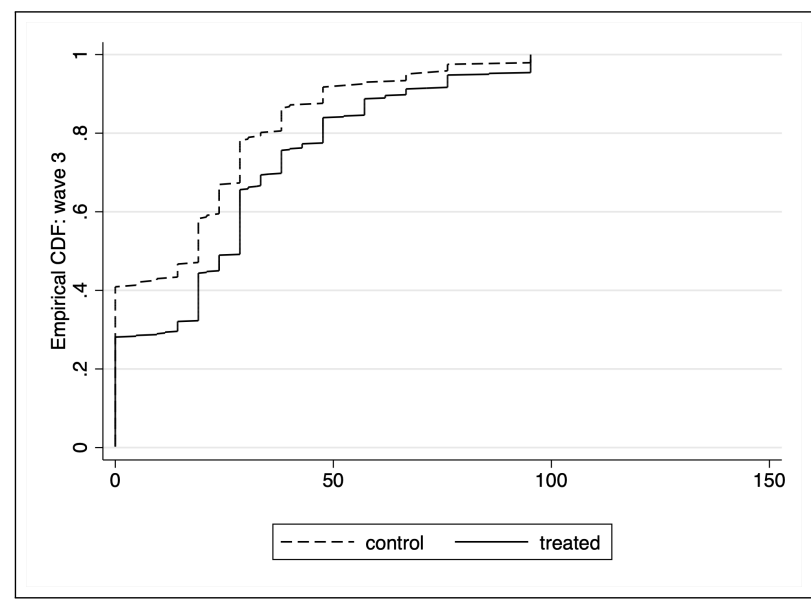

24-MONTH FOLLOW-UP

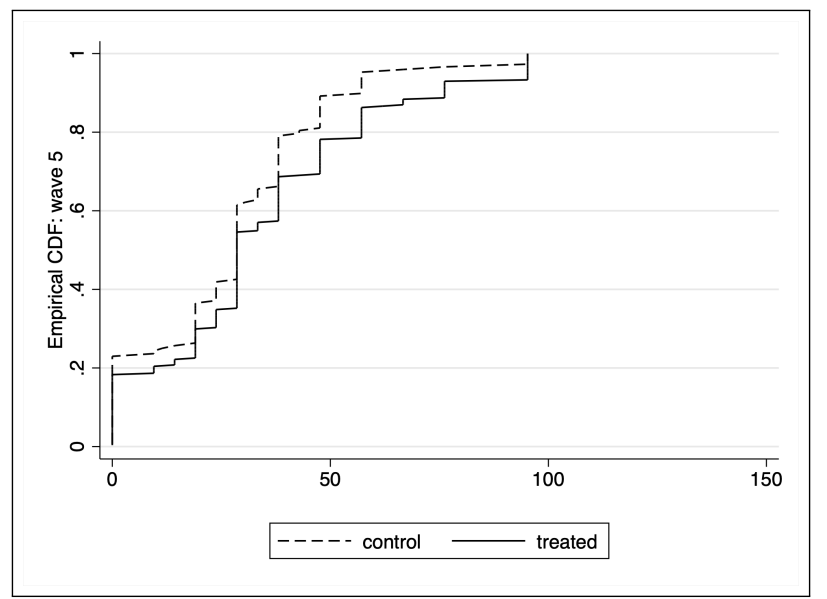

6-MONTH FOLLOW-UP

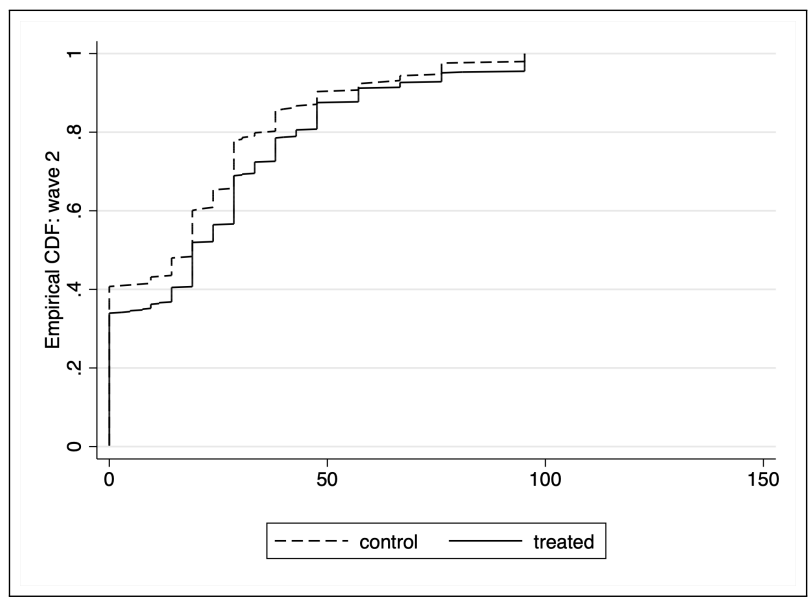

18-MONTH FOLLOW-UP

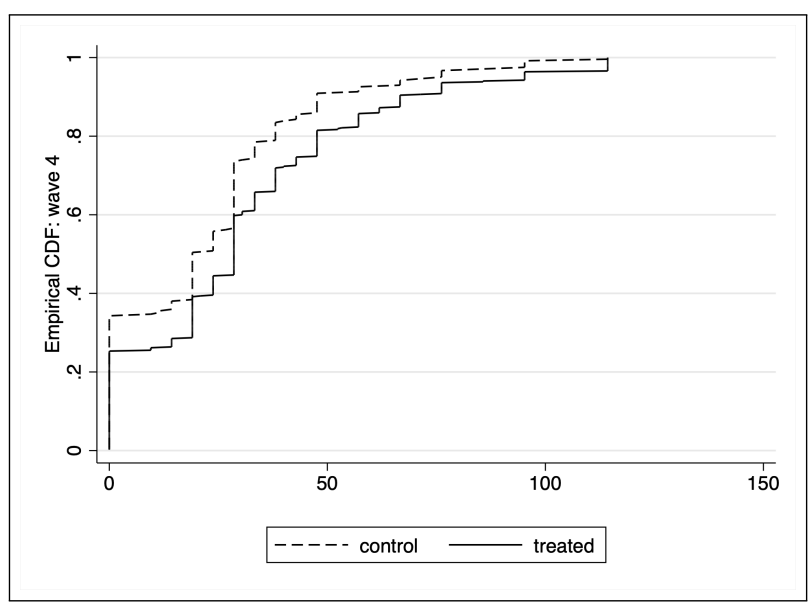




\section{K Robustness to outliers}

Here we consider outliers: we take the main treatment effects of interest from our previous analysis, and subject them to increasing degrees of winsorization.

Table A.36: Robustness of main results to Winsorizing

Panel A: Winsorizing $2.5 \%$ top AND bottom (estimates IN THE MAIN PAPER)

\begin{tabular}{lcccccccccc}
\hline & $\begin{array}{c}(1) \\
\text { Number of } \\
\text { businesses }\end{array}$ & $\begin{array}{c}\text { Business } \\
\text { assets }\end{array}$ & $\begin{array}{c}\text { Fixed } \\
\text { assets }\end{array}$ & $\begin{array}{c}(4) \\
\text { Business } \\
\text { revenue }\end{array}$ & $\begin{array}{c}(5) \\
\text { Business } \\
\text { profits }\end{array}$ & $\begin{array}{c}(6) \\
\text { Household } \\
\text { loans }\end{array}$ & $\begin{array}{c}(7) \\
\text { Household } \\
\text { consumption }\end{array}$ & $\begin{array}{c}(8) \\
\text { Expenditure: } \\
\text { schooling }\end{array}$ & $\begin{array}{c}\text { Expenditure: } \\
\text { food }\end{array}$ \\
\hline Assignment & $0.10^{* * *}$ & $401.22^{* * *}$ & $438.05^{* * *}$ & 1.82 & $26.93^{* * *}$ & $-22.81^{* * *}$ & $12.95^{* * *}$ & $5.70^{* * *}$ & $2.61^{* * *}$ \\
& $(0.025)$ & $(89.940)$ & $(67.147)$ & $(39.654)$ & $(9.929)$ & $(3.653)$ & $(3.374)$ & $(1.297)$ & $(0.900)$ \\
\hline Control mean & 0.82 & 1003.34 & 660.19 & 689.65 & 249.31 & 46.05 & 220.40 & 22.05 & 52.80 \\
Effect size (\%) & 11.8 & 40.0 & 66.4 & 0.3 & 10.8 & -49.5 & 5.9 & 25.8 & 4.9 \\
Observations & 3608 & 3608 & 3608 & 3608 & 3608 & 3608 & 3608 & 3608 & 3608 \\
\hline
\end{tabular}

Panel B: Winsorizing $1 \%$ top AND Bottom

\begin{tabular}{lccccccccc}
\hline & $\begin{array}{c}(1) \\
\text { Number of } \\
\text { businesses }\end{array}$ & $\begin{array}{c}(2) \\
\text { Business } \\
\text { assets }\end{array}$ & $\begin{array}{c}\text { Fixed } \\
\text { assets }\end{array}$ & $\begin{array}{c}(4) \\
\text { Business } \\
\text { revenue }\end{array}$ & $\begin{array}{c}(5) \\
\text { Business } \\
\text { profits }\end{array}$ & $\begin{array}{c}(6) \\
\text { Household } \\
\text { loans }\end{array}$ & $\begin{array}{c}\text { Household } \\
\text { consumption }\end{array}$ & $\begin{array}{c}\text { Expenditure: } \\
\text { schooling }\end{array}$ & $\begin{array}{c}\text { Expenditure: } \\
\text { food }\end{array}$ \\
\hline Assignment & $0.10^{* * *}$ & $420.07^{* * *}$ & $462.09^{* * *}$ & -7.25 & $29.03^{* * *}$ & $-20.70^{* * *}$ & $13.27^{* * * *}$ & $6.28^{* * * *}$ & $2.63^{* * *}$ \\
& $(0.025)$ & $(100.763)$ & $(77.668)$ & $(47.442)$ & $(10.442)$ & $(4.107)$ & $(3.622)$ & $(1.410)$ & $(0.936)$ \\
\hline Control mean & 0.82 & 1042.31 & 698.94 & 716.96 & 251.60 & 48.33 & 221.65 & 22.33 & 52.94 \\
Effect size (\%) & 12.2 & 40.3 & 66.1 & -1.0 & 11.5 & -42.8 & 6.0 & 28.1 & 5.0 \\
Observations & 3608 & 3608 & 3608 & 3608 & 3608 & 3608 & 3608 & 3608 & 3608 \\
\hline
\end{tabular}

Panel C: Winsorizing 5\% TOP AND Bottom

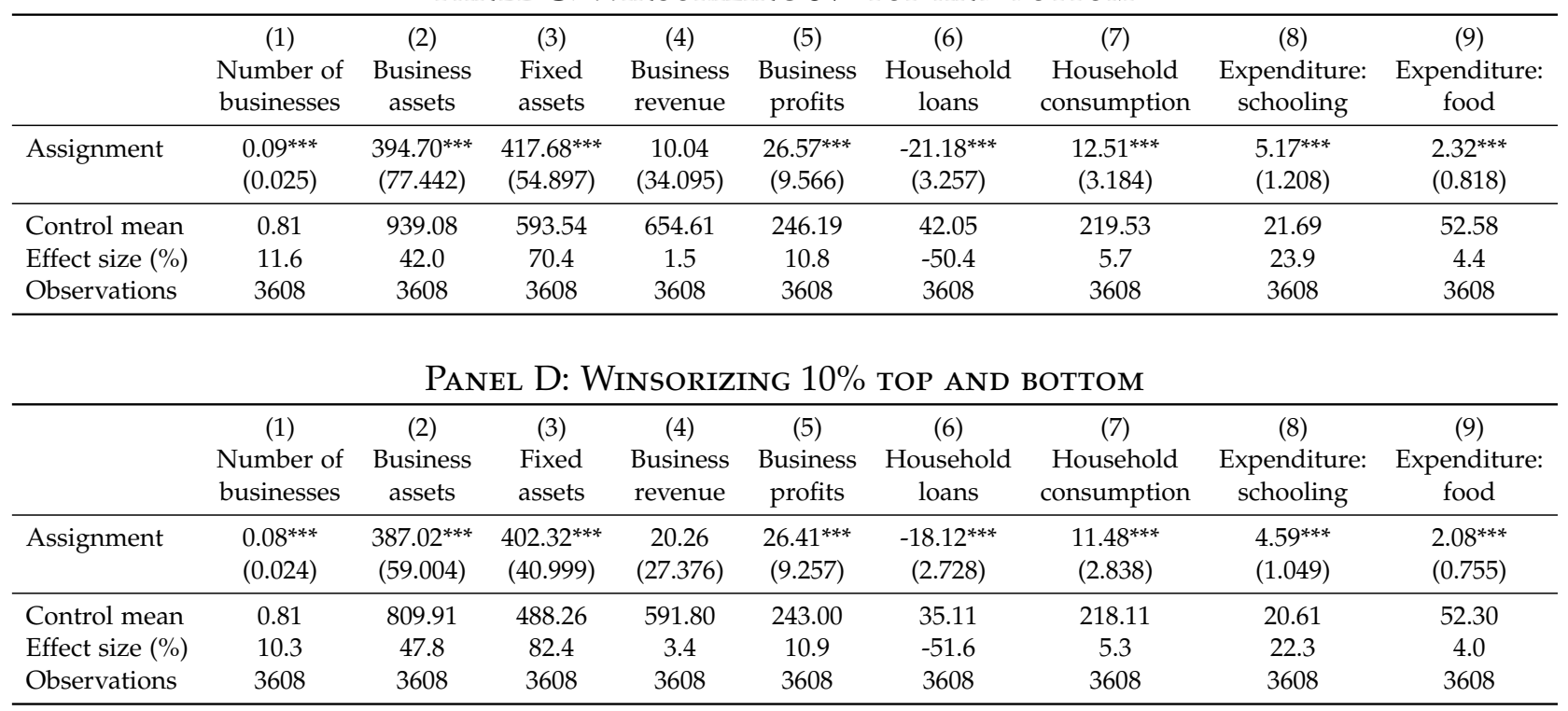




\section{Local Average Treatment Effect estimations}

\begin{tabular}{lcccccc}
\hline & $\begin{array}{c}(1) \\
\text { Runs a } \\
\text { buiness }\end{array}$ & $\begin{array}{c}\text { Number of } \\
\text { businesses }\end{array}$ & $\begin{array}{c}(3) \\
\text { Business } \\
\text { total assets }\end{array}$ & $\begin{array}{c}(4) \\
\text { Business } \\
\text { revenue }\end{array}$ & $\begin{array}{c}(5) \\
\text { Business } \\
\text { profits }\end{array}$ & $\begin{array}{c}(6) \\
\text { Business } \\
\text { employees }\end{array}$ \\
\hline Take-up & 0.16 & 0.17 & 726.21 & 3.29 & 49.06 & 0.06 \\
& $(0.04)$ & $(0.04)$ & $(159.93)$ & $(71.76)$ & $(17.94)$ & $(0.10)$ \\
& {$[0.00]^{* * *}$} & {$[0.00]^{* * *}$} & {$[0.00]^{* * *}$} & {$[0.96]$} & {$[0.01]^{* * *}$} & {$[0.54]$} \\
& $\{0.00\}^{* * *}$ & $\{0.00\}^{* * *}$ & $\{0.00\}^{* * *}$ & $\{0.47\}$ & $\{0.00\}^{* * *}$ & $\{0.28\}$ \\
\hline Control mean (follow-up) & 0.80 & 0.82 & 1003.34 & 689.65 & 249.31 & 0.56 \\
Observations & 3,608 & 3,608 & 3,608 & 3,608 & 3,608 & 3,608 \\
\hline
\end{tabular}

Note: In this table we report the LATE estimates of the combined treatment on primary outcomes, obtained by least-squares estimation. Below each coefficient, we report a standard error in parenthesis, a $p$-value in brackets, and a $q$-value in curly braces. Standard errors allow for clustering at the level of the individual. $q$-values are obtained using the sharpened procedure of (Benjamini et al., 2006). We denote significance using $*$ for $10 \%$, ** for $5 \%$ and $* * *$ for $1 \%$.

\begin{tabular}{lcccc}
\hline & $\begin{array}{c}(1) \\
\text { Total } \\
\text { fixed assets }\end{array}$ & $\begin{array}{c}\text { Current assets: } \\
\text { cash }\end{array}$ & $\begin{array}{c}(3) \\
\text { Current assets: } \\
\text { accounts receivable }\end{array}$ & $\begin{array}{c}(4) \\
\text { Current assets: } \\
\text { inventory }\end{array}$ \\
\hline Take-up & 793.59 & 4.86 & -1.06 & -53.85 \\
& $(116.27)$ & $(3.20)$ & $(2.65)$ & $(62.36)$ \\
& {$[0.00]^{* * *}$} & {$[0.13]$} & {$[0.69]$} & {$[0.39]$} \\
& $\{0.00\}^{* * *}$ & $\{0.24\}$ & $\{0.53\}$ & $\{0.35\}$ \\
\hline Control mean (follow-up) & 660.19 & 31.38 & 9.93 & 250.77 \\
Observations & 3,608 & 3,608 & 3,608 & 3,608 \\
\hline
\end{tabular}

Note: In this table we report the LATE estimates of the combined treatment on primary outcomes, obtained by leastsquares estimation. Below each coefficient, we report a standard error in parenthesis, a $p$-value in brackets, and a $q$-value in curly braces. Standard errors allow for clustering at the level of the individual. $q$-values are obtained using the sharpened procedure of (Benjamini et al., 2006). We denote significance using $*$ for $10 \%, * *$ for $5 \%$ and $* * *$ for $1 \%$. 


\begin{tabular}{lccccc}
\hline & $\begin{array}{c}(1) \\
\text { Household } \\
\text { income }\end{array}$ & $\begin{array}{c}(2) \\
\text { Household consumption } \\
\text { expenditure }\end{array}$ & $\begin{array}{c}(3) \\
\text { Household } \\
\text { savings }\end{array}$ & $\begin{array}{c}\text { Household } \\
\text { loans }\end{array}$ & $\begin{array}{c}\text { Household } \\
\text { assets }\end{array}$ \\
\hline Take-up & 56.96 & 23.53 & 29.76 & -41.16 & 36.92 \\
& $(22.72)$ & $(6.15)$ & $(34.71)$ & $(6.45)$ & $(25.52)$ \\
& {$[0.01]^{* *}$} & {$[0.00]^{* * *}$} & {$[0.39]$} & {$[0.00]^{* * *}$} & {$[0.15]$} \\
& $\{0.01\}^{* *}$ & $\{0.00\}^{* * *}$ & $\{0.19\}$ & $\{0.00\}^{* * *}$ & $\{0.08\}^{*}$ \\
\hline Control mean (follow-up) & 357.35 & 220.40 & 113.03 & 46.05 & 681.79 \\
Observations & 3,608 & 3,608 & 3,608 & 3,608 & 1,410 \\
\hline
\end{tabular}

Note: In this table we report the LATE estimates of the combined treatment on primary outcomes, obtained by least-squares estimation. Below each coefficient, we report a standard error in parenthesis, a $p$-value in brackets, and a $q$-value in curly braces. Standard errors allow for clustering at the level of the individual. $q$-values are obtained using the sharpened procedure of (Benjamini et al., 2006). We denote significance using $*$ for $10 \%, * *$ for $5 \%$ and $* * *$ for $1 \%$.

\begin{tabular}{lcccc}
\hline & $\begin{array}{c}(1) \\
\text { Has a } \\
\text { wage job }\end{array}$ & $\begin{array}{c}(2) \\
\text { Number of } \\
\text { wage jobs }\end{array}$ & $\begin{array}{c}(3) \\
\text { Total } \\
\text { wage hours }\end{array}$ & $\begin{array}{c}\text { Total } \\
\text { wage income }\end{array}$ \\
\hline Take-up & -0.12 & -0.12 & -6.56 & -27.68 \\
& $(0.05)$ & $(0.05)$ & $(2.36)$ & $(10.87)$ \\
& {$[0.01]^{* * *}$} & {$[0.01]^{* *}$} & {$[0.01]^{* * *}$} & {$[0.01]^{* *}$} \\
& $\{0.01\}^{* *}$ & $\{0.01\}^{* *}$ & $\{0.01\}^{* *}$ & $\{0.01\}^{* *}$ \\
\hline Control mean (follow-up) & 0.25 & 0.25 & 12.48 & 55.38 \\
Observations & 3,608 & 3,608 & 3,608 & 3,608 \\
\hline
\end{tabular}

Note: In this table we report the LATE estimates of the combined treatment on primary outcomes, obtained by least-squares estimation. Below each coefficient, we report a standard error in parenthesis, a $p$-value in brackets, and a $q$-value in curly braces. Standard errors allow for clustering at the level of the individual. $q$-values are obtained using the sharpened procedure of (Benjamini et al., 2006). We denote significance using $*$ for $10 \%, * *$ for $5 \%$ and $* * *$ for $1 \%$. 


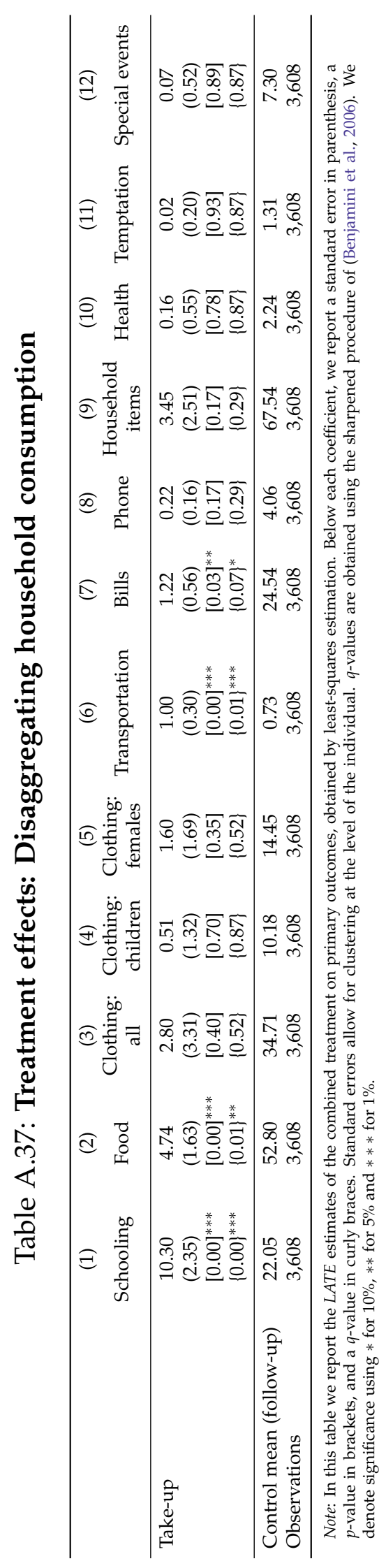




\section{Heterogeneity analysis}

In this section, we present tables illustrating heterogeneity in (i) contract take-up; (ii) usage of the flexible-repayment option; and (iii) heterogeneity in outcome variables.

\section{M.1 Heterogeneity in take-up}

Table A.38: Heterogeneity in take-up

\begin{tabular}{lccccc}
\hline & $\begin{array}{c}(1) \\
\text { Risk } \\
\text { aversion }\end{array}$ & $\begin{array}{c}\text { Loss } \\
\text { aversion }\end{array}$ & $\begin{array}{c}(3) \\
\text { Time } \\
\text { preference }\end{array}$ & $\begin{array}{c}\text { Management } \\
\text { practices }\end{array}$ & $\begin{array}{c}(5) \\
\text { Cognitive } \\
\text { ability }\end{array}$ \\
\hline Fixed * Low tercile & 0.60 & 0.54 & 0.55 & 0.47 & 0.58 \\
& $(0.051)$ & $(0.049)$ & $(0.054)$ & $(0.053)$ & $(0.051)$ \\
Fixed * Middle tercile & 0.55 & 0.57 & 0.51 & 0.63 & 0.48 \\
& $(0.057)$ & $(0.060)$ & $(0.070)$ & $(0.054)$ & $(0.063)$ \\
Fixed * High tercile & 0.43 & 0.48 & 0.52 & 0.50 & 0.51 \\
& $(0.052)$ & $(0.054)$ & $(0.045)$ & $(0.053)$ & $(0.050)$ \\
Flexible * Low tercile & 0.53 & 0.55 & 0.63 & 0.56 & 0.62 \\
& $(0.054)$ & $(0.048)$ & $(0.061)$ & $(0.055)$ & $(0.057)$ \\
Flexible * Middle tercile & 0.56 & 0.58 & 0.61 & 0.57 & 0.53 \\
& $(0.057)$ & $(0.063)$ & $(0.059)$ & $(0.054)$ & $(0.060)$ \\
Flexible * High tercile & 0.67 & 0.65 & 0.55 & 0.64 & 0.61 \\
& $(0.051)$ & $(0.054)$ & $(0.047)$ & $(0.053)$ & $(0.048)$ \\
\hline Observations & 503 & 503 & 503 & 503 & 503 \\
Test: Fixed coefficients equal & 0.058 & 0.512 & 0.858 & 0.068 & 0.406 \\
Test: Flexible coefficients equal & 0.116 & 0.395 & 0.524 & 0.491 & 0.477 \\
Test: High tercile equal & 0.001 & 0.029 & 0.623 & 0.058 & 0.161 \\
Test: Diff-in-diff (high vs low tercile) & 0.002 & 0.133 & 0.631 & 0.661 & 0.554 \\
\hline
\end{tabular}

Note: In each column of this table, the dependent variable is a dummy that is equal to 1 for individuals who took up the asset finance contract (regardless of whether it was with a fixed or flexible repayment schedule), and we investigate heterogeneous take-up by interacting each of the two treatment dummies (assignment to the fixed or flexible contract) with dummies for each of the three terciles generated when trichotomising the following (pre-specified) baseline variables: (1) risk preferences (coming from our incentivised measure, with the high tercile representing the most risk averse microenterprise owners); (2) loss aversion (again using the incentivised measure, where the high tercile represents the most loss averse microenterprise owners); (3) time preferences (with high representing the most impatient individuals, as measured using an incentivised exercise); (4) business management practices (with the high tercile representing the group with the highest score for the business management practices index); and (5) cognitive ability (with the high tercile representing those who scored highest on a series of mathematical and number recall questions). Standard errors, reported below each coefficient in parenthesis, allow for clustering at the level of the individual. Individuals from the control group (who were not given the opportunity to take up the asset finance contract) are excluded from the regressions. Below the results, we report the p-values from four tests: (i) whether the interaction terms for all the fixed contract coefficients are equal; (ii) whether the interaction terms for all the flexible contract coefficients are equal; (iii) whether the take-up rate across contracts was equal for individuals in the highest tercile of the heterogeneity measure (e.g. testing the null that, for the most risk averse or most loss averse microenterprise owners, take-up when assigned to the flexible contract was equal to take-up when assigned to the fixed contract); (iv) a difference-in-difference test: testing that the take-up differential between individuals in the lowest tercile compared to that for individuals in the highest tercile was the same across contracts (e.g. within those assigned to the fixed contract, compared the different in take-up for the most risk averse and most risk tolerant, and comparing that number for the equivalent difference for people assigned to the flexible contract). 


\section{M.2 Heterogeneity in usage of the flexible-repayment option}

Table A.39: Business shocks, repayment flexibility and risk-related heterogeneity

\begin{tabular}{|c|c|c|c|c|c|}
\hline & $\begin{array}{c}(1) \\
\text { Payment }\end{array}$ & $\begin{array}{c}(2) \\
\text { Payment }\end{array}$ & $\begin{array}{c}(3) \\
\text { Payment }\end{array}$ & $\begin{array}{c}(4) \\
\text { Payment }\end{array}$ & $\begin{array}{c}(5) \\
\text { Payment }\end{array}$ \\
\hline Positive shock & $\begin{array}{l}1.66^{* *} \\
(0.032)\end{array}$ & & $\begin{array}{c}0.52 \\
(0.464)\end{array}$ & & $\begin{array}{c}0.22 \\
(0.579)\end{array}$ \\
\hline Negative shock & $\begin{array}{l}-1.74^{*} \\
(0.053)\end{array}$ & & $\begin{array}{c}-2.28 \\
(0.350)\end{array}$ & & $\begin{array}{c}-0.59 \\
(0.748)\end{array}$ \\
\hline Medium risk & & $\begin{array}{l}-3.82^{* * *} \\
(0.002)\end{array}$ & $\begin{array}{c}-2.54 \\
(0.265)\end{array}$ & $\begin{array}{c}1.20 \\
(0.385)\end{array}$ & $\begin{array}{l}-1.30 \\
(0.452)\end{array}$ \\
\hline High risk & & $\begin{array}{l}-2.34^{* *} \\
(0.039)\end{array}$ & $\begin{array}{l}-2.35 \\
(0.343)\end{array}$ & $\begin{array}{c}0.21 \\
(0.877)\end{array}$ & $\begin{array}{l}-1.30 \\
(0.520)\end{array}$ \\
\hline Positive ${ }^{*}$ Medium risk & & & $\begin{array}{c}-0.34 \\
(0.666)\end{array}$ & & $\begin{array}{c}0.63 \\
(0.350)\end{array}$ \\
\hline Positive ${ }^{*}$ High risk & & & $\begin{array}{l}2.45^{* * *} \\
(0.001)\end{array}$ & & $\begin{array}{l}2.47^{* * *} \\
(0.000)\end{array}$ \\
\hline Negative ${ }^{*}$ Medium risk & & & $\begin{array}{c}1.33 \\
(0.640)\end{array}$ & & $\begin{array}{l}-2.64 \\
(0.209)\end{array}$ \\
\hline Negative ${ }^{*}$ High risk & & & $\begin{array}{c}0.45 \\
(0.862)\end{array}$ & & $\begin{array}{l}-0.67 \\
(0.777)\end{array}$ \\
\hline Observations & 480 & 492 & 480 & 492 & 480 \\
\hline Individuals & 123 & 123 & 123 & 123 & 123 \\
\hline $\begin{array}{l}\text { Dependent variable mean } \\
\text { Heterogeneity measure }\end{array}$ & 20.02 & \multicolumn{2}{|c|}{ Risk exposure } & \multicolumn{2}{|c|}{ Risk aversion } \\
\hline
\end{tabular}

Note: In this table we investigate how microenterprises respond to business shocks in terms of the payments they made under the flexible-repayment contract. Shocks are defined as the percentage change in monthly business profits, compared to the value six months prior. In each column, the dependent variable is the cumulative payment made in the last six months for individuals under the flexible-repayment contract, which allowed them to pay more than their obligation of $2.5 \%$ of the asset value each month. Columns 2 and 3 include a measure of baseline microenterprise 'risk exposure', constructed as the standard deviation of the previous three months of business profits at baseline. Columns 4 and 5 use the incentivised measure of baseline risk aversion for microenterprise owners. Both risk variables are constructed using a tercile split, with 'high risk' referring to the most risk exposed microenterprises / the most risk-averse microentrepreneurs respectively, 'medium risk' referring to the middle tercile of risk exposure / risk aversion, and the omitted category being the least risk exposed / least risk-averse. Standard errors, reported below each coefficient in parenthesis, allow for clustering at the level of the individual. We denote significance using $*$ for $10 \%, * *$ for $5 \%$ and $* * *$ for $1 \%$. 


\section{M.3 Heterogeneity in outcomes}

\section{Table A.40: Outcome heterogeneity by baseline loss tercile}

\begin{tabular}{|c|c|c|c|c|c|c|c|}
\hline & $\begin{array}{c}\text { (1) } \\
\text { Number of } \\
\text { businesses }\end{array}$ & $\begin{array}{c}\text { (2) } \\
\text { Business } \\
\text { profits }\end{array}$ & $\begin{array}{c}\text { (3) } \\
\text { Business } \\
\text { assets }\end{array}$ & $\begin{array}{c}(4) \\
\text { Fixed } \\
\text { assets }\end{array}$ & $\begin{array}{l}\text { (5) } \\
\text { Household } \\
\text { income }\end{array}$ & $\begin{array}{c}(6) \\
\text { Household } \\
\text { consumption }\end{array}$ & $\begin{array}{c}(7) \\
\text { Householc } \\
\text { savings }\end{array}$ \\
\hline Medium loss aversion & $\begin{array}{c}0.07 \\
(0.058)\end{array}$ & $\begin{array}{c}6.81 \\
(22.970)\end{array}$ & $\begin{array}{c}150.98 \\
(196.211)\end{array}$ & $\begin{array}{c}-21.72 \\
(144.766)\end{array}$ & $\begin{array}{c}33.26 \\
(30.609)\end{array}$ & $\begin{array}{c}-3.60 \\
(7.438)\end{array}$ & $\begin{array}{c}-43.20 \\
(40.994)\end{array}$ \\
\hline High loss aversion & $\begin{array}{c}0.05 \\
(0.050)\end{array}$ & $\begin{array}{c}-0.20 \\
(19.800)\end{array}$ & $\begin{array}{c}66.35 \\
(173.000)\end{array}$ & $\begin{array}{c}15.32 \\
(127.059)\end{array}$ & $\begin{array}{c}0.36 \\
(24.149)\end{array}$ & $\begin{array}{c}-6.15 \\
(6.881)\end{array}$ & $\begin{array}{c}29.64 \\
(35.118)\end{array}$ \\
\hline Fixed ${ }^{*}$ Low loss aversion & $\begin{array}{l}0.19^{* * *} \\
(0.044)\end{array}$ & $\begin{array}{l}52.60^{* * *} \\
(17.905)\end{array}$ & $\begin{array}{l}613.45^{* * *} \\
(178.935)\end{array}$ & $\begin{array}{l}549.81^{* * *} \\
(145.343)\end{array}$ & $\begin{array}{c}48.64^{* *} \\
(22.792)\end{array}$ & $\begin{array}{l}12.71^{* *} \\
(5.941)\end{array}$ & $\begin{array}{c}1.34 \\
(42.268)\end{array}$ \\
\hline Fixed ${ }^{*}$ Medium loss aversion & $\begin{array}{c}0.02 \\
(0.054)\end{array}$ & $\begin{array}{c}23.01 \\
(22.469)\end{array}$ & $\begin{array}{c}359.12 \\
(218.670)\end{array}$ & $\begin{array}{l}507.09^{* * *} \\
(165.959)\end{array}$ & $\begin{array}{c}16.50 \\
(31.760)\end{array}$ & $\begin{array}{l}16.65^{* *} \\
(8.181)\end{array}$ & $\begin{array}{c}55.49 \\
(45.020)\end{array}$ \\
\hline Fixed * High loss aversion & $\begin{array}{c}0.03 \\
(0.051)\end{array}$ & $\begin{array}{c}4.79 \\
(20.139)\end{array}$ & $\begin{array}{c}248.79 \\
(162.499)\end{array}$ & $\begin{array}{l}389.17^{* * *} \\
(125.779)\end{array}$ & $\begin{array}{c}6.95 \\
(26.024)\end{array}$ & $\begin{array}{l}12.18^{*} \\
(7.091)\end{array}$ & $\begin{array}{c}-45.87 \\
(31.765)\end{array}$ \\
\hline Flexible * Low loss aversion & $\begin{array}{l}0.11^{* *} \\
(0.048)\end{array}$ & $\begin{array}{c}36.99^{* *} \\
(18.087)\end{array}$ & $\begin{array}{l}557.64^{* * *} \\
(157.560)\end{array}$ & $\begin{array}{l}552.46^{* * *} \\
(123.042)\end{array}$ & $\begin{array}{c}42.72^{* *} \\
(21.651)\end{array}$ & $\begin{array}{c}8.08 \\
(6.341)\end{array}$ & $\begin{array}{c}38.33 \\
(36.572)\end{array}$ \\
\hline Flexible * Medium loss aversion & $\begin{array}{c}0.08 \\
(0.055)\end{array}$ & $\begin{array}{c}14.14 \\
(22.370)\end{array}$ & $\begin{array}{c}48.44 \\
(198.810)\end{array}$ & $\begin{array}{c}222.47 \\
(166.009)\end{array}$ & $\begin{array}{c}6.06 \\
(31.732)\end{array}$ & $\begin{array}{l}15.04^{*} \\
(8.492)\end{array}$ & $\begin{array}{c}90.66^{*} \\
(46.435)\end{array}$ \\
\hline Flexible ${ }^{*}$ High loss aversion & $\begin{array}{c}0.09^{*} \\
(0.049)\end{array}$ & $\begin{array}{c}18.66 \\
(19.648) \\
\end{array}$ & $\begin{array}{c}371.08^{*} \\
(194.265)\end{array}$ & $\begin{array}{c}321.71^{* *} \\
(142.489)\end{array}$ & $\begin{array}{c}48.80^{*} \\
(26.306)\end{array}$ & $\begin{array}{l}18.24^{* *} \\
(7.592)\end{array}$ & $\begin{array}{c}-13.38 \\
(36.399)\end{array}$ \\
\hline Observations & 3608 & 3608 & 3608 & 3608 & 3608 & 3608 & 3608 \\
\hline Test: Fixed coefficients equal & 0.019 & 0.211 & 0.338 & 0.688 & 0.466 & 0.904 & 0.175 \\
\hline Test: Flexible coefficients equal & 0.931 & 0.693 & 0.151 & 0.258 & 0.546 & 0.593 & 0.184 \\
\hline Test: Diff-in-diff (high vs low loss aversion) & 0.031 & 0.268 & 0.498 & 0.743 & 0.179 & 0.302 & 0.939 \\
\hline
\end{tabular}

Note: In this table we investigate heterogeneous treatment effects by interacting each of the two treatment dummies (assignment to the fixed or flexible contract) with dummies for each of the three baseline loss terciles (where low, medium and high loss aversion refers to individuals who were in the bottom, middle and highest terciles of measured loss aversion using the baseline loss aversion elicitation task). The omitted category represents individuals in the control group from the lowest tercile of loss aversion. Standard errors, reported below each coefficient in parenthesis, allow for clustering at the level of the individual. We denote significance using $*$ for $10 \%$, $* *$ for $5 \%$ and $* * *$ for $1 \%$. In the bottom three rows of the table, we report p-values for two null hypotheses: (i) the null hypothesis that the effect of assignment to the fixed contract is equal across three terciles of loss aversion; (ii) the null hypothesis that the effect of assignment to the flexible contract is equal across loss terciles; and (iii) a difference-in-difference test: testing the null that the difference in outcomes between the least loss averse and the most loss averse was the same across the two assignment groups. 
Table A.41: Outcome heterogeneity by baseline time-preference tercile

\begin{tabular}{lccccccc}
\hline & $\begin{array}{c}(1) \\
\text { Number of } \\
\text { businesses }\end{array}$ & $\begin{array}{c}(2) \\
\text { Business } \\
\text { profits }\end{array}$ & $\begin{array}{c}(3) \\
\text { Business } \\
\text { assets }\end{array}$ & $\begin{array}{c}(4) \\
\text { Fixed } \\
\text { assets }\end{array}$ & $\begin{array}{c}\text { Household } \\
\text { income }\end{array}$ & $\begin{array}{c}(5) \\
\text { Household } \\
\text { consumption }\end{array}$ & $\begin{array}{c}(7) \\
\text { Household } \\
\text { savings }\end{array}$ \\
\hline Medium impatience & -0.09 & -28.00 & $-361.89^{*}$ & $-288.46^{*}$ & -10.34 & 6.09 & -46.28 \\
& $(0.061)$ & $(24.338)$ & $(213.110)$ & $(165.977)$ & $(31.784)$ & $(8.258)$ & $(45.098)$ \\
High impatience & -0.03 & -14.74 & $-379.03^{*}$ & $-357.67^{* *}$ & -1.77 & -6.01 & -29.37 \\
& $(0.050)$ & $(20.472)$ & $(198.919)$ & $(144.543)$ & $(24.713)$ & $(6.650)$ & $(37.534)$ \\
Fixed * Low impatience & 0.08 & 14.12 & 26.83 & 257.53 & 37.07 & $16.95^{* *}$ & -26.41 \\
& $(0.049)$ & $(20.907)$ & $(211.702)$ & $(163.505)$ & $(26.885)$ & $(7.736)$ & $(48.278)$ \\
Fixed * Medium impatience & $0.25^{* * *}$ & $68.29^{* * *}$ & $831.31^{* * *}$ & $775.35^{* * *}$ & 40.46 & 3.51 & -3.47 \\
& $(0.058)$ & $(23.505)$ & $(228.292)$ & $(201.884)$ & $(33.098)$ & $(8.791)$ & $(45.534)$ \\
Fixed * High impatience & 0.04 & 19.73 & $525.18^{* * *}$ & $513.37^{* * *}$ & 13.16 & $16.23^{* * *}$ & 16.36 \\
& $(0.042)$ & $(16.423)$ & $(148.809)$ & $(110.339)$ & $(20.503)$ & $(5.504)$ & $(31.159)$ \\
Flexible * Low impatience & 0.08 & 32.06 & $377.62^{*}$ & $348.87^{* *}$ & $58.03^{* *}$ & 10.67 & -29.93 \\
& $(0.050)$ & $(21.187)$ & $(226.949)$ & $(160.718)$ & $(28.493)$ & $(8.190)$ & $(40.791)$ \\
Flexible * Medium impatience & $0.18^{* * *}$ & $45.28^{* *}$ & $489.00^{* * *}$ & $426.27^{* * *}$ & $54.29^{*}$ & 5.69 & 47.47 \\
& $(0.060)$ & $(22.549)$ & $(184.864)$ & $(154.830)$ & $(30.359)$ & $(8.225)$ & $(44.215)$ \\
Flexible * High impatience & 0.06 & 11.43 & $341.14^{* *}$ & $438.06^{* * *}$ & 12.47 & $18.38^{* * *}$ & $67.00^{*}$ \\
& $(0.045)$ & $(17.540)$ & $(157.870)$ & $(116.777)$ & $(22.410)$ & $(6.175)$ & $(39.267)$ \\
\hline Observations & 3608 & 3608 & 3608 & 3608 & 3608 & 3608 & 3608 \\
Test: Fixed coefficients equal & 0.016 & 0.184 & 0.034 & 0.147 & 0.695 & 0.436 & 0.752 \\
Test: Flexible coefficients equal & 0.249 & 0.486 & 0.834 & 0.907 & 0.384 & 0.464 & 0.214 \\
Test: Diff-in-diff (high vs low impatience) & 0.847 & 0.281 & 0.037 & 0.388 & 0.541 & 0.437 & 0.351 \\
\hline
\end{tabular}

Note: In this table we investigate heterogeneous treatment effects by interacting each of the two treatment dummies (assignment to the fixed or flexible contract) with dummies for each of the three baseline time preference terciles (where low, medium and high time preferences refers to individuals who were in the bottom, middle and highest terciles of measured impatience using the baseline time preference elicitation task). The omitted category represents individuals in the control group from the lowest tercile of time preferences (most patient). Standard errors, reported below each coefficient in parenthesis, allow for clustering at the level of the individual. We denote significance using $*$ for $10 \%$, $* *$ for $5 \%$ and $* * *$ for $1 \%$. In the bottom three rows of the table, we report p-values for two null hypotheses: (i) the null hypothesis that the effect of assignment to the fixed contract is equal across three terciles of impatience; (ii) the null hypothesis that the effect of assignment to the flexible contract is equal across time preference terciles; and (iii) a difference-in-difference test: testing the null that the difference in outcomes between the least patient and the most patient was the same across the two assignment groups. 


\section{Table A.42: Outcome heterogeneity by baseline management tercile}

\begin{tabular}{|c|c|c|c|c|c|c|c|}
\hline & $\begin{array}{c}\text { (1) } \\
\text { Number of } \\
\text { businesses }\end{array}$ & $\begin{array}{l}\text { (2) } \\
\text { Business } \\
\text { profits }\end{array}$ & $\begin{array}{l}\text { (3) } \\
\text { Business } \\
\text { assets }\end{array}$ & $\begin{array}{c}(4) \\
\text { Fixed } \\
\text { assets }\end{array}$ & $\begin{array}{l}\text { (5) } \\
\text { Household } \\
\text { income }\end{array}$ & $\begin{array}{c}(6) \\
\text { Household } \\
\text { consumption }\end{array}$ & $\begin{array}{l}\text { (7) } \\
\text { Household } \\
\text { savings }\end{array}$ \\
\hline Medium management & $\begin{array}{c}-0.06 \\
(0.053)\end{array}$ & $\begin{array}{c}3.43 \\
(20.484)\end{array}$ & $\begin{array}{c}72.58 \\
(184.064)\end{array}$ & $\begin{array}{c}-6.77 \\
(138.383)\end{array}$ & $\begin{array}{c}7.44 \\
(25.502)\end{array}$ & $\begin{array}{c}2.19 \\
(7.266)\end{array}$ & $\begin{array}{c}50.77 \\
(34.008)\end{array}$ \\
\hline High management & $\begin{array}{c}-0.06 \\
(0.054)\end{array}$ & $\begin{array}{c}16.03 \\
(22.213)\end{array}$ & $\begin{array}{c}-67.05 \\
(206.524)\end{array}$ & $\begin{array}{c}-35.51 \\
(143.519)\end{array}$ & $\begin{array}{c}40.54 \\
(28.411)\end{array}$ & $\begin{array}{c}5.02 \\
(7.982)\end{array}$ & $\begin{array}{c}8.28 \\
(42.609)\end{array}$ \\
\hline Fixed * Low management & $\begin{array}{c}0.09^{* *} \\
(0.046)\end{array}$ & $\begin{array}{c}33.39^{*} \\
(18.097)\end{array}$ & $\begin{array}{l}573.34^{* * *} \\
(183.984)\end{array}$ & $\begin{array}{l}552.38^{* * * *} \\
(153.301)\end{array}$ & $\begin{array}{c}21.77 \\
(24.395)\end{array}$ & $\begin{array}{c}19.83^{* * *} \\
(6.931)\end{array}$ & $\begin{array}{c}3.32 \\
(32.687)\end{array}$ \\
\hline Fixed * Medium management & $\begin{array}{l}0.13^{* *} \\
(0.054)\end{array}$ & $\begin{array}{l}42.98^{* *} \\
(20.885)\end{array}$ & $\begin{array}{l}483.15^{* * *} \\
(176.776)\end{array}$ & $\begin{array}{l}570.50^{* * * *} \\
(133.062)\end{array}$ & $\begin{array}{c}43.28^{*} \\
(25.268)\end{array}$ & $\begin{array}{l}11.95^{*} \\
(6.789)\end{array}$ & $\begin{array}{c}-52.58 \\
(32.936)\end{array}$ \\
\hline Fixed * High management & $\begin{array}{c}0.08 \\
(0.050)\end{array}$ & $\begin{array}{c}11.08 \\
(20.776)\end{array}$ & $\begin{array}{c}267.14 \\
(189.332)\end{array}$ & $\begin{array}{c}340.75^{* *} \\
(142.258)\end{array}$ & $\begin{array}{c}15.97 \\
(27.503)\end{array}$ & $\begin{array}{c}7.87 \\
(7.307)\end{array}$ & $\begin{array}{c}50.71 \\
(49.504)\end{array}$ \\
\hline Flexible * Low management & $\begin{array}{c}-0.03 \\
(0.053)\end{array}$ & $\begin{array}{c}5.24 \\
(19.443)\end{array}$ & $\begin{array}{c}418.50^{* *} \\
(188.853)\end{array}$ & $\begin{array}{l}311.22^{* *} \\
(140.923)\end{array}$ & $\begin{array}{c}22.95 \\
(24.486)\end{array}$ & $\begin{array}{c}7.43 \\
(7.259)\end{array}$ & $\begin{array}{c}8.85 \\
(31.080)\end{array}$ \\
\hline Flexible * Medium management & $\begin{array}{l}0.17^{* * * *} \\
(0.052)\end{array}$ & $\begin{array}{l}54.79^{* * *} \\
(20.121)\end{array}$ & $\begin{array}{c}319.16^{*} \\
(163.441)\end{array}$ & $\begin{array}{l}396.41^{* * * *} \\
(136.239)\end{array}$ & $\begin{array}{l}77.99^{* * * *} \\
(25.342)\end{array}$ & $\begin{array}{l}15.64^{* *} \\
(6.702)\end{array}$ & $\begin{array}{c}48.05 \\
(37.931)\end{array}$ \\
\hline Flexible * High management & $\begin{array}{l}0.14^{* * *} \\
(0.047)\end{array}$ & $\begin{array}{c}16.36 \\
(19.506)\end{array}$ & $\begin{array}{l}397.49 * * \\
(179.387)\end{array}$ & $\begin{array}{l}479.40^{* * *} \\
(130.719)\end{array}$ & $\begin{array}{c}6.13 \\
(26.794)\end{array}$ & $\begin{array}{l}15.30^{*} \\
(8.173)\end{array}$ & $\begin{array}{c}51.43 \\
(50.891)\end{array}$ \\
\hline Observations & 3608 & 3608 & 3608 & 3608 & 3608 & 3608 & 3608 \\
\hline Test: Fixed coefficients equal & 0.811 & 0.546 & 0.505 & 0.450 & 0.738 & 0.480 & 0.208 \\
\hline Test: Flexible coefficients equal & 0.013 & 0.190 & 0.912 & 0.685 & 0.124 & 0.666 & 0.638 \\
\hline Test: Diff-in-diff (high vs low management) & 0.005 & 0.182 & 0.248 & 0.072 & 0.754 & 0.054 & 0.934 \\
\hline
\end{tabular}

Note: In this table we investigate heterogeneous treatment effects by interacting each of the two treatment dummies (assignment to the fixed or flexible contract) with dummies for each of the three management practices terciles (where low, medium and high refers to individuals who were in the bottom, middle and highest terciles of baseline management practices). The omitted category represents individuals in the control group from the lowest tercile of management practices. Standard errors, reported below each coefficient in parenthesis, allow for clustering at the level of the individual. We denote significance using $*$ for $10 \%, * *$ for $5 \%$ and $* * *$ for $1 \%$. In the bottom three rows of the table, we report p-values for two null hypotheses: (i) the null hypothesis that the effect of assignment to the fixed contract is equal across three terciles of management practices; (ii) the null hypothesis that the effect of assignment to the flexible contract is equal across management terciles; and (iii) a difference-in-difference test: testing the null that the difference in outcomes between the lowest management practices and the highest management practices was the same across the two assignment groups. 
Table A.43: Outcome heterogeneity by baseline numeracy tercile

\begin{tabular}{|c|c|c|c|c|c|c|c|}
\hline & $\begin{array}{c}\text { (1) } \\
\text { Number of } \\
\text { businesses }\end{array}$ & $\begin{array}{l}\text { (2) } \\
\text { Business } \\
\text { profits }\end{array}$ & $\begin{array}{c}\text { (3) } \\
\text { Business } \\
\text { assets }\end{array}$ & $\begin{array}{c}(4) \\
\text { Fixed } \\
\text { assets }\end{array}$ & $\begin{array}{l}\quad(5) \\
\text { Household } \\
\text { income }\end{array}$ & $\begin{array}{c}(6) \\
\text { Household } \\
\text { consumption }\end{array}$ & $\begin{array}{l}\text { (7) } \\
\text { Household } \\
\text { savings }\end{array}$ \\
\hline Medium numeracy & $\begin{array}{l}-0.10^{*} \\
(0.056)\end{array}$ & $\begin{array}{c}-28.63 \\
(22.861)\end{array}$ & $\begin{array}{c}277.83 \\
(200.793)\end{array}$ & $\begin{array}{c}223.28 \\
(144.585)\end{array}$ & $\begin{array}{c}-27.98 \\
(29.154)\end{array}$ & $\begin{array}{c}1.16 \\
(7.938)\end{array}$ & $\begin{array}{c}19.59 \\
(45.849)\end{array}$ \\
\hline High numeracy & $\begin{array}{c}-0.04 \\
(0.051)\end{array}$ & $\begin{array}{c}-8.01 \\
(21.190)\end{array}$ & $\begin{array}{l}411.87^{* *} \\
(182.284)\end{array}$ & $\begin{array}{c}266.09^{* * *} \\
(131.743)\end{array}$ & $\begin{array}{c}-10.05 \\
(27.460)\end{array}$ & $\begin{array}{c}7.89 \\
(6.931)\end{array}$ & $\begin{array}{c}-17.82 \\
(37.324)\end{array}$ \\
\hline Fixed ${ }^{*}$ Low numeracy & $\begin{array}{c}0.04 \\
(0.043)\end{array}$ & $\begin{array}{c}15.79 \\
(18.893)\end{array}$ & $\begin{array}{l}796.03^{* * * *} \\
(194.907)\end{array}$ & $\begin{array}{l}716.22^{* * * *} \\
(156.982)\end{array}$ & $\begin{array}{c}18.95 \\
(25.449)\end{array}$ & $\begin{array}{c}21.86^{* * * *} \\
(6.723)\end{array}$ & $\begin{array}{c}-37.55 \\
(35.524)\end{array}$ \\
\hline Fixed * Medium numeracy & $\begin{array}{l}0.18^{* * *} \\
(0.057)\end{array}$ & $\begin{array}{l}47.81^{* *} \\
(22.312)\end{array}$ & $\begin{array}{c}352.97^{*} \\
(213.937)\end{array}$ & $\begin{array}{l}465.98^{* * *} \\
(159.591)\end{array}$ & $\begin{array}{c}21.68 \\
(27.207)\end{array}$ & $\begin{array}{c}5.88 \\
(7.637)\end{array}$ & $\begin{array}{c}-13.38 \\
(47.127)\end{array}$ \\
\hline Fixed * High numeracy & $\begin{array}{c}0.09^{*} \\
(0.049)\end{array}$ & $\begin{array}{c}26.02 \\
(18.724)\end{array}$ & $\begin{array}{c}180.18 \\
(159.540)\end{array}$ & $\begin{array}{l}301.63^{* *} \\
(125.574)\end{array}$ & $\begin{array}{c}37.31 \\
(23.529)\end{array}$ & $\begin{array}{c}10.65 \\
(6.545)\end{array}$ & $\begin{array}{c}40.76 \\
(39.953)\end{array}$ \\
\hline Flexible * Low numeracy & $\begin{array}{c}0.03 \\
(0.049)\end{array}$ & $\begin{array}{c}16.60 \\
(19.926)\end{array}$ & $\begin{array}{l}718.32^{* * *} \\
(187.015)\end{array}$ & $\begin{array}{l}572.15^{* * *} \\
(155.273)\end{array}$ & $\begin{array}{c}19.04 \\
(27.064)\end{array}$ & $\begin{array}{l}18.36^{* *} \\
(7.634)\end{array}$ & $\begin{array}{c}22.09 \\
(40.301)\end{array}$ \\
\hline Flexible * Medium numeracy & $\begin{array}{c}0.15^{* *} \\
(0.059)\end{array}$ & $\begin{array}{c}39.38^{*} \\
(23.082)\end{array}$ & $\begin{array}{c}281.43 \\
(195.176)\end{array}$ & $\begin{array}{c}196.57 \\
(139.984)\end{array}$ & $\begin{array}{c}47.33 \\
(29.406)\end{array}$ & $\begin{array}{c}13.16 \\
(8.197)\end{array}$ & $\begin{array}{c}-13.06 \\
(41.825)\end{array}$ \\
\hline Flexible * High numeracy & $\begin{array}{l}0.11^{* *} \\
(0.049)\end{array}$ & $\begin{array}{c}23.13 \\
(18.350)\end{array}$ & $\begin{array}{c}170.60 \\
(149.916)\end{array}$ & $\begin{array}{l}383.70^{* * *} \\
(114.096)\end{array}$ & $\begin{array}{c}41.48^{*} \\
(23.343)\end{array}$ & $\begin{array}{c}8.60 \\
(6.328)\end{array}$ & $\begin{array}{c}73.58^{*} \\
(40.975)\end{array}$ \\
\hline Observations & 3608 & 3608 & 3608 & 3608 & 3608 & 3608 & 3608 \\
\hline Test: Fixed coefficients equal & 0.173 & 0.548 & 0.050 & 0.126 & 0.849 & 0.264 & 0.330 \\
\hline Test: Flexible coefficients equal & 0.276 & 0.758 & 0.072 & 0.191 & 0.754 & 0.617 & 0.347 \\
\hline Test: Diff-in-diff (high vs low numeracy) & 0.562 & 0.882 & 0.777 & 0.279 & 0.904 & 0.884 & 0.668 \\
\hline
\end{tabular}

Note: In this table we investigate heterogeneous treatment effects by interacting each of the two treatment dummies (assignment to the fixed or flexible contract) with dummies for each of the three baseline math score terciles (where low, medium and high refers to individuals who were in the bottom, middle and highest terciles of measured maths score using the baseline task). The omitted category represents individuals in the control group from the lowest tercile of maths score. Standard errors, reported below each coefficient in parenthesis, allow for clustering at the level of the individual. We denote significance using $*$ for $10 \%, * *$ for $5 \%$ and $* * *$ for $1 \%$. In the bottom three rows of the table, we report p-values for two null hypotheses: (i) the null hypothesis that the effect of assignment to the fixed contract is equal across three terciles of maths score; (ii) the null hypothesis that the effect of assignment to the flexible contract is equal across maths score terciles; and (iii) a difference-in-difference test: testing the null that the difference in outcomes between the lowest maths score and the highest maths score was the same across the two assignment groups. 
Table A.44: Outcome heterogeneity by baseline risk tercile: LATE

\begin{tabular}{|c|c|c|c|c|c|c|c|}
\hline & $\begin{array}{c}\text { (1) } \\
\text { Number of } \\
\text { businesses }\end{array}$ & $\begin{array}{l}\text { (2) } \\
\text { Business } \\
\text { profits }\end{array}$ & $\begin{array}{l}\text { (3) } \\
\text { Business } \\
\text { assets }\end{array}$ & $\begin{array}{l}(4) \\
\text { Fixed } \\
\text { assets }\end{array}$ & $\begin{array}{c}(5) \\
\text { Household } \\
\text { income }\end{array}$ & $\begin{array}{c}(6) \\
\text { Household } \\
\text { consumption }\end{array}$ & $\begin{array}{c}(7) \\
\text { Household } \\
\text { savings }\end{array}$ \\
\hline Medium & $\begin{array}{l}0.14^{* *} \\
(0.057)\end{array}$ & $\begin{array}{c}25.00 \\
(20.618)\end{array}$ & $\begin{array}{c}35.08 \\
(178.371)\end{array}$ & $\begin{array}{c}-43.84 \\
(134.269)\end{array}$ & $\begin{array}{c}31.66 \\
(26.935)\end{array}$ & $\begin{array}{c}4.18 \\
(8.533)\end{array}$ & $\begin{array}{c}20.11 \\
(38.435)\end{array}$ \\
\hline High & $\begin{array}{c}0.14^{* *} \\
(0.057)\end{array}$ & $\begin{array}{c}36.34^{*} \\
(21.395)\end{array}$ & $\begin{array}{c}441.08^{* *} \\
(182.882)\end{array}$ & $\begin{array}{c}152.91 \\
(130.043)\end{array}$ & $\begin{array}{c}12.93 \\
(27.529)\end{array}$ & $\begin{array}{c}7.19 \\
(8.978)\end{array}$ & $\begin{array}{c}-30.59 \\
(47.417)\end{array}$ \\
\hline Fixed $*$ Low & $\begin{array}{l}0.49^{* * *} \\
(0.089)\end{array}$ & $\begin{array}{c}123.26^{* * *} \\
(33.073)\end{array}$ & $\begin{array}{c}1298.98^{* * *} \\
(315.768)\end{array}$ & $\begin{array}{c}1120.52^{* * *} \\
(247.783)\end{array}$ & $\begin{array}{l}107.42^{* *} \\
(42.467)\end{array}$ & $\begin{array}{l}59.67^{* * *} \\
(14.640)\end{array}$ & $\begin{array}{c}10.24 \\
(78.113)\end{array}$ \\
\hline Fixed * Medium & $\begin{array}{c}0.02 \\
(0.091)\end{array}$ & $\begin{array}{c}33.10 \\
(34.193)\end{array}$ & $\begin{array}{l}848.33^{* *} \\
(333.646)\end{array}$ & $\begin{array}{l}975.89^{* * *} \\
(265.182)\end{array}$ & $\begin{array}{c}10.97 \\
(45.956)\end{array}$ & $\begin{array}{l}30.43^{* * *} \\
(11.311)\end{array}$ & $\begin{array}{c}-17.22 \\
(67.868)\end{array}$ \\
\hline Fixed ${ }^{*}$ High & $\begin{array}{c}0.04 \\
(0.118)\end{array}$ & $\begin{array}{c}-6.22 \\
(48.048)\end{array}$ & $\begin{array}{c}-70.85 \\
(383.753)\end{array}$ & $\begin{array}{c}483.82^{*} \\
(246.461)\end{array}$ & $\begin{array}{c}33.35 \\
(60.181)\end{array}$ & $\begin{array}{c}-8.62 \\
(17.935)\end{array}$ & $\begin{array}{c}-11.49 \\
(82.622)\end{array}$ \\
\hline Flexible * Low & $\begin{array}{l}0.36^{* * *} \\
(0.122)\end{array}$ & $\begin{array}{c}83.31^{*} \\
(44.451)\end{array}$ & $\begin{array}{c}617.01^{*} \\
(347.104)\end{array}$ & $\begin{array}{c}531.78^{*} \\
(274.036)\end{array}$ & $\begin{array}{c}81.52 \\
(57.375)\end{array}$ & $\begin{array}{c}6.42 \\
(17.469)\end{array}$ & $\begin{array}{c}-31.41 \\
(78.727)\end{array}$ \\
\hline Flexible * Medium & $\begin{array}{c}0.09 \\
(0.093)\end{array}$ & $\begin{array}{c}33.91 \\
(37.185)\end{array}$ & $\begin{array}{c}1032.67^{* * *} \\
(380.007)\end{array}$ & $\begin{array}{l}758.97^{* * *} \\
(278.083)\end{array}$ & $\begin{array}{c}44.09 \\
(49.315)\end{array}$ & $\begin{array}{l}29.63^{* *} \\
(14.133)\end{array}$ & $\begin{array}{c}56.80 \\
(96.955)\end{array}$ \\
\hline Flexible * High & $\begin{array}{c}0.13^{*} \\
(0.073)\end{array}$ & $\begin{array}{c}29.31 \\
(29.630)\end{array}$ & $\begin{array}{c}212.57 \\
(250.236)\end{array}$ & $\begin{array}{l}603.85^{* * *} \\
(179.671)\end{array}$ & $\begin{array}{c}75.90^{*} \\
(39.682)\end{array}$ & $\begin{array}{l}40.76^{* * *} \\
(13.812)\end{array}$ & $\begin{array}{l}139.40^{*} \\
(71.049)\end{array}$ \\
\hline Observations & 3608 & 3608 & 3608 & 3608 & 3608 & 3608 & 3608 \\
\hline Test: Fixed coefficients equal & 0.000 & 0.041 & 0.023 & 0.165 & 0.287 & 0.012 & 0.966 \\
\hline Test: Flexible coefficients equal & 0.164 & 0.569 & 0.208 & 0.842 & 0.850 & 0.281 & 0.327 \\
\hline Test: Diff-in-diff (high vs low tericle) & 0.171 & 0.214 & 0.062 & 0.077 & 0.405 & 0.000 & 0.137 \\
\hline
\end{tabular}

Note: In this table we investigate heterogeneous treatment effects using local average treatment effect estimates, where we instrument take-up with assignment to either of the two treatment contracts (fixed- or flexible-repayment), and interact with dummies for each of the three baseline heterogeneity terciles (where 'Low', 'Medium' and 'High' refers to individuals who were in the bottom, middle and highest terciles of the particular heterogeneity variable, as done in the equivalent table for the ITT results). The omitted category represents individuals in the control group from the lowest tercile. Standard errors, reported below each coefficient in parenthesis, allow for clustering at the level of the individual. We denote significance using $*$ for $10 \%, * *$ for $5 \%$ and $* * *$ for $1 \%$. In the bottom three rows of the table, we report p-values for three null hypotheses: (i) the null hypothesis that the effect of take-up of the fixed contract is equal across three terciles; (ii) the null hypothesis that the effect of take-up of the flexible contract is equal across terciles; and (iii) a difference-in-difference test: testing the null that the difference in outcomes between the lowest and highest tercile was the same across the two assignment groups. 
Table A.45: Outcome heterogeneity by baseline loss tercile: LATE

\begin{tabular}{|c|c|c|c|c|c|c|c|}
\hline & $\begin{array}{c}\text { (1) } \\
\text { Number of } \\
\text { businesses }\end{array}$ & $\begin{array}{l}\text { (2) } \\
\text { Business } \\
\text { profits }\end{array}$ & $\begin{array}{l}\text { (3) } \\
\text { Business } \\
\text { assets }\end{array}$ & $\begin{array}{c}(4) \\
\text { Fixed } \\
\text { assets }\end{array}$ & $\begin{array}{l}\text { (5) } \\
\text { Household } \\
\text { income }\end{array}$ & $\begin{array}{c}\text { (6) } \\
\text { Household } \\
\text { consumption }\end{array}$ & $\begin{array}{c}\text { (7) } \\
\text { Household } \\
\text { savings }\end{array}$ \\
\hline Medium & $\begin{array}{c}0.10 \\
(0.062)\end{array}$ & $\begin{array}{c}11.87 \\
(23.940)\end{array}$ & $\begin{array}{c}147.57 \\
(197.581)\end{array}$ & $\begin{array}{c}-18.68 \\
(144.721)\end{array}$ & $\begin{array}{c}35.81 \\
(31.731)\end{array}$ & $\begin{array}{c}2.48 \\
(8.400)\end{array}$ & $\begin{array}{c}-51.49 \\
(41.404)\end{array}$ \\
\hline High & $\begin{array}{c}0.08 \\
(0.051)\end{array}$ & $\begin{array}{c}5.72 \\
(19.511)\end{array}$ & $\begin{array}{c}80.58 \\
(168.084)\end{array}$ & $\begin{array}{c}44.01 \\
(122.010)\end{array}$ & $\begin{array}{c}4.08 \\
(23.499)\end{array}$ & $\begin{array}{c}1.55 \\
(7.528)\end{array}$ & $\begin{array}{c}21.01 \\
(33.923)\end{array}$ \\
\hline Fixed * Low & $\begin{array}{l}0.44^{* * *} \\
(0.088)\end{array}$ & $\begin{array}{l}111.63^{* * *} \\
(33.657)\end{array}$ & $\begin{array}{c}1130.52^{* * *} \\
(316.958)\end{array}$ & $\begin{array}{c}1037.13^{* * *} \\
(250.797)\end{array}$ & $\begin{array}{l}97.50^{* *} \\
(41.368)\end{array}$ & $\begin{array}{l}41.41^{* * *} \\
(14.095)\end{array}$ & $\begin{array}{c}-17.71 \\
(75.373)\end{array}$ \\
\hline Fixed $*$ Medium & $\begin{array}{c}0.05 \\
(0.097)\end{array}$ & $\begin{array}{c}39.05 \\
(38.874)\end{array}$ & $\begin{array}{c}614.96^{*} \\
(363.773)\end{array}$ & $\begin{array}{l}874.30^{* * *} \\
(267.884)\end{array}$ & $\begin{array}{c}29.15 \\
(55.345)\end{array}$ & $\begin{array}{l}28.20^{* *} \\
(14.180)\end{array}$ & $\begin{array}{c}101.22 \\
(77.715)\end{array}$ \\
\hline Fixed $*$ High & $\begin{array}{c}0.05 \\
(0.100)\end{array}$ & $\begin{array}{c}7.06 \\
(39.747)\end{array}$ & $\begin{array}{c}490.17 \\
(326.302)\end{array}$ & $\begin{array}{l}774.99 * * * \\
(248.393)\end{array}$ & $\begin{array}{c}11.07 \\
(51.280)\end{array}$ & $\begin{array}{c}20.60 \\
(14.313)\end{array}$ & $\begin{array}{c}-90.81 \\
(63.314)\end{array}$ \\
\hline Flexible * Low & $\begin{array}{l}0.21^{* *} \\
(0.096)\end{array}$ & $\begin{array}{c}68.06^{*} \\
(34.807)\end{array}$ & $\begin{array}{l}939.82^{* * *} \\
(286.172)\end{array}$ & $\begin{array}{l}966.59^{* * *} \\
(215.430)\end{array}$ & $\begin{array}{c}78.59^{*} \\
(42.362)\end{array}$ & $\begin{array}{c}24.90^{*} \\
(14.882)\end{array}$ & $\begin{array}{c}66.76 \\
(73.566)\end{array}$ \\
\hline Flexible ${ }^{*}$ Medium & $\begin{array}{c}0.17 \\
(0.108)\end{array}$ & $\begin{array}{c}27.46 \\
(43.234)\end{array}$ & $\begin{array}{c}84.52 \\
(372.727)\end{array}$ & $\begin{array}{c}419.22 \\
(300.658)\end{array}$ & $\begin{array}{c}11.86 \\
(61.472)\end{array}$ & $\begin{array}{c}28.63^{*} \\
(17.046)\end{array}$ & $\begin{array}{l}171.63^{*} \\
(92.894)\end{array}$ \\
\hline Flexible * High & $\begin{array}{c}0.15^{*} \\
(0.084)\end{array}$ & $\begin{array}{c}32.28 \\
(33.035)\end{array}$ & $\begin{array}{c}562.25^{*} \\
(330.432)\end{array}$ & $\begin{array}{c}433.71^{*} \\
(228.195)\end{array}$ & $\begin{array}{c}85.23^{*} \\
(44.986)\end{array}$ & $\begin{array}{l}30.91^{* *} \\
(14.430)\end{array}$ & $\begin{array}{c}-13.76 \\
(62.511)\end{array}$ \\
\hline Observations & 3608 & 3608 & 3608 & 3608 & 3608 & 3608 & 3608 \\
\hline Test: Fixed coefficients equal & 0.004 & 0.122 & 0.366 & 0.772 & 0.389 & 0.612 & 0.150 \\
\hline Test: Flexible coefficients equal & 0.918 & 0.700 & 0.210 & 0.194 & 0.601 & 0.960 & 0.227 \\
\hline Test: Diff-in-diff (high vs low tericle) & 0.022 & 0.219 & 0.618 & 0.511 & 0.209 & 0.268 & 0.952 \\
\hline
\end{tabular}

Note: In this table we investigate heterogeneous treatment effects using local average treatment effect estimates, where we instrument take-up with assignment to either of the two treatment contracts (fixed- or flexible-repayment), and interact with dummies for each of the three baseline heterogeneity terciles (where 'Low', 'Medium' and 'High' refers to individuals who were in the bottom, middle and highest terciles of the particular heterogeneity variable, as done in the equivalent table for the ITT results). The omitted category represents individuals in the control group from the lowest tercile. Standard errors, reported below each coefficient in parenthesis, allow for clustering at the level of the individual. We denote significance using $*$ for $10 \%, * *$ for $5 \%$ and $* * *$ for $1 \%$. In the bottom three rows of the table, we report p-values for three null hypotheses: (i) the null hypothesis that the effect of take-up of the fixed contract is equal across three terciles; (ii) the null hypothesis that the effect of take-up of the flexible contract is equal across terciles; and (iii) a difference-in-difference test: testing the null that the difference in outcomes between the lowest and highest tercile was the same across the two assignment groups. 
Table A.46: Outcome heterogeneity by baseline time-preference tercile: LATE

\begin{tabular}{|c|c|c|c|c|c|c|c|}
\hline & $\begin{array}{c}\text { (1) } \\
\text { Number of } \\
\text { businesses }\end{array}$ & $\begin{array}{l}\text { (2) } \\
\text { Business } \\
\text { profits }\end{array}$ & $\begin{array}{l}\text { (3) } \\
\text { Business } \\
\text { assets }\end{array}$ & $\begin{array}{c}(4) \\
\text { Fixed } \\
\text { assets }\end{array}$ & $\begin{array}{c}(5) \\
\text { Household } \\
\text { income }\end{array}$ & $\begin{array}{c}(6) \\
\text { Household } \\
\text { consumption }\end{array}$ & $\begin{array}{c}\text { (7) } \\
\text { Household } \\
\text { savings }\end{array}$ \\
\hline Medium & $\begin{array}{c}-0.01 \\
(0.069)\end{array}$ & $\begin{array}{c}-12.87 \\
(24.271)\end{array}$ & $\begin{array}{l}-327.20^{*} \\
(193.801)\end{array}$ & $\begin{array}{c}-256.08^{*} \\
(152.267)\end{array}$ & $\begin{array}{c}-1.64 \\
(30.988)\end{array}$ & $\begin{array}{l}22.06^{* *} \\
(11.019)\end{array}$ & $\begin{array}{c}-55.14 \\
(43.295)\end{array}$ \\
\hline High & $\begin{array}{c}0.06 \\
(0.056)\end{array}$ & $\begin{array}{c}2.67 \\
(20.042)\end{array}$ & $\begin{array}{l}-322.83^{*} \\
(187.230)\end{array}$ & $\begin{array}{l}-297.27^{* *} \\
(135.241)\end{array}$ & $\begin{array}{c}9.05 \\
(24.488)\end{array}$ & $\begin{array}{c}10.24 \\
(8.489)\end{array}$ & $\begin{array}{c}-42.81 \\
(44.344)\end{array}$ \\
\hline Fixed * Low & $\begin{array}{l}0.28^{* * * *} \\
(0.097)\end{array}$ & $\begin{array}{c}49.95 \\
(35.955)\end{array}$ & $\begin{array}{c}130.36 \\
(351.793)\end{array}$ & $\begin{array}{l}554.36^{* *} \\
(265.363)\end{array}$ & $\begin{array}{c}80.77^{*} \\
(46.712)\end{array}$ & $\begin{array}{l}57.04^{* * * *} \\
(16.922)\end{array}$ & $\begin{array}{c}-61.70 \\
(86.079)\end{array}$ \\
\hline Fixed ${ }^{*}$ Medium & $\begin{array}{l}0.47^{* * * *} \\
(0.130)\end{array}$ & $\begin{array}{c}127.29^{* * *} \\
(48.203)\end{array}$ & $\begin{array}{c}1569.73^{* * *} \\
(471.762)\end{array}$ & $\begin{array}{l}1446.10^{* * * *} \\
(401.805)\end{array}$ & $\begin{array}{c}74.76 \\
(67.297)\end{array}$ & $\begin{array}{c}-3.21 \\
(19.522)\end{array}$ & $\begin{array}{c}-19.49 \\
(93.881)\end{array}$ \\
\hline Fixed $*$ High & $\begin{array}{c}0.07 \\
(0.084)\end{array}$ & $\begin{array}{c}35.42 \\
(31.821)\end{array}$ & $\begin{array}{l}1007.16^{* * *} \\
(283.695)\end{array}$ & $\begin{array}{l}990.02^{* * * *} \\
(202.763)\end{array}$ & $\begin{array}{c}24.83 \\
(39.730)\end{array}$ & $\begin{array}{l}29.79^{* * *} \\
(11.213)\end{array}$ & $\begin{array}{c}35.68 \\
(63.100)\end{array}$ \\
\hline Flexible * Low & $\begin{array}{l}0.23^{* *} \\
(0.099)\end{array}$ & $\begin{array}{l}75.09 * * \\
(37.693)\end{array}$ & $\begin{array}{c}735.28^{*} \\
(403.781)\end{array}$ & $\begin{array}{l}573.18^{* *} \\
(259.286)\end{array}$ & $\begin{array}{l}109.12^{* *} \\
(52.593)\end{array}$ & $\begin{array}{c}30.74^{*} \\
(17.718)\end{array}$ & $\begin{array}{c}-65.41 \\
(79.191)\end{array}$ \\
\hline Flexible * Medium & $\begin{array}{l}0.29^{* * * *} \\
(0.109)\end{array}$ & $\begin{array}{c}71.05^{*} \\
(39.751)\end{array}$ & $\begin{array}{l}743.68^{* *} \\
(311.213)\end{array}$ & $\begin{array}{l}670.77^{* * * *} \\
(256.130)\end{array}$ & $\begin{array}{c}95.66^{*} \\
(51.948)\end{array}$ & $\begin{array}{c}13.87 \\
(16.322)\end{array}$ & $\begin{array}{c}88.31 \\
(76.863)\end{array}$ \\
\hline Flexible * High & $\begin{array}{c}0.08 \\
(0.092)\end{array}$ & $\begin{array}{c}13.03 \\
(35.367)\end{array}$ & $\begin{array}{c}534.93^{*} \\
(302.162)\end{array}$ & $\begin{array}{l}759.75^{* * *} \\
(212.061)\end{array}$ & $\begin{array}{c}18.99 \\
(45.628)\end{array}$ & $\begin{array}{c}32.79^{* *} \\
(13.424)\end{array}$ & $\begin{array}{l}145.08^{*} \\
(84.540)\end{array}$ \\
\hline Observations & 3608 & 3608 & 3608 & 3608 & 3608 & 3608 & 3608 \\
\hline Test: Fixed coefficients equal & 0.033 & 0.304 & 0.033 & 0.170 & 0.630 & 0.090 & 0.677 \\
\hline Test: Flexible coefficients equal & 0.302 & 0.435 & 0.879 & 0.873 & 0.410 & 0.644 & 0.195 \\
\hline Test: Diff-in-diff (high vs low tericle) & 0.676 & 0.371 & 0.046 & 0.505 & 0.661 & 0.254 & 0.381 \\
\hline
\end{tabular}

Note: In this table we investigate heterogeneous treatment effects using local average treatment effect estimates, where we instrument take-up with assignment to either of the two treatment contracts (fixed- or flexible-repayment), and interact with dummies for each of the three baseline heterogeneity terciles (where 'Low', 'Medium' and 'High' refers to individuals who were in the bottom, middle and highest terciles of the particular heterogeneity variable, as done in the equivalent table for the ITT results). The omitted category represents individuals in the control group from the lowest tercile. Standard errors, reported below each coefficient in parenthesis, allow for clustering at the level of the individual. We denote significance using $*$ for $10 \%, * *$ for $5 \%$ and $* * *$ for $1 \%$. In the bottom three rows of the table, we report p-values for three null hypotheses: (i) the null hypothesis that the effect of take-up of the fixed contract is equal across three terciles; (ii) the null hypothesis that the effect of take-up of the flexible contract is equal across terciles; and (iii) a difference-in-difference test: testing the null that the difference in outcomes between the lowest and highest tercile was the same across the two assignment groups. 


\section{Table A.47: Outcome heterogeneity by baseline management tercile: LATE}

\begin{tabular}{|c|c|c|c|c|c|c|c|}
\hline & $\begin{array}{c}\text { (1) } \\
\text { Number of } \\
\text { businesses }\end{array}$ & $\begin{array}{c}(2) \\
\text { Business } \\
\text { profits }\end{array}$ & $\begin{array}{c}\text { (3) } \\
\text { Business } \\
\text { assets }\end{array}$ & $\begin{array}{c}(4) \\
\text { Fixed } \\
\text { assets }\end{array}$ & $\begin{array}{l}\text { (5) } \\
\text { Household } \\
\text { income }\end{array}$ & $\begin{array}{c}(6) \\
\text { Household } \\
\text { consumption }\end{array}$ & $\begin{array}{c}\text { (7) } \\
\text { Household } \\
\text { savings }\end{array}$ \\
\hline Medium & $\begin{array}{c}-0.01 \\
(0.059)\end{array}$ & $\begin{array}{c}13.84 \\
(20.806)\end{array}$ & $\begin{array}{c}112.92 \\
(183.016)\end{array}$ & $\begin{array}{c}41.93 \\
(137.438)\end{array}$ & $\begin{array}{c}13.90 \\
(25.894)\end{array}$ & $\begin{array}{c}13.32 \\
(8.681)\end{array}$ & $\begin{array}{c}41.81 \\
(32.891)\end{array}$ \\
\hline High & $\begin{array}{c}-0.01 \\
(0.059)\end{array}$ & $\begin{array}{c}26.57 \\
(22.848)\end{array}$ & $\begin{array}{c}-12.81 \\
(208.339)\end{array}$ & $\begin{array}{c}19.00 \\
(143.321)\end{array}$ & $\begin{array}{c}46.75 \\
(29.215)\end{array}$ & $\begin{array}{l}15.92^{*} \\
(9.411)\end{array}$ & $\begin{array}{c}-0.64 \\
(41.819)\end{array}$ \\
\hline Fixed * Low & $\begin{array}{l}0.31^{* * *} \\
(0.113)\end{array}$ & $\begin{array}{c}91.70^{* *} \\
(39.085)\end{array}$ & $\begin{array}{c}1250.29^{* * *} \\
(384.630)\end{array}$ & $\begin{array}{c}1224.88^{* * *} \\
(318.340)\end{array}$ & $\begin{array}{c}60.20 \\
(51.774)\end{array}$ & $\begin{array}{l}67.11^{* * *} \\
(18.916)\end{array}$ & $\begin{array}{c}-18.05 \\
(64.756)\end{array}$ \\
\hline Fixed $*$ Medium & $\begin{array}{l}0.19^{* * *} \\
(0.086)\end{array}$ & $\begin{array}{c}65.94^{* *} \\
(32.991)\end{array}$ & $\begin{array}{l}753.94^{* * *} \\
(285.145)\end{array}$ & $\begin{array}{l}896.93^{* * *} \\
(213.746)\end{array}$ & $\begin{array}{c}65.62^{*} \\
(39.813)\end{array}$ & $\begin{array}{c}15.51 \\
(11.135)\end{array}$ & $\begin{array}{c}-86.12 \\
(54.754)\end{array}$ \\
\hline Fixed $*$ High & $\begin{array}{c}0.18^{*} \\
(0.101)\end{array}$ & $\begin{array}{c}22.00 \\
(42.020)\end{array}$ & $\begin{array}{c}490.15 \\
(370.291)\end{array}$ & $\begin{array}{c}649.35^{* *} \\
(262.201)\end{array}$ & $\begin{array}{c}33.43 \\
(55.146)\end{array}$ & $\begin{array}{c}16.00 \\
(15.299)\end{array}$ & $\begin{array}{c}101.92 \\
(99.984)\end{array}$ \\
\hline Flexible * Low & $\begin{array}{c}0.03 \\
(0.112)\end{array}$ & $\begin{array}{c}23.87 \\
(37.020)\end{array}$ & $\begin{array}{l}775.46^{* * *} \\
(348.301)\end{array}$ & $\begin{array}{l}589.21^{* *} \\
(249.588)\end{array}$ & $\begin{array}{c}53.30 \\
(45.680)\end{array}$ & $\begin{array}{c}33.47^{*} \\
(17.645)\end{array}$ & $\begin{array}{c}0.97 \\
(54.550)\end{array}$ \\
\hline Flexible * Medium & $\begin{array}{l}0.34^{* * *} \\
(0.108)\end{array}$ & $\begin{array}{c}106.58^{* * *} \\
(39.598)\end{array}$ & $\begin{array}{c}505.59 \\
(311.330)\end{array}$ & $\begin{array}{c}645.62^{* *} \\
(258.706)\end{array}$ & $\begin{array}{c}155.28^{* * *} \\
(49.368)\end{array}$ & $\begin{array}{c}32.18^{* *} \\
(15.284)\end{array}$ & $\begin{array}{c}114.85 \\
(83.582)\end{array}$ \\
\hline Flexible * High & $\begin{array}{l}0.22^{* * *} \\
(0.078)\end{array}$ & $\begin{array}{c}20.96 \\
(32.081)\end{array}$ & $\begin{array}{c}615.09^{* *} \\
(289.234)\end{array}$ & $\begin{array}{l}740.49^{* * *} \\
(201.342)\end{array}$ & $\begin{array}{c}0.55 \\
(44.709)\end{array}$ & $\begin{array}{c}22.93 \\
(14.228)\end{array}$ & $\begin{array}{c}78.51 \\
(89.890)\end{array}$ \\
\hline Observations & 3608 & 3608 & 3608 & 3608 & 3608 & 3608 & 3608 \\
\hline Test: Fixed coefficients equal & 0.634 & 0.497 & 0.378 & 0.408 & 0.893 & 0.060 & 0.262 \\
\hline Test: Flexible coefficients equal & 0.126 & 0.211 & 0.851 & 0.886 & 0.074 & 0.879 & 0.461 \\
\hline Test: Diff-in-diff (high vs low tericle) & 0.025 & 0.217 & 0.242 & 0.085 & 0.728 & 0.086 & 0.733 \\
\hline
\end{tabular}

Note: In this table we investigate heterogeneous treatment effects using local average treatment effect estimates, where we instrument take-up with assignment to either of the two treatment contracts (fixed- or flexible-repayment), and interact with dummies for each of the three baseline heterogeneity terciles (where 'Low', 'Medium' and 'High' refers to individuals who were in the bottom, middle and highest terciles of the particular heterogeneity variable, as done in the equivalent table for the ITT results). The omitted category represents individuals in the control group from the lowest tercile. Standard errors, reported below each coefficient in parenthesis, allow for clustering at the level of the individual. We denote significance using $*$ for $10 \%, * *$ for $5 \%$ and $* * *$ for $1 \%$. In the bottom three rows of the table, we report p-values for three null hypotheses: (i) the null hypothesis that the effect of take-up of the fixed contract is equal across three terciles; (ii) the null hypothesis that the effect of take-up of the flexible contract is equal across terciles; and (iii) a difference-in-difference test: testing the null that the difference in outcomes between the lowest and highest tercile was the same across the two assignment groups. 
Table A.48: Outcome heterogeneity by baseline numeracy tercile: LATE

\begin{tabular}{|c|c|c|c|c|c|c|c|}
\hline & $\begin{array}{c}\text { (1) } \\
\text { Number of } \\
\text { businesses }\end{array}$ & $\begin{array}{l}\text { (2) } \\
\text { Business } \\
\text { profits }\end{array}$ & $\begin{array}{l}\text { (3) } \\
\text { Business } \\
\text { assets }\end{array}$ & $\begin{array}{c}(4) \\
\text { Fixed } \\
\text { assets }\end{array}$ & $\begin{array}{l}(5) \\
\text { Household } \\
\text { income }\end{array}$ & $\begin{array}{c}\text { (6) } \\
\text { Household } \\
\text { consumption }\end{array}$ & $\begin{array}{c}\text { (7) } \\
\text { Household } \\
\text { savings }\end{array}$ \\
\hline Medium & $\begin{array}{c}-0.02 \\
(0.062)\end{array}$ & $\begin{array}{c}-14.82 \\
(22.675)\end{array}$ & $\begin{array}{c}251.74 \\
(189.056)\end{array}$ & $\begin{array}{c}213.53 \\
(132.990)\end{array}$ & $\begin{array}{c}-20.28 \\
(28.821)\end{array}$ & $\begin{array}{l}17.37^{*} \\
(9.460)\end{array}$ & $\begin{array}{c}2.62 \\
(53.553)\end{array}$ \\
\hline High & $\begin{array}{c}0.03 \\
(0.060)\end{array}$ & $\begin{array}{c}5.44 \\
(21.479)\end{array}$ & $\begin{array}{l}389.65^{* *} \\
(171.896)\end{array}$ & $\begin{array}{l}262.01^{* *} \\
(121.337)\end{array}$ & $\begin{array}{c}-2.49 \\
(27.051)\end{array}$ & $\begin{array}{l}22.75^{* *} \\
(9.361)\end{array}$ & $\begin{array}{c}-34.87 \\
(39.099)\end{array}$ \\
\hline Fixed $*$ Low & $\begin{array}{l}0.20^{* *} \\
(0.086)\end{array}$ & $\begin{array}{c}50.75 \\
(33.046)\end{array}$ & $\begin{array}{c}1365.99^{* * *} \\
(315.373)\end{array}$ & $\begin{array}{c}1255.38^{* * *} \\
(246.489)\end{array}$ & $\begin{array}{c}47.50 \\
(43.693)\end{array}$ & $\begin{array}{l}63.89^{* * *} \\
(15.372)\end{array}$ & $\begin{array}{c}-92.35 \\
(64.628)\end{array}$ \\
\hline Fixed ${ }^{*}$ Medium & $\begin{array}{l}0.34^{* * *} \\
(0.120)\end{array}$ & $\begin{array}{l}89.93^{* *} \\
(43.462)\end{array}$ & $\begin{array}{c}710.75^{*} \\
(417.061)\end{array}$ & $\begin{array}{l}943.76^{* * *} \\
(300.042)\end{array}$ & $\begin{array}{c}38.99 \\
(54.767)\end{array}$ & $\begin{array}{c}3.34 \\
(15.959)\end{array}$ & $\begin{array}{c}-20.00 \\
(98.919)\end{array}$ \\
\hline Fixed $*$ High & $\begin{array}{c}0.17^{*} \\
(0.099)\end{array}$ & $\begin{array}{c}48.83 \\
(36.911)\end{array}$ & $\begin{array}{c}340.39 \\
(309.482)\end{array}$ & $\begin{array}{l}568.08^{* *} \\
(235.814)\end{array}$ & $\begin{array}{c}71.77 \\
(45.648)\end{array}$ & $\begin{array}{c}18.00 \\
(13.908)\end{array}$ & $\begin{array}{c}81.67 \\
(79.250)\end{array}$ \\
\hline Flexible * Low & $\begin{array}{c}0.15 \\
(0.096)\end{array}$ & $\begin{array}{c}49.57 \\
(36.239)\end{array}$ & $\begin{array}{c}1187.36^{* * *} \\
(314.395)\end{array}$ & $\begin{array}{l}944.46^{* * *} \\
(252.457)\end{array}$ & $\begin{array}{c}46.50 \\
(49.606)\end{array}$ & $\begin{array}{l}51.61^{* * *} \\
(16.803)\end{array}$ & $\begin{array}{c}38.51 \\
(78.317)\end{array}$ \\
\hline Flexible ${ }^{*}$ Medium & $\begin{array}{l}0.27^{* *} \\
(0.132)\end{array}$ & $\begin{array}{c}75.17 \\
(48.792)\end{array}$ & $\begin{array}{c}528.59 \\
(401.338)\end{array}$ & $\begin{array}{c}318.46 \\
(280.481)\end{array}$ & $\begin{array}{c}102.77 \\
(63.627)\end{array}$ & $\begin{array}{c}27.37 \\
(19.129)\end{array}$ & $\begin{array}{c}-21.54 \\
(87.455)\end{array}$ \\
\hline Flexible * High & $\begin{array}{l}0.17^{* *} \\
(0.084)\end{array}$ & $\begin{array}{c}31.59 \\
(31.034)\end{array}$ & $\begin{array}{c}222.05 \\
(248.484)\end{array}$ & $\begin{array}{l}578.62^{* * * *} \\
(180.422)\end{array}$ & $\begin{array}{c}63.88 \\
(39.534)\end{array}$ & $\begin{array}{c}9.67 \\
(12.222)\end{array}$ & $\begin{array}{l}128.51^{*} \\
(74.891)\end{array}$ \\
\hline Observations & 3608 & 3608 & 3608 & 3608 & 3608 & 3608 & 3608 \\
\hline Test: Fixed coefficients equal & 0.551 & 0.725 & 0.072 & 0.148 & 0.887 & 0.020 & 0.238 \\
\hline Test: Flexible coefficients equal & 0.735 & 0.749 & 0.062 & 0.236 & 0.788 & 0.146 & 0.413 \\
\hline Test: Diff-in-diff (high vs low tericle) & 0.734 & 0.746 & 0.899 & 0.407 & 0.919 & 0.859 & 0.523 \\
\hline
\end{tabular}

Note: In this table we investigate heterogeneous treatment effects using local average treatment effect estimates, where we instrument take-up with assignment to either of the two treatment contracts (fixed- or flexible-repayment), and interact with dummies for each of the three baseline heterogeneity terciles (where 'Low', 'Medium' and 'High' refers to individuals who were in the bottom, middle and highest terciles of the particular heterogeneity variable, as done in the equivalent table for the ITT results). The omitted category represents individuals in the control group from the lowest tercile. Standard errors, reported below each coefficient in parenthesis, allow for clustering at the level of the individual. We denote significance using $*$ for $10 \%, * *$ for $5 \%$ and $* * *$ for $1 \%$. In the bottom three rows of the table, we report p-values for three null hypotheses: (i) the null hypothesis that the effect of take-up of the fixed contract is equal across three terciles; (ii) the null hypothesis that the effect of take-up of the flexible contract is equal across terciles; and (iii) a difference-in-difference test: testing the null that the difference in outcomes between the lowest and highest tercile was the same across the two assignment groups. 


\title{
N Script describing the fixed-repayment contract
}

\author{
English translation of the script for demonstration of fixed-repayment contract \\ Introduction
}

In this meeting, we are going to describe a type of contract that Akhuwat would like to offer to some of its selected clients, like you. This contract is designed to help entrepreneurs like you purchase an asset for their business. If you are offered this contract, you are allowed to use the money to buy a large asset for your business, such as a sewing machine if you make clothes, or for example a rickshaw if you are a rickshaw driver, or for example a welding plant if you do welding, or for example a molding machine if that is your business. You are not allowed to use it to purchase raw materials or inventories or stock that you then sell to people: for example, if you were a tailor, then you would not be allowed to use the money to buy thread or clothes. The benefit of this new contract is that it allows you to access finance for a larger amount than is currently available from Akhuwat,

The purpose of this meeting is to describe the contract to you. We would like you to understand how the contract works. We will describe the contract and then we will ask you questions to see if you have understood the contract. Please do not tell us now in this meeting about if you are interested in the contract or not. After the meeting, on another day, an Akhuwat member of staff will visit you to ask if you would be interested in this contract. The purpose of the group meeting is just to explain the contract and check that you have understood how it works. Please feel free to ask us as many questions as you want about the contract. It is very important that you understand how this contracts works: what payments you have to make and what happens if you do not make the payments

Does anybody have any question?

\section{Ali the Tailor}

We will explain how the contract works using the hypothetical story of a business person called Ali. Ali lives and works in Township, Lahore. Ali owns and manages a small tailor business, where he stitches clothes by hand. Ali's business is successful, but he would like to expand his business. Ali thinks that if he buys a sewing machine, his profits will increase a lot. Ali has a small amount of savings, $10,000 \mathrm{Rs}$, but it is not enough to buy a sewing machine, which costs 100,000

Akhuwat can help Ali. Ali has 10,000 Rs of savings. The sewing machine costs 100,000 Rs. If Akhuwat contributes 90,000 Rs, then Akhuwat and Ali can buy the asset together. Akhuwat and Ali will then own the asset together. This type of partnership, or joint ownership, is called "shirakat." Akhuwat will own $90 \%$ of the sewing machine, and Ali will own $10 \%$ of the sewing machine.

But Akhuwat does not need to use the sewing machine. Ali does need to use the sewing machine, for his business. So Akhuwat and Ali make an agreement. Ali will use the sewing machine for his business, and he will pay rent to Akhuwat every month because he is the only one who will benefit from using it. So Ali will pay Akhuwat rent, or "kirayah" Ali will also be responsible for all daily repairs of the asset, because he is the one that is using it, but if there is a natural disaster then Akhuwat will share in the risk of this with Ali.

\section{ENUMERATOR: PLEASE BE VERY CAREFUL IN EXPLAINING THE NUMBERS NOW:}

The amount of rent that Ali will pay to Akhuwat is based on the percentage of the asset that Akhuwat owns. Imagine that Akhuwat and Ali agree on a rental rate of $1 \%$ per month for the sewing machine." $1 \%$ of 100,000 Rs is 1,000 Rs. Because Akhuwat owns $90 \%$ of the sewing machine, Akhuwat is entitled to $90 \%$ of 1,000 Rs, which is 900 Rs. So Ali must pay Akhuwat rent of $900 \mathrm{Rs}$ at the end of the first month.

ENUMERATOR: Illustrate all of this.

Does everybody understand? Does anybody have any questions? 
As mentioned, Akhuwat owns $90 \%$ of the sewing machine, and Ali owns $10 \%$. However, this ownership share can change. Ali can purchase some of Akhuwat's ownership share. If Ali gives Akhuwat 5,000 Rs, then he can purchase 5\% of Akhuwat's ownership share in the sewing machine. This would increase Ali's ownership share of the asset by $5 \%$, and decrease Akhuwat's share by $5 \%$. Ali's ownership share would increase from $10 \%$ before to $15 \%$ now, and Akhuwat's ownership share would decrease from $90 \%$ before to $85 \%$ now.

The benefit of this to Ali is that he now pays less rent. Before, when his ownership share of the sewing machine was only $10 \%$, and Akhuwat's ownership share was $90 \%$, Ali had to pay rent on Akhuwat's share of 900 Rs per month. But after he repurchased $5 \%$ from Akhuwat's share, Ali now owns $15 \%$ of the asset, and Akhuwat owns $85 \%$, so the rent he pays will decrease to $850 \mathrm{Rs}$ per month.

Does everybody understand? Does anybody have any questions?

We have now explained the basic idea about this new contract that Akhuwat is interested in offering to some of its selected clients, using the example of Ali. The main ideas that we discussed were:

1) Joint-ownership of the sewing machine between Akhuwat and Ali, or 'shiraakat'

2) A payment of rent or "kirayah" from Ali to Akhuwat, because Ali is using the asset and Akhuwat owns some of the sewing machine.

3) A reduction in the amount of rent that Ali has to pay when he repurchases some of Akhuwat's ownership share in the sewing machine.

We will now describe the contract that Akhuwat is interested in offering to some of its clients, using the same ideas as the example with Ali. Please do not tell us now in this meeting about if you are interested in the contract or not. After the meeting, on another day, an Akhuwat member of staff will visit you to ask if you would be interested in this contract.

\section{Describing the Contract: The Fixed-Repayment Contract}

The contract is a "fixed-repayment" contract. We will again illustrate it with the example of Ali the tailor and the sewing machine.

In this fixed-repayment contract, Ali and Akhuwat make an agreement for 18 months. The agreement says that, every month, Ali has to buy back $5 \%$ of Akhuwat's ownership share in the sewing machine. That means that Ali has to pay 5,000 Rs to Akhuwat every month, for 18 months. Every time Ali pays 5,000 to Akhuwat, his ownership share of the sewing machine increases by $5 \%$, and Akhuwat's ownership share decreases by $5 \%$. We can see what this looks like using a table:

Fixed Payment Contract

\begin{tabular}{|c|c|c|}
\hline Start of Month & Akhuwat & Ali \\
\hline 1 & $90 \%$ & $10 \%$ \\
\hline 2 & $85 \%$ & $15 \%$ \\
\hline 3 & $80 \%$ & $20 \%$ \\
\hline
\end{tabular}




\begin{tabular}{|c|c|c|}
\hline 4 & $75 \%$ & $25 \%$ \\
\hline 5 & $70 \%$ & $30 \%$ \\
\hline 6 & $65 \%$ & $35 \%$ \\
\hline 7 & $60 \%$ & $40 \%$ \\
\hline 8 & $55 \%$ & $45 \%$ \\
\hline 9 & $50 \%$ & $50 \%$ \\
\hline 10 & $45 \%$ & $55 \%$ \\
\hline 11 & $40 \%$ & $60 \%$ \\
\hline 12 & $35 \%$ & $65 \%$ \\
\hline 13 & $30 \%$ & $70 \%$ \\
\hline 14 & $25 \%$ & $75 \%$ \\
\hline 15 & $20 \%$ & $80 \%$ \\
\hline 16 & $15 \%$ & $85 \%$ \\
\hline 17 & $10 \%$ & $90 \%$ \\
\hline 18 & $5 \%$ & $95 \%$ \\
\hline End & $0 \%$ & $0 \%$ \\
\hline
\end{tabular}

ENUMERATOR: Describe, slowly, how Akhuwat's ownership share decreases by $5 \%$ every month.

So this "fixed-repayment" agreement between Ali and Akuwat means that Ali must pay 5,000 every month to Akhuwat to purchase Akhuwat's ownership share in the asset. But during those 18 months, the asset will stay with Ali, and he will benefit from it, so he must also pay rent or "kirayah," like we described before. Because the rent payment that Ali pays to Akhuwat every month is dependent upon the ownership share, the rent amount will decrease each month as Akhuwat's ownership share decreases. We can see what this looks like using a table:

\begin{tabular}{|c|c|c|c|c|}
\hline Start of Month & Ali & Akhuwat & End of Month Rent & End of Month Payment \\
\hline 1 & 10 & 90 & 900 & 5000 \\
\hline 2 & 15 & 85 & 850 & 5000 \\
\hline 3 & 20 & 80 & 800 & 5000 \\
\hline 4 & 25 & 75 & 750 & 5000 \\
\hline 5 & 30 & 70 & 700 & 5000 \\
\hline 6 & 35 & 65 & 650 & 5000 \\
\hline 7 & 40 & 60 & 600 & 5000 \\
\hline
\end{tabular}




\begin{tabular}{|c|c|c|c|c|}
\hline 8 & 45 & 55 & 550 & 5000 \\
\hline 9 & 50 & 50 & 500 & 5000 \\
\hline 10 & 55 & 45 & 450 & 5000 \\
\hline 11 & 60 & 40 & 300 & 5000 \\
\hline 12 & 65 & 35 & 300 & 5000 \\
\hline 13 & 70 & 30 & 250 & 5000 \\
\hline 14 & 75 & 25 & 200 & 5000 \\
\hline 15 & 80 & 20 & 150 & 5000 \\
\hline 16 & 90 & 15 & 100 & 5000 \\
\hline 17 & 95 & 5 & 50 & 5000 \\
\hline 18 & $100 \%$ & $0 \%$ & 0 & 5000 \\
\hline End & & & & 0 \\
\hline
\end{tabular}

ENUMERATOR: Describe, slowly, how Ali's rental payment changes as the ownership share changes.

Does everybody understand how this fixed-repayment contract works? Does anybody have any questions?

\section{Missed Payments}

Now we will describe what happens if Ali misses any of the requirement payments in the contract.

If Ali cannot pay any of his required payments, then he has to make the payment in the next month. However, if he still cannot make the payment in the next month, then Ali has to agree that Akhuwat will repossess the sewing machine.

To help in this process - before this contract starts - Akhuwat and Ali will agree to have a "witness" to the contract, who will agree to assist in the process of delivering the sewing machine back to Akhuwat in this situation that we have described. But the "witness" will not have any obligation to make any payment if Ali misses a payment.

When Akhuwat repossesses the sewing machine, Akhuwat will sell the sewing machine in the market. The proceeds of the sale will be distributed in proportion to the ownership shares. For example, if the sewing machine was sold for $80,000 \mathrm{Rs}$ in the market, and Akhuwat and Ali's ownership share was $50 \%-50 \%$, then Akhuwat will keep 40,000 Rs and Ali will keep 40,000 Rs. After that, the contract between Ali and Akhuwat will be finished.

\section{Other}

Akhuwat has only a limited number of products that it can offer at the moment. The computer will decide who will be offered the product, using a random process (like drawing lots). We have no influence on the decision of the computer. The computer will decide randomly if you are selected to be offered the contract. Some, people will not be offered the contract. This will not take place today, but will take place after we have identified the asset that you would like to purchase.

Does everybody understand? Does anybody have any questions? 


\section{O Further details on the structural model}

\section{O.1 Introducing microfinance to the base model}

Allowing for an unconditional loan: The introduction of the new state variable requires an amendment to the value function:

$$
\begin{aligned}
& V_{m}\left(k_{t}, f_{t}, \varepsilon_{t}, \psi_{t}, x_{t}\right) \\
& =\left\{\begin{array}{ll}
\max _{k_{t+1}, f_{t+1}} \mathbb{E}_{\left(\varepsilon_{t+1}, \psi_{t+1}\right) \mid\left(\varepsilon_{t}, \psi_{t}\right)}\left[\frac{c_{t}^{1-1 / \gamma}}{1-1 / \gamma}+\beta \cdot V_{m}\left(k_{t+1}, f_{t+1}, \varepsilon_{t+1}, \psi_{t+1}, x_{t}+1\right)\right] \\
V_{n}\left(k_{t}, f_{t}, \varepsilon_{t}\right) & \text { if } x_{t} \leq X ;
\end{array}\right]
\end{aligned}
$$

That is, we treat the household as maximising discounted future consumption over the duration of the loan cycle, where the continuation value (after the cycle ends) is defined by the no-contract value function $V_{n}$.

Allowing for the asset financing contract: Alternatively, suppose that the household receives the asset financing contract, excluding the flexible-repayment option, and with a uniform asset amount of $\tilde{F}$. We model this scenario by (i) keeping the amended value function in equation 2' (because, as in the standard loan case, the household needs to optimise based upon its position in the cycle), (ii) by reverting to the initial restriction $f_{t} \geq 0$ (because the asset-finance contract is implemented as a direct injection of fixed capital, rather than as a relaxation of the financial constraint), and (iii) by amending equation 4:

$$
s_{t}=f_{t+1}-(1+r) \cdot f_{t}+ \begin{cases}\tilde{F} \cdot\left\{1 / 3+0.04 \times\left[1-0.15 \times\left(x_{t}-1\right)\right]\right\} & \text { if } x_{t}=1 \\ \tilde{F} \cdot\left\{1 / 5+0.04 \times\left[1-0.15 \times\left(x_{t}-1\right)\right]\right\} & \text { if } x_{t}>1\end{cases}
$$

Equation $4^{\prime}$ adjusts the earlier law of motion for $f_{t}$, by taking from the household a repayment to the MFI: this comprises an ownership purchase payment as well as a rental payment - and, in the initial period, also requires a $10 \%$ deposit. $^{5}$

\section{O.2 First-stage GMM estimation}

Denote the microenterprise value-added as $y_{i t}$. Following Blundell and Bond (2000), define $m_{i t}$ as the residual from a ' $\rho^{2}$-differenced' production function in logs:

$$
m_{i t} \equiv \ln y_{i t}-\left[\left(1-\rho^{2}\right) \cdot a+\rho^{2} \cdot \ln y_{i, t-1}+\alpha \cdot \ln k_{i t}-\alpha \rho^{2} \cdot \ln k_{i, t-1}\right] .
$$

\footnotetext{
5 The particular numbers used in equation $4{ }^{\prime}$ are specific to the particular implementation in our context including the representation, discussed shortly, that each time period comprises three calendar months.
} 
Then equations 2, 3 and 5 in the main paper together imply the following valid moment conditions:

$$
\begin{aligned}
\mathbb{E}\left(m_{i t}\right) & =0 ; \\
\mathbb{E}\left(m_{i t} \cdot \ln y_{i, t-1}\right) & =0 ; \\
\mathbb{E}\left(m_{i t} \cdot \ln k_{i, t-1}\right) & =0 ; \text { and } \\
\mathbb{E}\left(\sigma^{2}-m_{i t}^{2}\right) & =0 .
\end{aligned}
$$

Table A.49 shows the estimates obtained from our first-stage GMM estimation.

Table A.49: Structural estimates: First-stage GMM estimates

\begin{tabular}{ccc}
\hline PARAMETER & ESTIMATE & (S.E.) \\
\hline$\mu$ & 5.93 & $(0.12)$ \\
$\rho$ & 0.62 & $(0.03)$ \\
$\alpha$ & 0.16 & $(0.02)$ \\
$\sigma$ & 0.30 & $(0.01)$ \\
\hline
\end{tabular}

\section{O.3 Discretization and interpolation}

As discussed in the main paper, the state space for the no-contract model is $\left(k_{t}, f_{t}, \varepsilon_{t}, \psi_{t}\right)$. We discretize $k_{t}$ using 25 points (using a log-linear grid from US\$10 to US\$5000) and we discretize $f_{t}$ using 25 points (using a linear grid from the loan size to zero, and then to US\$3000). We discretize $\varepsilon_{t}$ using 5 points, using the usual method of Tauchen (1986). We solve the model at each of these points, in which we further discretize $k_{t+1}$ and $f_{t+1}$ and interpolate by linear approximation after transforming according to the inverse marginal utility of consumption (Blundell et al., 2016; Carroll, 2020). The model is specified and solved in discrete time; we treat each quarter as a different time period (thus we use six time points to solve for the 18-month contract horizon).

As discussed in the paper, we solve for $V_{n}$ (the no-contract case) by iterating to convergence on a Bellman equation. With the solution to $V_{n}$ in hand, we then solve for the two separate microfinance cases using backward induction. We then use these solutions for simulation; we do this by forming three Markov matrcies (one for the no-contract case, one for the standard loan case, and one for the fixed-repayment case), and then drawing from those matrices. For the initial distribution, we use the baseline joint distribution of $\left(k_{t}, f_{t}\right)$, and assume that this is independent of the initial distribution of $\varepsilon_{t}$. We use 2500 simulated observations, and we use the observed empirical take-up rates for both control and $\mathrm{T} 1$ groups.

\section{O.4 Simulated moments}

As discussed in the paper, we target Average Treatment Effects for fixed capital, microenterprise value-added and household consumption; we target these parameters at 
the three-month, six-month, 12-month, 18-month and 24-month follow-ups. This implies 15 moments in total. Denote by $s$ the vector of the 15 targeted moments in the real data; denote by $\tilde{\boldsymbol{s}}(\boldsymbol{\theta})$ the equivalent vector in the simulated data. We weight each of these moments by the inverse of the standard error from a regression on the T1 dummy (in an ANCOVA specification, partialling out strata dummies). Denote by $\boldsymbol{\Omega}_{s}$ a diagonal matrix recording these variances. Our Indirect Inference loss function is therefore formed as follows:

$$
(s-\tilde{s})^{\prime} \cdot \Omega_{s}^{-1} \cdot(s-\tilde{s}) .
$$

\section{O.5 Goodness of fit}

In Figure A.8, we show the real treatment effects (for fixed capital, value-added and consumption, at all follow-up waves), with a $95 \%$ confidence interval; we superimpose simulated treatment effects under model variants representing large non-convex capital adjustment costs $(\kappa=1500)$ and no such costs $(\kappa=0)$. The model with $\kappa=1500$ replicates large and persistent treatment effects on both fixed capital and enterprise valueadded. In contrast, the treatment effects cannot be replicated by the $\kappa=0$ version of the model; in that version, the control group is able to catch up quickly, both in terms of fixed capital and value-added.

Figure A.9 shows the goodness-of-fit for a large number of untargeted moments, both for the preferred model version with large non-convex adjustment costs $(\kappa=1500)$ and for the case without such costs $(\kappa=0)$. Specifically, we compare model predictions to data for fixed capital (both in levels and in first differences), for value-added (in levels and in differences) for household consumption (in levels and in differences) and for financial assets (in levels); we do this both for control and treatment groups, at the three-month, six-month, 12-month, 18-month and 24-month marks, and we map the 25th, 50th and 75th percentiles. The figure shows that the model fit is much better under $\kappa=1500$ than $\kappa=0$. In particular, under $\kappa=0$, the model predicts substantially more capital accumulation - both in control and treatment groups - than is actually observed.

In Figure A.10 we show the real and simulated moments for the control group; in Figure A.11, we show the same for treatment group 1. (In each case, we show the observed moment in black, with a 95\% confidence interval, and show the simulated moment in red.) In particular, our model replicates three characteristics of the data very closely. First, we fit very closely the distribution of fixed capital. This is true both in levels and in first differences. We replicate the important feature that (as noted, for example, in Levinsohn and Petrin (2003)) a large share of firms do not adjust their fixed capital from one period to the next. Second, we fit closely the pass-through of fixed capital into microenterprise value-added; this is illustrated by the tight fit on the value-added moments, both in levels and in differences. Third, we replicate the key causal chain discussed in our experimental results: when we compare treatment to control, we find an increase in fixed capital, which causes an increase (of the relevant magnitude) across the distribution of value-added, which then leads to an increase in household consumption. 


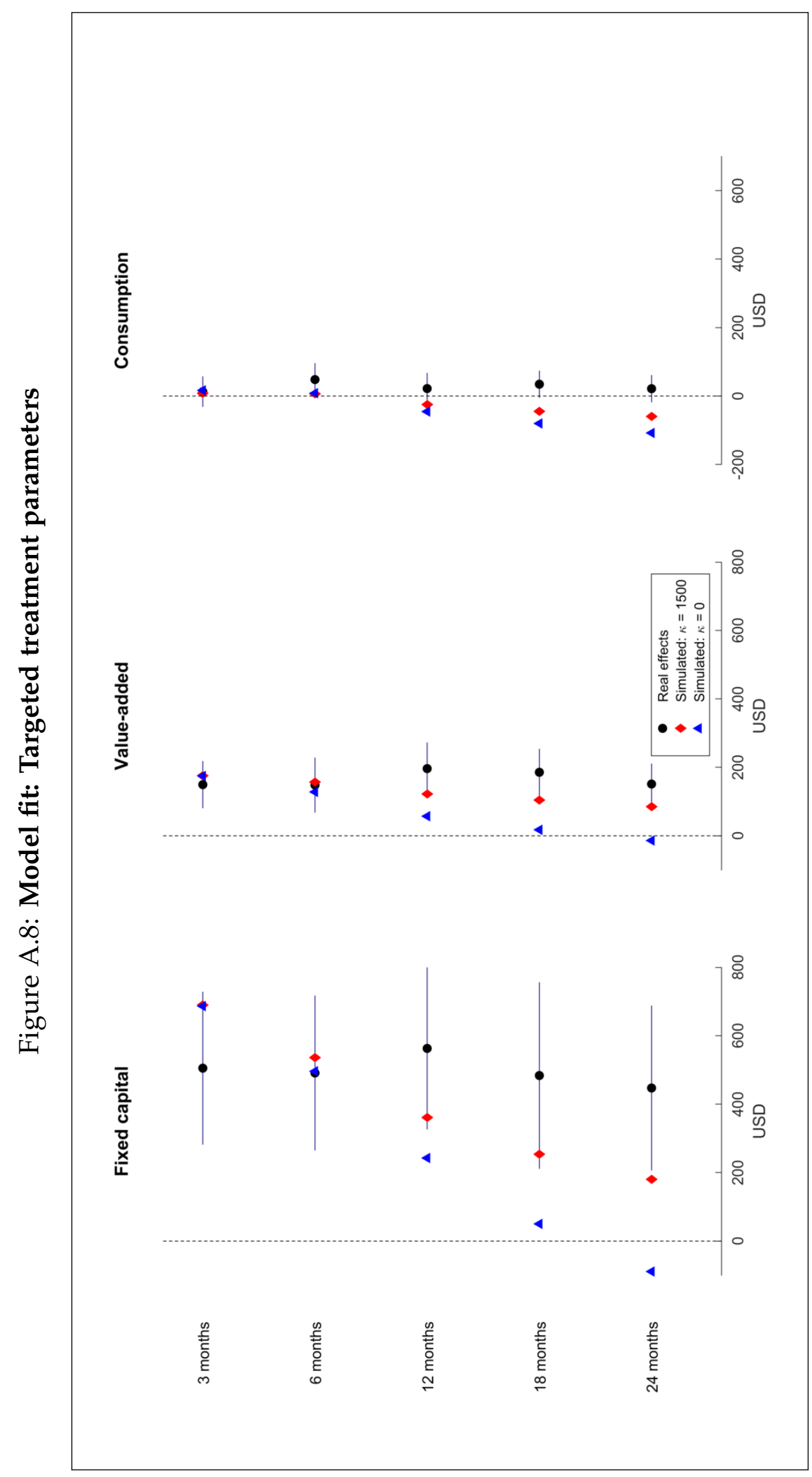


Figure A.9: Model fit: Untargeted quantile parameters

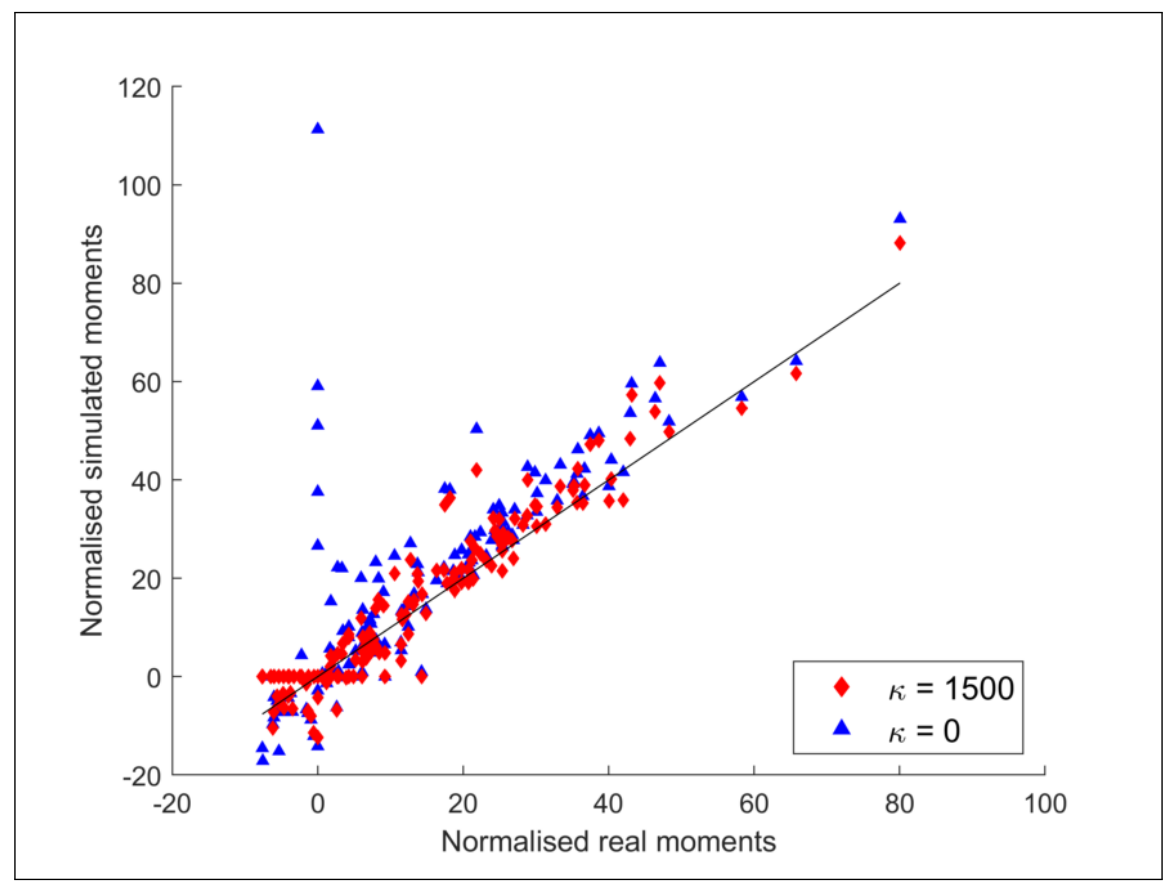




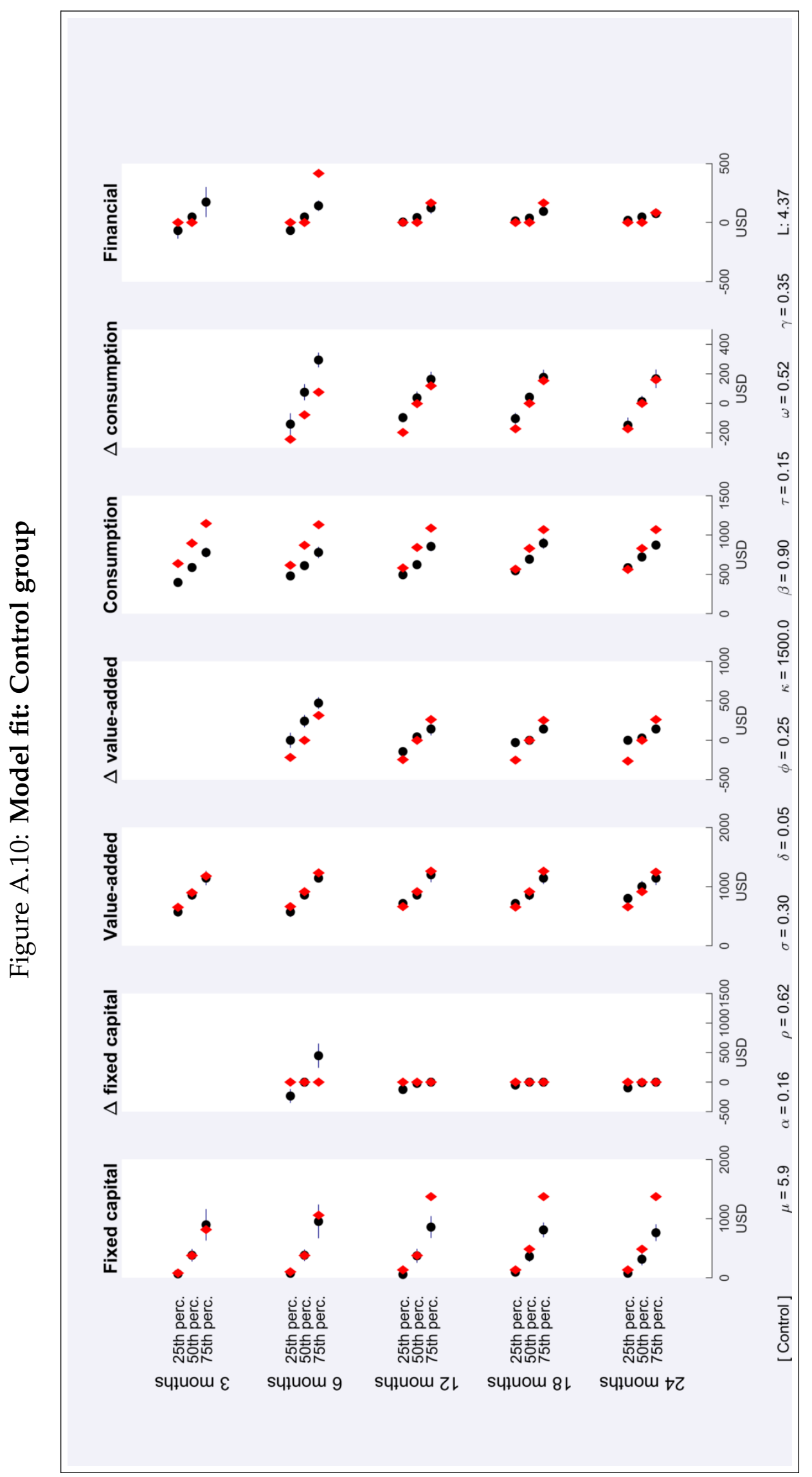




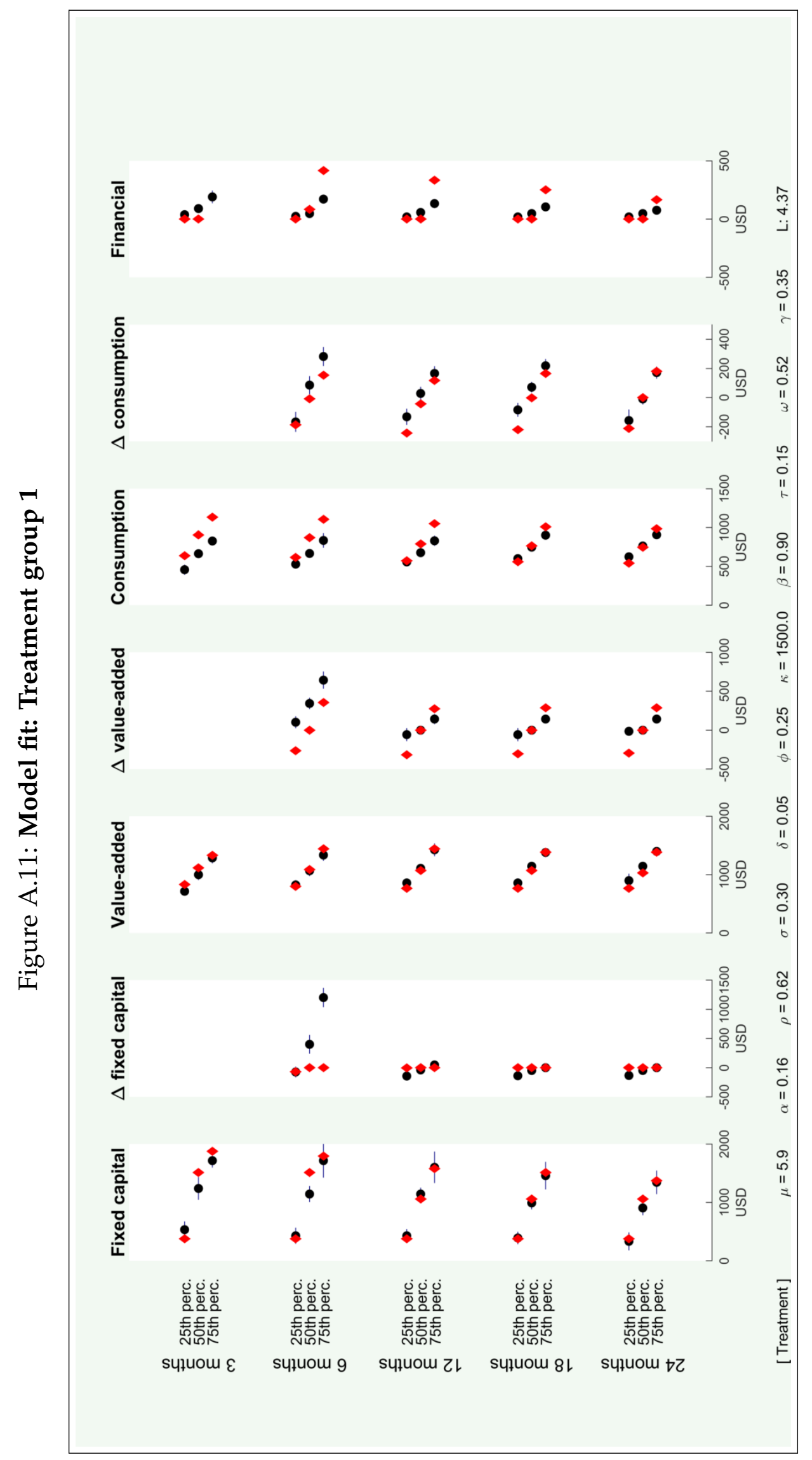




\section{O.6 Phase diagram}

Figure A.12 shows a phase diagram in $(k, f)$ space (illustrated for the no-contract case, and setting $(\varepsilon, \psi)=(0,1))$. We illustrate period-to-period transitions with arrows, and use larger red dots to indicate fixed points (that is, points that would be fixed if $(\varepsilon, \psi)=$ $(0,1)$ forever). The diagram shows that the non-convex capital adjustment costs generate a wide range of stable points; these cover a range of values of $k_{t+1}$, and each involves $f_{t+1} \approx 0$.

Figure A.12: Phase diagram in $(k, f)$ space

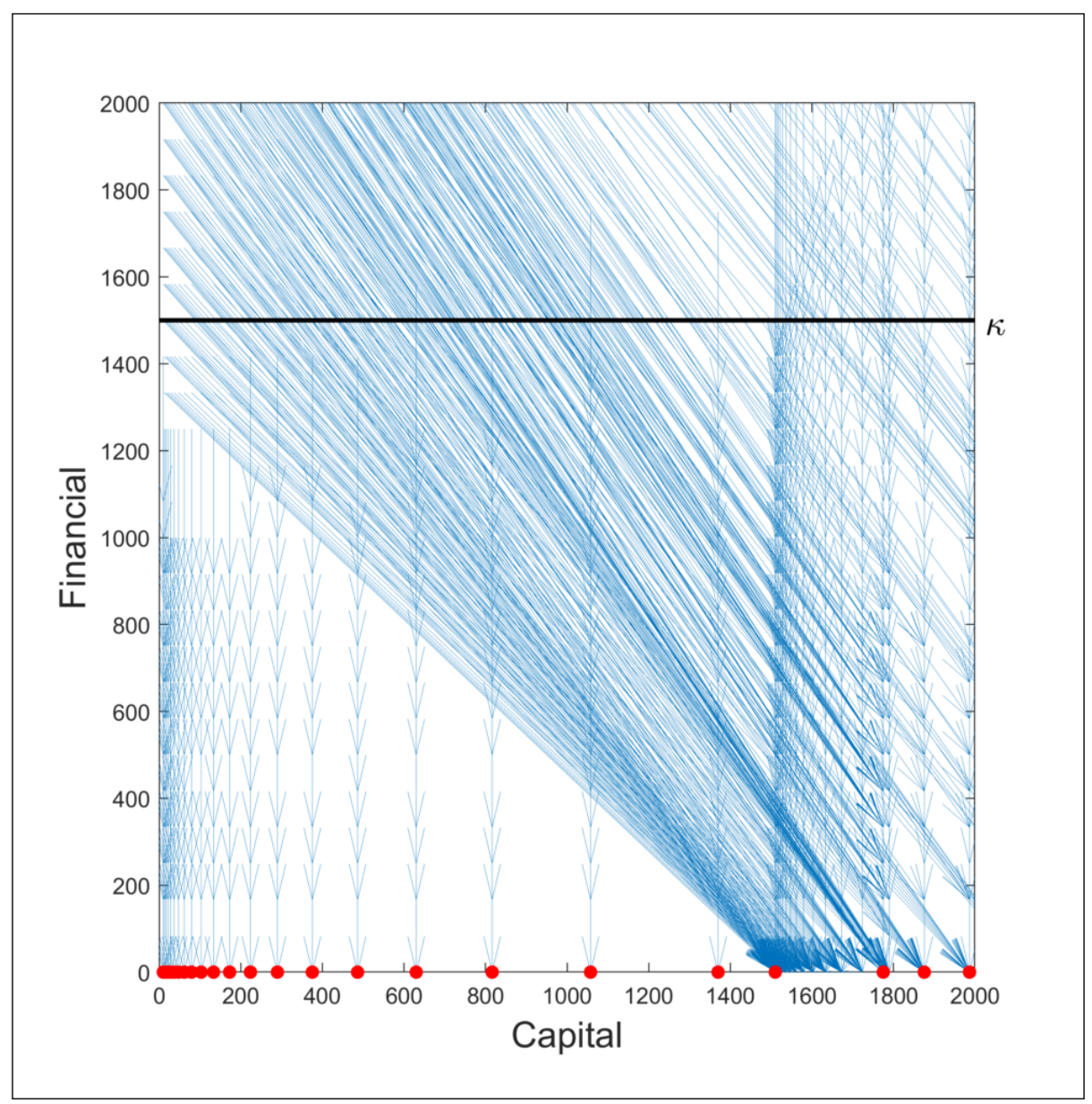




\section{O.7 Belief-elicitation exercise: depreciation and partial irreversibility parameters}

In this section, we describe the procedure used to estimate two parameters that we then fixed in the structural model: depreciation and partial irreversibility. ${ }^{6}$ Following Gavazza, Lizzeri, and Roketskiy (2014), we define irreversibility as the difference between an asset's suggested retail value (the price at which a dealer sells it) and its wholesale price (the price that retailers pay to buy it), holding asset age constant. Table A.50 provides an overview of the variables and parameters used in this estimation procedure.

Table A.50: Overview of variables and parameters

\begin{tabular}{lll}
\hline Variable & Description & Method \\
\hline$\tilde{P}_{t}^{r}$ & Belief about current retail value & Directly collected \\
$\tilde{P}_{t}^{w}$ & Belief about current (wholesale) market value & Directly collected \\
$\tilde{P}_{0}^{r}$ & Original purchase price & Directly collected, reflated using $\tilde{\pi}$ \\
$\tilde{P}_{0}^{w}$ & Theoretical value & Jointly implied by $\tilde{P}_{0}^{r}, \tilde{P}_{t}^{w}, \tilde{\alpha}_{0}$, and $\tilde{\delta}^{w}$ \\
\hline Parameter & Interpretation & Estimation \\
\hline$\tilde{\pi}$ & Inflation estimate & Directly collected \\
$\tilde{\alpha}_{t}$ & Current partial irreversibility rate & $\frac{\tilde{P}_{t}^{r}-\tilde{P}_{t}^{w}}{\tilde{P}_{t}^{r}}$ \\
$\tilde{\delta}^{r}$ & Depreciation rate of retail price & $1-\left(\frac{\tilde{P}_{t}^{r}}{\tilde{P}_{0}^{r}}\right)$ \\
$\tilde{\alpha}_{0}$ & Immediate depreciation at purchase & $\frac{\tilde{P}_{t}^{r}-\tilde{P}_{t}^{w}}{\tilde{P}_{0}^{r}}$ \\
$\tilde{\delta}^{w}$ & Depreciation rate of wholesale price & $1-\left(\frac{\tilde{P}_{t}^{w}}{\left(1-\tilde{\alpha}_{0}\right) \cdot \tilde{P}_{0}^{r}}\right)^{\frac{1}{t}}$ \\
\hline
\end{tabular}

\section{O.7.1 Estimation procedure}

We gathered incentivised beliefs about assets' current wholesale market value $\tilde{P}_{t}^{w}$, and non-incentivised beliefs about the higher retail price $\tilde{P}_{t}^{r}$ charged by dealers. Respondents also stated their recollection of the original purchase price $\tilde{P}_{0}^{r}$, reflated using their inflation estimate $\tilde{\pi}$. These three variable allow the direct computation of $\tilde{\alpha}_{t}=1-\frac{\tilde{P}_{t}^{w}}{\tilde{P}_{t}^{r}}$ and $\tilde{\delta}^{r}=1-\left(\frac{\tilde{P}_{t}^{r}}{\tilde{P}_{0}^{r}}\right)^{\frac{1}{t}}$. The variables of interest $\tilde{\alpha}_{0}$ and $\tilde{\delta}^{w}$, required to calculate the wholesale depreciation schedule, are then estimated as follows:

\footnotetext{
${ }^{6}$ The data and analysis in this section is based on the work of Saidani (2020), who conducted all of the belief elicitation activities with our respondents.
} 
(i) We make use of the fact that most assets are relatively new, varying between 1.7 and 2.9 years, with a mean of 2.3. With the assumption of a near-constant absolute cost of irreversibility being both well-founded theoretically and confirmed empirically, we proceed by applying $\tilde{P}_{0}^{r}-\tilde{P}_{0}^{w} \approx \tilde{P}_{t}^{r}-\tilde{P}_{t}^{w}$ to calculate $\tilde{\alpha}_{0}$ as $\frac{\tilde{P}_{t}^{r}-\tilde{P}_{t}^{w w}}{\tilde{P}_{0}^{r}}$ (the presentday dealer markup divided by the reflated original purchase price).

(ii) Then, noting that $\tilde{P}_{t}^{w}=\tilde{P}_{0}^{w} \cdot\left(1-\tilde{\delta}^{w}\right)^{t}=\tilde{P}_{0}^{r} \cdot \tilde{\alpha}_{0} \cdot\left(1-\tilde{\delta}^{w}\right)^{t}$, it is straightforward to calculate $\tilde{\delta}^{w}=1-\left(\frac{\tilde{P}_{t}^{w}}{\left(1-\tilde{\alpha}_{0}\right) \cdot \tilde{P}_{0}^{r}}\right)^{\frac{1}{t}}$.

\section{O.7.2 Sample and survey}

The sample frame consisted of 193 microenterprise owners from the experimental sample, covering the five largest asset categories for assets purchased in the project. Entrepreneurs received $\$ 3$ for participating in an initial survey, plus up to $\$ 3$ for a followup valuation exercise. Of the 193 individuals in the sample, 130 completed the survey. The reasons for non-completion are as follows: By the time surveys were conducted (October until December 2019), 19\% of the entrepreneurs in the sample had successfully completed the 18-month contract with the MFI, gained full ownership of the asset, and decided to sell it on the market, thereby excluding them from a valuation exercise that required physical ownership of an asset. A further $12 \%$ of respondents were not contactable, partly because they had changed their phone number. Lastly, two respondents refused to be interviewed, in one case due to a health emergency in the immediate family; one individual had moved to a different town; and one person had passed away.

Among others, the survey included the following questions:

Purchase price $\tilde{P}_{0}^{r}$ : What was the purchase price of the asset?

Inflation estimate $\tilde{\pi}$ : "We are interested in the inflation of asset prices over the last years. If you bought the same asset again today in the original condition in which it was purchased, how much would you have to pay for it?".

As a follow-up, respondents were presented with the implied inflation rate, calculated as $\frac{\text { purchase price }}{\text { reflated price }}{ }^{36 / \text { (today-purchase date) }}-1$ : “Your answer implies that a asset changes in value by $\mathrm{x} \%$ each year. Would you like to confirm your estimate, or correct it?"

Market value $\tilde{P}_{t}^{w}$ : Respondents' beliefs about the market value of their assets, the key result of this survey, was elicited in an incentivised manner, as discussed below. The question was worded as follows: "Please think about how much your asset would sell for in its current condition in the market, after negotiating and finding the best price. Think carefully, and consider all important factors. We will later obtain a professional estimate by asset vendors, and if your estimate is within $15 \%$ of the average valuation, you will receive the agreed payment amount. How much would your asset sell for in its current condition in the market?" 
Repurchase value $\tilde{P}_{t}^{r}$ : "Now rather than selling, imagine you had to buy your asset in its current condition from the market. As vendors make a profit on buying and selling, you would probably not be able to buy it for the sale price you mentioned earlier. Please think of the lowest price that a dealer would sell your asset for, after negotiation. How much would you have to pay for your asset if you had to buy it in its current condition from the market?"

\section{O.7.3 Incentivisation}

Respondents were due to be paid a fixed sum if their response was within $15 \%$ of the average valuation of three professional asset valuers. ${ }^{7}$ Given this method, respondents faced the following optimisation problem:

$$
\max _{\tilde{I}} \sum_{\omega} q_{\omega} \mathbb{I}\{0.85 \cdot \bar{\omega} \leq \tilde{\omega} \leq 1.15 \cdot \bar{\omega}\} \cdot u(x)
$$

where $\bar{\omega}=\frac{1}{3} \sum_{i=1}^{3} \omega_{i}$ is the average of three valuations; and $\mathbb{I}\{$ cond $\}$ is an indicator function that equals 1 if the condition is true and 0 otherwise. This method effectively elicits the midpoint of the 'most likely interval' of width $0.3 \cdot \mathrm{E}(\bar{\omega})$, i.e. that interval which includes the most probability density Schlag and van der Weele (2015). ${ }^{8}$ This method retains its incentive-compatibility when agents are not subjective expected utility maximisers: A preference for positive payment over no payment is all that is required (Schlag, Tremewan, and van der Weele, 2015).

Addressing two potential objections, there are two observations worth noting:

- First, respondents' beliefs about the distribution of expert valuations is equivalent to their belief about the distribution of market values, and that it is thus valid to incentivise the latter through the realised value of the former: Experts are simply asset vendors (i.e. those people who buy and sell second-hand assets), and thus their (truthful) assessment of an asset's post-negotiation market value is identical to the price that its owner could expect it to fetch in the market.

- Second, the validity of the incentivisation is not affected by the fact that it was not possible to obtain expert valuations after all, as mentioned previously. Ex-ante, entrepreneurs responded to the survey in the belief that they could receive monetary incentives; and they were not misled, since the valuation exercise was intended and planned, but as mentioned it was ultimately disrupted by the outbreak of Covid-19 and subsequent lockdown in Lahore.

\footnotetext{
${ }^{7}$ Unfortunately, this valuation were not completed as the exercise coincided with the outbreak of Covid19 and the lockdown in Lahore.

${ }^{8}$ In theory, this is a generalisation of the mode: The 'most likely interval' of width 0 is equivalent to the point with the highest probability density, i.e. the mode of the distribution. Nonetheless, except for highly skewed or multi-modal distributions, this quantity is expected to lie close to the mean and the median. Evidence from interviews during the survey trials indicates that respondents' beliefs are single-peaked, and that their uncertainty around stated beliefs is near-symmetric, implying that the mode, mean and median are practically equivalent.
} 


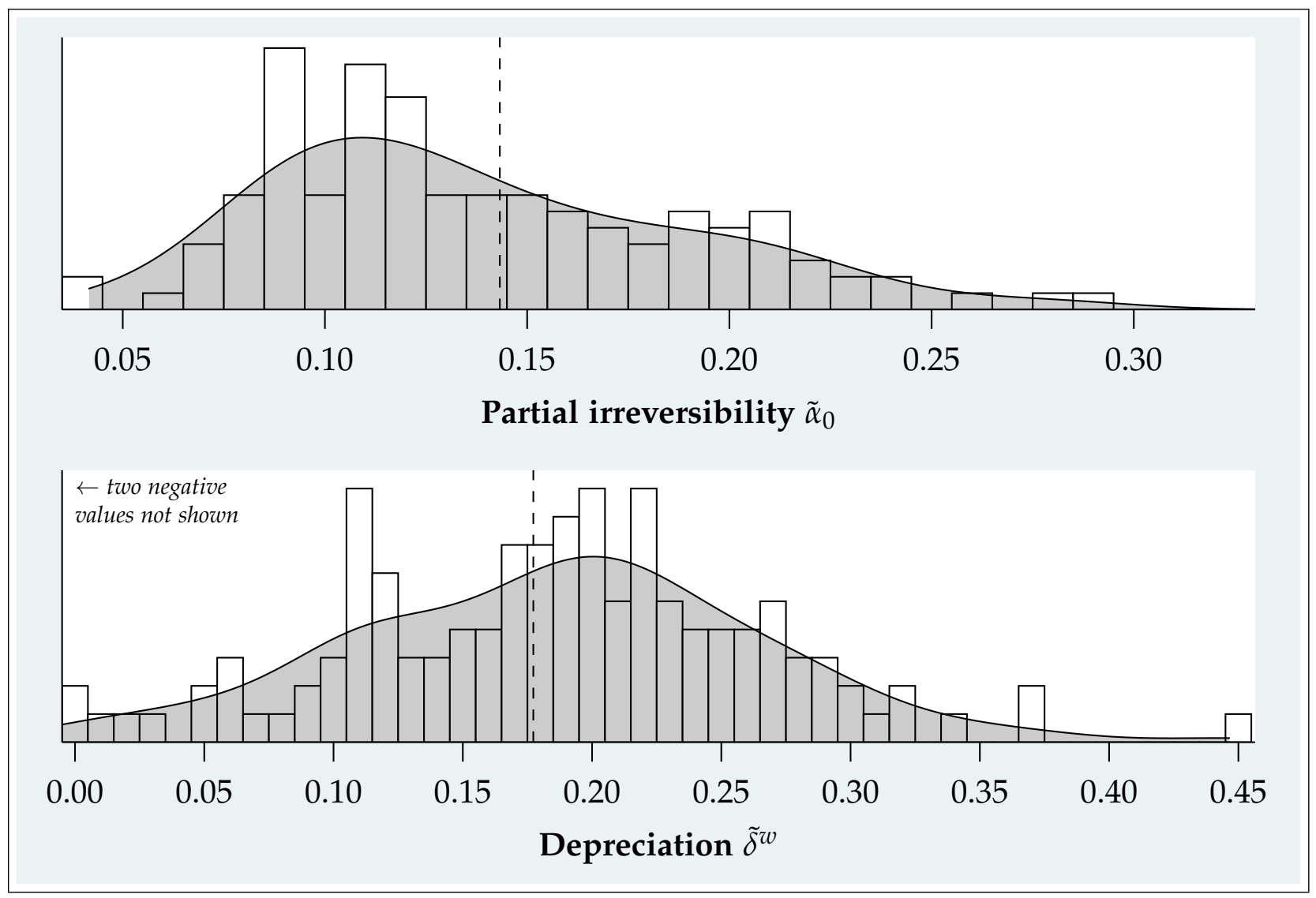

Figure A.13: Density plots of estimated parameters. Dashed lines indicate the sample mean.

\section{O.7.4 Results}

Following the methodology discussed above, we use the data from the microenterprise survey to compute partial irreversibilities $\tilde{\alpha}_{0}$ and exponential depreciation rates $\tilde{\delta}^{w}$ for all microenterprises, which together fully specify the wholesale depreciation schedule:

$$
\begin{aligned}
& \tilde{\alpha}_{0}=\frac{\tilde{P}_{t}^{r}-\tilde{P}_{t}^{w}}{\tilde{P}_{0}^{r}} \\
& \tilde{\delta}^{w}=1-\left(\frac{\tilde{P}_{t}^{w}}{\left(1-\tilde{\alpha}_{0}\right) \cdot \tilde{P}_{0}^{r}}\right)^{\frac{1}{t}}
\end{aligned}
$$

Figure A.13 shows how the estimated parameters are distributed. There is substantial heterogeneity between microenterprises, with implied rates of partial irreversibility ranging from 0.04 to 0.29 , and subjective depreciation even more spread out. The sample averages of $\tilde{\alpha}_{0}$ and $\tilde{\delta}^{w}$ are $14.3 \%$ and $17.7 \%$ respectively. Subjective depreciation schedules implied by these parameters are plotted in A.14. 


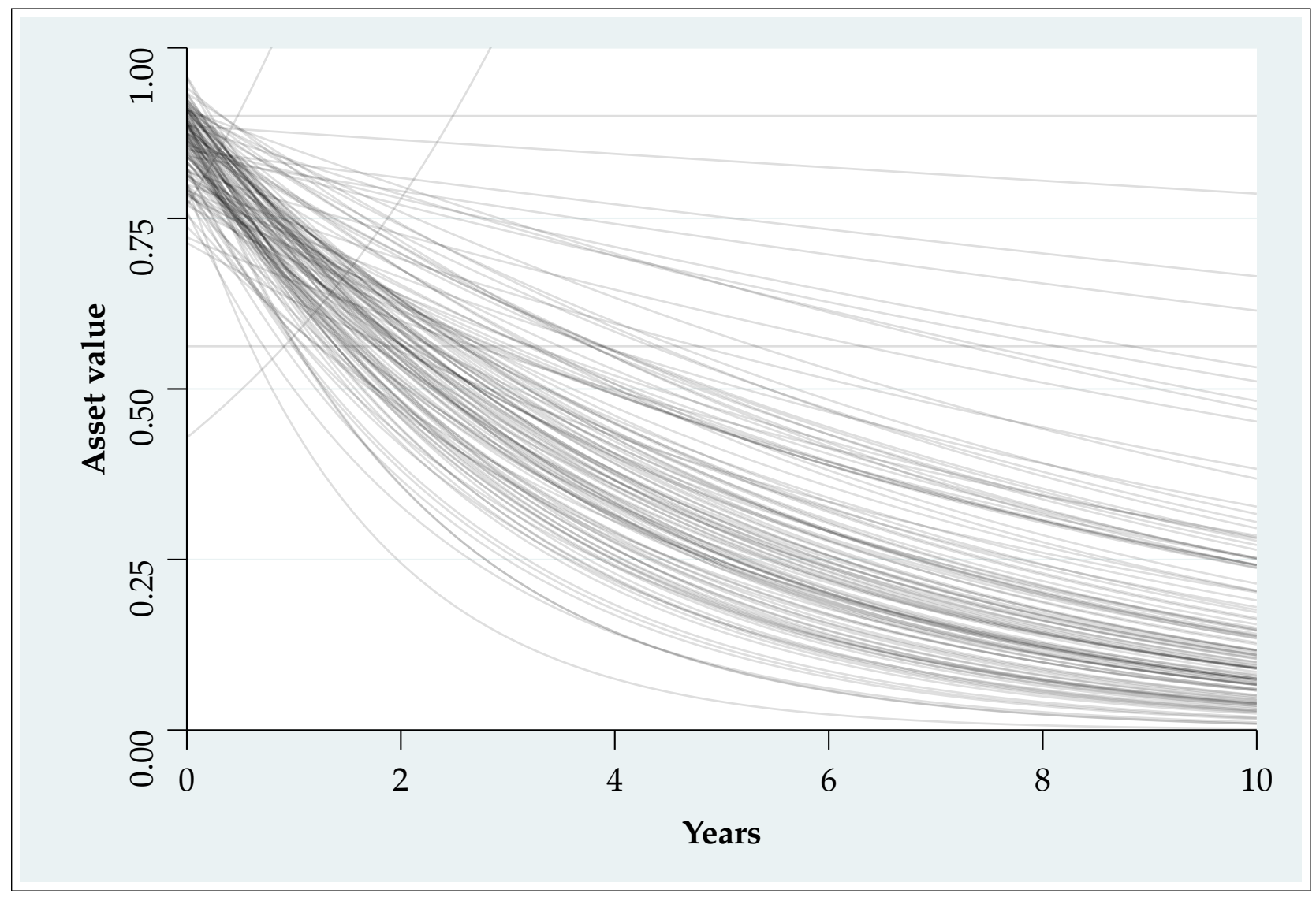

Figure A.14: Subjective depreciation schedules of surveyed microenterprises 


\section{References}

Acharya, A., M. Blackwell, and M. Sen (2016). Explaining Causal Findings Without Bias: Detecting and Assessing Direct Effects. American Political Science Review 110(3), 512-529.

Athey, S. and G. W. Imbens (2017). The Econometrics of Randomized Experiments. In Handbook of Economic Field Experiments, Volume 1, pp. 73-140. Elsevier.

Barr, A. and T. G. Packard (2002). Revealed Preference and Self-insurance: Can we learn from the Self-employed in Chile? Working paper.

Bartling, B., E. Fehr, and H. Herz (2014). The Intrinsic Value of Decision Rights. Econometrica 82(6), 2005-2039.

Benjamini, Y., A. M. Krieger, and D. Yekutieli (2006). Adaptive Linear Step-up Procedures that Control the False Discovery Rate. Biometrika 93(3), 491-507.

Blundell, R. and S. Bond (2000). GMM Estimation with Persistent Panel Data: An Application to Production Functions. Econometric Reviews 19(3), 321-340.

Blundell, R., M. Costa Dias, C. Meghir, and J. Shaw (2016). Female Labor Supply, Human Capital, and Welfare Reform. Econometrica 84(5), 1705-1753.

Carroll, C. (2020). Solution Methods for Microeconomic Dynamic Stochastic Optimization Problems. http://www.econ2. jhu.edu/people/ccarroll/solvingmicrodsops.pdf.

Charness, G., U. Gneezy, and B. Halladay (2016). Experimental Methods: Pay One or Pay All. Journal of Economic Behavior \& Organization 131, 141-150.

Gavazza, A., A. Lizzeri, and N. Roketskiy (2014). A Quantitative Analysis of the Used-Car Market. American Economic Review.

Gelman, A. and D. K. Park (2009). Splitting a Predictor at the Upper Quarter or Third and the Lower Quarter or Third. The American Statistician 63(1), 1-8.

Levinsohn, J. and A. Petrin (2003). Estimating production functions using inputs to control for unobservables. The Review of Economic Studies 70(2), 317-341.

Saidani, Y. (2020). Asset depreciation, partial irreversibility and microenterprise investment decisions in Pakistan. Oxford MPhil thesis.

Schlag, K. H., J. Tremewan, and J. J. van der Weele (2015). A Penny for your Thoughts: A Survey of Methods for Eliciting Beliefs. Experimental Economics.

Schlag, K. H. and J. J. van der Weele (2015). A method to elicit beliefs as most likely intervals. Judgment and Decision Making.

Tauchen, G. (1986). Finite State Markov-chain Approximations to Univariate and Vector Autoregressions. Economics letters 20(2), 177-181.

Vieider, F. M., M. Lefebvre, R. Bouchouicha, T. Chmura, R. Hakimov, M. Krawczyk, and P. Martinsson (2015). Common Components of Risk and Uncertainty Attitudes Across Contexts and Domains: Evidence from 30 Countries. Journal of the European Economic Association 13(3), 421-452. 